\title{
Erdős-Gyárfás Conjecture for Cubic Planar Graphs
}

\author{
Christopher Carl Heckman \\ School of Mathematical and Statistical Sciences \\ Arizona State University \\ Tempe, AZ 85287 - 1804 \\ checkman@math.asu.edu
}

\author{
Roi Krakovski \\ Department of Computer Science \\ Ben-Gurion University \\ Beer-Sheva, 84105, Israel \\ roikr@math.bgu.ac.il
}

Submitted: May 23, 2011; Accepted: Mar 30, 2013; Published: Apr 9, 2013

\begin{abstract}
In 1995, Paul Erdős and András Gyárfás conjectured that for every graph of minimum degree at least 3 , there exists a non-negative integer $m$ such that $G$ contains a simple cycle of length $2^{m}$. In this paper, we prove that the conjecture holds for 3-connected cubic planar graphs. The proof is long, computer-based in parts, and employs the Discharging Method in a novel way.
\end{abstract}

Keywords: Erdős-Gyárfás Conjecture, Cycles of prescribed lengths, Cubic graphs.

\section{Introduction}

In this paper all graphs are finite and simple. Paths and cycles are simple, that is, have no "repeated" vertices. A $k$-cycle is a cycle of length $k$. The well-known Erdős-Gyárfás conjecture [1] states that every graph of minimum degree at least 3 contains a $2^{m}$-cycle, for some $m \geqslant 2$.

A graph is planar if it can be embedded in the plane without crossing edges. A plane graph is an embedded planar graph. A graph $G$ is 3-connected if $|V(G)| \geqslant 4$ and there is no $S \subseteq V(G)$ such that $|S|<3$ and $G \backslash S$ is disconnected ( $\backslash$ denotes deletion). A graph $G$ is cubic if every vertex of $G$ is of degree three.

By computer searches, Markström [2] verified the conjecture for cubic graphs of order at most 29, and found that the smallest cubic planar graph with no 4- or 8-cycles has 24 vertices (see Figure 1). Note that this graph contains a 16-cycle. Shauger [3] proved the conjecture for $K_{1, m}$-free graphs of minimum degree at least $m+1$ or maximum degree at least $2 m-1$. Daniel and Shauger [4] proved the conjecture for planar claw-free graphs. The following is the main result of this paper.

1.1. Every 3-connected cubic planar graph contains a $2^{m}$-cycle, for some $2 \leqslant m \leqslant 7$.

It is not clear whether 1.1 is tight. It is possible that $2 \leqslant m \leqslant 7$ in 1.1 can be replaced with $2 \leqslant m \leqslant 4$. The proof of 1.1 implies the following corollary (which implies a linear time algorithm for detecting a $2^{m}$-cycle): 


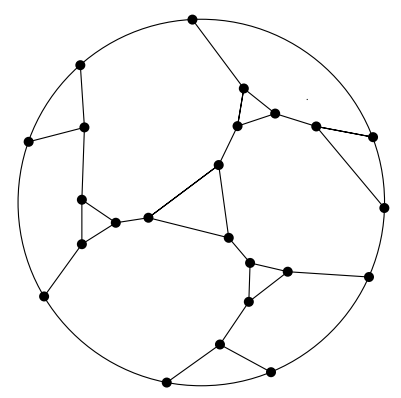

Figure 1: A 3-connected cubic planar graph, with no 4- or 8-cycles.

1.2. There exists an absolute constant, $c$, such that every 3-connected cubic plane graph $G$ has a face $f \in F(G)$ with $|f| \leqslant 71$ and a subgraph $H \subseteq G$ with $|V(H)| \leqslant c$ such that the following holds:

1. $f \subseteq H$ and for every $v \in V(H)$ there exists $u \in V(f)$ and a path of length at most six between $v$ and $u$ in $H$.

2. $H$ contains a $2^{m}$-cycle, for some $2 \leqslant m \leqslant 7$.

We say that two cycles in a graph intersect if they have at least one vertex in common. Thus, if two cycles in a cubic graph intersect, then they have at least one edge in common.

It is well-known that two distinct faces in a 3-connected plane graph have at most one edge in common (or equivalently, the dual graph of a 3-connected plane graph is simple). As this fact is used frequently, it is stated in the following lemma.

1.3. Let $G$ be a 3-connected cubic plane graph, and let $f_{1}, f_{2} \in F(G)$ be distinct. Then either $f_{1}$ and $f_{2}$ are disjoint, or $V\left(f_{1}\right) \cap V\left(f_{2}\right)=\{u, v\}$ and $u v \in E(G)$.

For a graph $G$, we denote by $G \backslash X$ the graph obtained by deleting $X$, where $X$ can be a vertex or an edge, or a set of vertices or edges. For a set $X \subseteq V(G)$, we denote by $G[X]$ the subgraph of $G$ induced by the vertices of $X$. Similarly, for a set $X \subseteq E(G)$, $G[X]$ is the subgraph of $G$ induced by the edges of $X$.

For subgraphs $A_{1}, A_{2} \subseteq G$, disjoint means vertex-disjoint. By $A_{1} \cup A_{2}$ we mean the subgraph of $H$ with vertex-set $V\left(A_{1}\right) \cup V\left(A_{2}\right)$ and edge-set $E\left(A_{1}\right) \cup E\left(A_{2}\right)$.

Let $P$ be a path (we consider paths as subgraphs). It is said to be an $(s, t)$-path if its ends are $s$ and $t$. The length of $P$, denoted $|P|$, is its number of vertices (note the unusual notation). If $P=\emptyset$, then $|P|=0$; otherwise $s \neq t$ or $|P|=1$. Let $S \subseteq G$. We say that $P$ is internally-disjoint from $S$, if $P$ and $S$ are disjoint except possibly for the endpoints of $P$.

Let $H$ be a 2-connected plane graph. The set of vertices, edges and faces of $H$ are denoted by $V(H), E(H)$ and $F(H)$, respectively. A vertex $v \in V(H)$ is a $k$-vertex if its degree is $k$. Similarly, a face $f \in F(H)$ is a $k$-face if $|V(f)|=k$, and then cardinality $k$ is denoted as $|f|$. We write $\leqslant k(\geqslant k)$ for integers smaller or equal (greater or equal) to $k$.

For a $k$-vertex $v \in V(H)$ we denote by $\Gamma_{H}(v)$ the set of faces incident with $v$. A vertex $v$ is a $\left(a_{1}, \ldots, a_{k}\right)$-vertex, if it is a $k$-vertex and the faces incident with $v$ have size (in either a clockwise or an anti-clockwise order around $v$ ) $a_{1}, \ldots, a_{k}$.

For a face $f \in F(H)$, we denote by $\Gamma_{H}(f)$ the set of faces adjacent to $f$. A face $f$ is a $\left(a_{1}, \ldots, a_{k}\right)$-face, if it is a $k$-face and the faces adjacent to $f$ have size (in either a clockwise or an anti-clockwise order around $v) a_{1}, \ldots, a_{k}$. 
If $C$ is a cycle in a plane graph then $\operatorname{int}(C)(\operatorname{ext}(C))$ is the set of vertices and edges inside (outside) $C$ but not on $C$.

Sketch of proof. We prove 1.1 by a way of contradiction. Suppose that the theorem is false and let $G$ be a counterexample. We start by defining a set $\Im_{\text {all }}$ of graphs having some special properties. Then for every $f \in F(G)$ we define a set of subgraphs of $G, \Pi_{f}$, as follows:

1. Every member of $\Pi_{f}$ is isomorphic to some member of $\Im_{\text {all }}$.

2. The members of $\Pi_{f}$ are "almost" pairwise disjoint.

3. Every member of $\Pi_{f}$ has at least one edge in common with $f$.

4. Subject to (1), (2) and (3), $\left|\Pi_{f}\right|$ is maximal.

For each $X \in \Im_{\text {all }}$, let $S_{X}$ be the number of graphs in $\Pi_{f}$ isomorphic to $X$. Then, by the assumption that $G$ contains no $2^{m}$-cycles $(m=2, \ldots, 7)$, we show that for every $X \in \Im_{\text {all }}$ there is a constant $c_{X}$ such that $\sum_{X \in \Im_{\text {all }}} S_{X} \cdot c_{X} \leqslant \varphi(|f|)$. (Where $\varphi: \mathbb{N} \rightarrow \mathbb{N}$ is a predefined function and $\mathbb{N}$ is the set of positive integers.) Then, using the Discharging Method, we show that there is a face $f \in F(G)$ for which $\sum_{X \in \Im_{\text {all }}} S_{X} \cdot c_{X}>\varphi(|f|)$, thus obtaining a contradiction to the existence of $G$.

Organization. In Section 2 we study the intersection between sets of faces of relatively small lengths in a counterexample, $G$. In Section 3 the set $\Im_{\text {all }}$ is defined, and for every $f \in F(G)$, the set $\Pi_{f}$ is constructed. In Section 4, we show how the members of $\Pi_{f}$ are used to construct cycles of prescribed lengths. Finally, in Section 5, the main theorem is proved using the discharging method. This is done by formulating and solving a set of integer linear programs.

\section{Basic Properties of a Counterexample}

Throughout $G$ denotes a counterexample to 1.1 , that is,

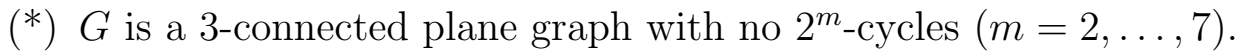

The following topological lemma will be useful.

2.1. Let $C$ be a cycle of $G$ and let $v_{1}, u_{1}, v_{2}$ and $u_{2}$ be four vertices of $C$ appearing in order in a clockwise (or anti-clockwise) traversal of $C$ starting from $v_{1}$. Then there do not exist two disjoint paths $P$ and $Q$, internally disjoint from $C$, such that $P$ is a $\left(v_{1}, v_{2}\right)$-path, $Q$ is a $\left(u_{1}, u_{2}\right)$-path, and $V(P), V(Q) \subseteq V(f)$ for some $f \in F(G)$.

The following lemma is straightforward.

2.2. For $m=2, \ldots, 7$, the following holds in $G$ :

1. Let $f_{1}, \ldots, f_{k} \in F(G)$ be distinct such that the subgraph of the dual graph of $G$, induced by the vertices corresponding to $f_{1}, \ldots, f_{k}$ is a tree (i.e, a connected acyclic graph). Then $\sum_{i=1}^{k}\left|f_{i}\right| \neq 2(k-1)+2^{m}$. 
2. If $v \in V(G)$ be $a(p, q, r)$-vertex $(p, q, r \in \mathbb{N})$, then $p+q+r \neq 6+2^{m}$.

3. Let $f_{1}, f_{2} \in F(G)$ be adjacent. Let $g_{1}, g_{2}, g_{3} \in F(G) \backslash\left\{f_{1}, f_{2}\right\}$ be distinct 3-faces, and suppose that for $i=1,2, g_{i}$ is adjacent to $f_{1}$ and $f_{2}$. Then $g_{3}$ is disjoint from at least one of $f_{1}$ and $f_{2}$.

The following corollary will be used frequently.

2.3. The following holds for $G$ :

(0) there are no 4-, 8-or 16-cycles.

(1) no two 3-faces are adjacent.

(2) no two 5-faces are adjacent.

(3) a 6-face is adjacent to at most one 3-face.

(4) $G$ contains no $(3,5,6)$-vertex.

(5) no two 9-faces are adjacent.

(6) $G$ contains no $(9,3,10)$-vertex.

\subsection{Properties of a 3-face not adjacent to 5-faces}

Let $f \in F(G)$ be a 3 -face in $G$. Let $x_{1}, x_{2}, x_{3}$ be the vertices of $f$ in a cyclic clockwise ordering. Let $y_{i}(i=1,2,3)$ be the neighbor of $x_{i}$ other than $x_{i-1}$ and $x_{i+1}$. Set $x_{4}:=x_{1}$, $x_{0}:=x_{3}, y_{4}:=y_{1}$ and $y_{0}:=y_{3}$. As $G$ is simple, $y_{i} \notin V(f)$. By 2.3(0), for distinct $1 \leqslant i, j \leqslant 3, y_{i} \neq y_{j}$. Let $f_{i} \in F(G) \backslash\{f\}(i=1,2,3)$ such that $f_{i}$ is incident to $x_{i} x_{i+1}$. Suppose $f_{i}=x_{i} y_{i} p_{1}^{i} p_{2}^{i} \ldots p_{\left|f_{i}\right|-4}^{i} y_{i+1} x_{i+1}$ and let $P^{i}=p_{1}^{i} p_{2}^{i} \ldots p_{\left|f_{i}\right|-4}^{i}$.

By 1.3, $V\left(f_{i}\right) \cap V\left(f_{i+1}\right)=\left\{x_{i+1}, y_{i+1}\right\}$, and hence for distinct $1 \leqslant i, j \leqslant 3, P^{i}$ and $P^{i+1}$ are disjoint. Also by $2.3(0,1),\left|P^{i}\right| \geqslant 1$, for $i=1,2,3$. Let

$$
S=f_{1} \cup f_{2} \cup f_{3}
$$

Two disjoint faces $f^{\prime}, f^{\prime \prime} \in F(G)$ are called semi-adjacent if there exist $v \in V\left(f^{\prime}\right)$ and $u \in V\left(f^{\prime \prime}\right)$ such that $u v \in E(G)$. Note that cubicity of $G$ implies that a $k$-face has at most $k$ semi-adjacent faces. For $i=1,2,3$, let $g_{i}$ be the face incident with $y_{i}$, other than $f_{i-1}$ and $f_{i}$. Then $g_{1}, g_{2}, g_{3}$ are the three semi-adjacent faces of $f$.

\subsubsection{Properties of a 3 -face adjacent to three 6 -faces}

Assume that $\left|f_{i}\right|=6$, for $i=1,2,3$. The following claim follows merely from 2.3(0).

2.4. Let $1 \leqslant i \leqslant 3,1 \leqslant j \leqslant 2$ and let $Q$ be $a\left(p_{j}^{i}, p_{j}^{i+1}\right)$-path internally disjoint from $S$ (where $p_{j}^{4}:=p_{j}^{1}$ ). Then $|Q|=5$ or $|Q| \geqslant 13$.

2.5. Let $f^{*} \in F(G) \backslash\left\{f, f_{1}, f_{2}, f_{3}\right\}$ and suppose $V\left(f^{*}\right) \cap V(S) \neq \emptyset$.

1. If $f^{*}=g_{i}$ (for some $i \leqslant i \leqslant 3$ ) and $\left|f^{*}\right| \leqslant 18$, then $\left|V(S) \cap V\left(g_{i}\right)\right|=3$.

2. If $\left|f^{*}\right| \leqslant 9$ and $f^{*} \neq g_{i}$ (for some $i=1,2,3$ ), then $\left|V(S) \cap V\left(f^{*}\right)\right|=2$. 
Proof. (1) Without loss of generality assume that $f^{*}=g_{1}$. Let $k=\left|V\left(g_{1}\right)\right|-3$. Put $q_{0}:=p_{2}^{3}, q_{k+1}:=p_{1}^{1}$ and $q_{k+2}=y_{1}$. Let $Q:=q_{1} \ldots q_{k}$ be a path on $g_{1}$ such that for $0 \leqslant i \leqslant k, q_{i} q_{i+1} \in E(f)$. As $q_{0}, q_{k+1}, q_{k+2} \in V\left(g_{1}\right) \cap S$, we have to show that $Q$ and $S$ are disjoint.

Suppose to the contrary that $Q$ and $S$ are not disjoint. By $1.3, y_{2}, y_{3}, p_{2}^{1}, p_{1}^{3} \notin V(Q) \cap$ $V(S)$. As $G$ is cubic, $\left|V\left(g_{1}\right) \cap S\right|=5$ and $V(Q) \cap V(S)=\left\{p_{1}^{2}, p_{2}^{2}\right\}$. Let $1 \leqslant j \leqslant k$ such that $q_{j} \in V(S)$, and if $k \geqslant 2$, then $q_{1}, \ldots, q_{j-1} \notin V(S)$. Let $Q_{1}=q_{0} q_{1} \ldots q_{j-1} q_{j}$ and $Q_{2}=q_{j+1} \ldots q_{k+1}$. By definition, $Q_{1}$ and $Q_{2}$ are disjoint and each is internally-disjoint from $S$. By $2.1, Q_{1}$ is a $\left(q_{0}, p_{2}^{2}\right)$-path, and $Q_{2}$ is a $\left(q_{k+1}, p_{1}^{2}\right)$-path. As $\left|g_{1}\right| \leqslant 18$ and $\left|V\left(g_{1}\right) \cap S\right|=5$, we see that $\left|Q_{1}\right|+\left|Q_{2}\right|=\left|g_{1}\right|-1 \leqslant 17$. By 2.4, it must be that $\left|Q_{1}\right|=\left|Q_{2}\right|=5$. But then $S \cup Q_{1} \cup Q_{2} \subseteq G$ contains a 16-cycle; a contradiction.

(2) For $i=1,2,3$, let $h_{i}$ denote the face adjacent to $f_{i}$ so that $E\left(f_{i}\right) \cap E\left(h_{i}\right)=\left\{\begin{array}{c}p_{1}^{i} \\ p_{2}^{i}\end{array}\right\}$ and suppose that $h_{i} \neq\left\{g_{1}, g_{2}, g_{3}\right\}$. Then $\left|E(S) \cap E\left(h_{i}\right)\right| \geqslant 1$ and $E\left(h_{i}\right) \cap E(S) \subseteq \bigcup_{i=1}^{3}\left\{p_{1}^{i} p_{2}^{i}\right\}$. Now suppose to the contrary that for some $1 \leqslant i \leqslant 3$ there exist $1 \leqslant j \neq i \leqslant 3$ so that $p_{1}^{j} p_{2}^{j} \in E\left(h_{i}\right)$. By symmetry we may assume that $i=1$ and $j=2$. By 2.1 , there is a $\left(p_{2}^{1}, p_{1}^{2}\right)$-path $Q \subseteq h_{1}$ internally disjoint from $S$. If $|Q|=2$ (then $g_{2}$ is a 3 -face) and we get a contradiction to 2.3. If $|Q| \geqslant 3$ then $\left\{p_{2}^{1}, p_{1}^{2}\right\}$ is a 2-cut in $G$ contradicting 3-connectivity of $G$.

2.6. Let $1 \leqslant i, j \leqslant 3$ be distinct. If $5 \leqslant\left|g_{i}\right|,\left|g_{j}\right| \leqslant 7$, then $g_{i}$ and $g_{j}$ are disjoint.

Proof. Suppose not. By symmetry, assume that $i=1, j=2$. By $1.3,\left|V\left(g_{1}\right) \cap V\left(g_{2}\right)\right|=2$.

Let $k:=\left|V\left(g_{1}\right)\right|-3$ and $k^{\prime}:=\left|V\left(g_{2}\right)\right|-3$. Let $Q^{1}:=q_{1} \ldots q_{k} \subseteq g_{1}\left(Q^{2}=u_{1} \ldots u_{k^{\prime}} \subseteq\right.$ $\left.g_{2}\right)$, such that $y_{1} \notin V\left(Q_{1}\right)\left(y_{2} \notin V\left(Q_{2}\right)\right),\left\{q_{1} p_{2}^{3}, q_{k} p_{1}^{1}\right\} \subseteq E\left(g_{1}\right)\left(\left\{u_{1} p_{2}^{1}, u_{k^{\prime}} p_{1}^{2}\right\} \subseteq E\left(g_{2}\right)\right)$, and for $1 \leqslant i \leqslant k-1\left(1 \leqslant i \leqslant k^{\prime}-1\right), q_{i} q_{i+1} \in E\left(g_{1}\right)\left(u_{i} u_{i+1} \in E\left(g_{2}\right)\right)$. By 2.5, $Q_{1}$ and $Q_{2}$ are disjoint from $S$ and since $\left|V\left(g_{1}\right) \cap V\left(g_{2}\right)\right|=2$ then $\left|V\left(Q_{1}\right) \cap V\left(Q_{2}\right)\right|=2$.

There exist $1 \leqslant \ell \leqslant k-1$ and $1 \leqslant \ell^{\prime} \leqslant k^{\prime}-1$ such that $q_{\ell}=u_{\ell^{\prime}+1}$ and $q_{\ell+1}=u_{\ell^{\prime}}$. Then the length of the path $P_{1}=p_{2}^{3}, q_{1}, \ldots, q_{\ell}=u_{\ell^{\prime}+1}, u_{\ell^{\prime}+2}, \ldots, p_{1}^{2}$ is at least 6 (for otherwise there is an 8-cycle in $G$ ). Similarly, the length of the path $P_{2}=p_{2}^{1}, u_{1}, \ldots, u_{\ell^{\prime}}=$ $q_{\ell+1}, q_{\ell+2}, \ldots, p_{1}^{1}$ is at least five. Then $\left|g_{1}\right|+\left|g_{2}\right|=\left|P_{1}\right|+\left|P_{2}\right|+4 \geqslant 15$ (where the +4 comes from the fact that $y_{1}, y_{2} \notin V\left(P_{1} \cup P_{2}\right)$ and that each of $q_{\ell}$ and $q_{\ell^{\prime}}$ is contained in exactly one of $V\left(P_{1}\right)$ and $V\left(P_{2}\right)$ and in both of $V\left(g_{1}\right)$ and $\left.V\left(g_{2}\right)\right)$, but this is a contradiction since $\left|g_{1}\right|,\left|g_{2}\right| \leqslant 7$.

The following is an easy consequence of 2.3(0) and 2.6.

2.7. If $5 \leqslant\left|g_{i}\right| \leqslant 7(1 \leqslant i \leqslant 3)$, then $\left|g_{i-1}\right|,\left|g_{i+1}\right| \geqslant 10$.

2.8. Let $f^{*} \in F(G) \backslash\left\{f, f_{1}, f_{2}, f_{3}\right\}$ and suppose $\left|f^{*}\right| \in\{3,9\}$. Then $f^{*}$ and $S$ are disjoint.

Proof. Suppose not. By 2.3(3) and the assumption that $f^{*} \neq f$, we may assume that $\left|f^{*}\right|=9$. We may also assume that $f^{*} \neq g_{i}$, for $i=1,2,3$. For if $f^{*}=g_{1}$, say, then by 2.5 , $\left|V\left(g_{1}\right) \cap V(S)\right|=3$. Hence, $V\left(g_{1}\right) \cap V(S)=\left\{p_{2}^{3}, y_{1}, p_{1}^{1}\right\}$, and $C:=\left(S \cup f^{*}\right) \backslash\left\{p_{2}^{1}, x_{2}\right\}$ is a 16-cycle, a contradiction.

By $2.5,\left|V\left(f^{*}\right) \cap V(S)\right|=2$, and by symmetry we may assume that $E\left(f^{*}\right) \cap E(S)=$ $\left\{p_{1}^{1} p_{2}^{1}\right\}$. But then $f^{*} \cup f_{1} \cup f_{2} \subseteq G$ contains a 16-cycle; a contradiction.

2.9. Let $f_{1}^{*}, f_{2}^{*} \in F(G) \backslash\left\{f, f_{1}, f_{2}, f_{3}\right\}$ be distinct. Suppose that $\left|f_{1}^{*}\right|,\left|f_{2}^{*}\right| \in\{5,6\}$ and $S \cap\left(f_{1}^{*} \cup f_{2}^{*}\right) \neq \emptyset$. Then 
1. If $5 \in\left\{\left|f_{1}^{*}\right|,\left|f_{2}^{*}\right|\right\}$, then (i) $S$ is disjoint from $f_{1}^{*}$ or $f_{2}^{*}$, and (ii) $f_{1}^{*}$ and $f_{2}^{*}$ are not adjacent.

2. If $\left|f_{1}^{*}\right|,\left|f_{2}^{*}\right|=6$ and $f_{1}^{*}$ and $f_{2}^{*}$ are adjacent, then $S$ is disjoint from $f_{1}^{*}$ or $f_{2}^{*}$.

Proof. (1) First observe that (ii) follows from (i) and the assumption that $G$ contains no 16-cycles. For the proof of (i), we assume for a contradiction that $S \cap f_{1}^{*} \neq \emptyset$ and $S \cap f_{2}^{*} \neq \emptyset$. Without loss of generality, assume that $\left|f_{1}^{*}\right|=5$.

We may assume that $\left|f_{2}^{*}\right|=6$. For if $\left|f_{2}^{*}\right|=5$, then by $2.3(2)$ and by $2.2(1)$, the union of $f_{1}^{*}$ and $f_{2}^{*}$ and two (appropriate) faces from $\left\{f_{1}, f_{2}, f_{3}\right\}$ form a 16-cycle, a contradiction.

Case 1. Suppose $f_{1}^{*}=g_{i}$, for some $1 \leqslant i \leqslant 3$. By symmetry assume that $f_{1}^{*}=g_{1}$. By 2.7, $f_{2}^{*} \notin\left\{g_{2}, g_{3}\right\}$. Since $S \cap f_{2}^{*} \neq \emptyset, E(S) \cap E\left(f_{2}^{*}\right) \subseteq\left\{p_{1}^{1} p_{2}^{1}, p_{1}^{2} p_{2}^{2}, p_{1}^{3} p_{2}^{3}\right\}$. By 2.5, $\left|V(S) \cap V\left(f_{2}^{*}\right)\right|=2$. Hence, there is a unique $1 \leqslant j \leqslant 3$ such that $E(S) \cap E\left(f_{2}^{*}\right)=\left\{p_{1}^{j} p_{2}^{j}\right\}$.

If $j \in\{1,3\}$, say $j=1$, then $f_{1}^{*}$ and $f_{2}^{*}$ are adjacent. But then $\left(S \cup f_{1}^{*} \cup f_{2}^{*}\right) \backslash\left\{x_{3}\right\} \subseteq G$ contains a 16-cycle, a contradiction. Assume $j=2$. Let $f_{2}^{*}:=p_{1}^{2} u_{1} u_{2} u_{3} u_{4} p_{2}^{2}$ and let $Q:=f_{2}^{*} \backslash\left\{p_{1}^{2}, p_{2}^{2}\right\}$. By 2.5, $Q$ and $S$ are disjoint. We may assume that $f_{1}^{*}$ and $f_{2}^{*}$ are not disjoint (and hence $\left|V\left(f_{1}^{*}\right) \cap V\left(f_{2}^{*}\right)\right|=2$ ), for otherwise $\left(S \cup f_{1}^{*} \cup f_{2}^{*}\right) \backslash\left\{p_{1}^{3}, x_{2}\right\} \subseteq G$ is a 16cycle. By 2.1 and since $\left|f_{1}^{*}\right|=5$, we have that $p_{3}^{2} u_{4} \in E(G), p_{3}^{2} u_{3} \in E(G)$ or $p_{3}^{2} u_{2} \in E(G)$. But then we easily see that $S \cup f_{1}^{*} \cup f_{2}^{*} \subseteq G$ contains an 8-cycle; a contradiction.

Case 2. Suppose that $f_{1}^{*} \neq g_{i}$, for $i=1,2,3$. By the same arguments as in Case (1), we conclude that $f_{2}^{*} \neq g_{i}$, for $i=1,2,3$. By symmetry, we may assume that $E\left(f_{1}^{*}\right) \cap$ $E(S)=\left\{p_{1}^{1} p_{2}^{1}\right\}$ and $E\left(f_{2}^{*}\right) \cap E(S)=\left\{p_{1}^{2} p_{2}^{2}\right\}$. Let $f_{1}^{*}:=p_{1}^{1} u_{1} u_{2} u_{3} p_{2}^{1} p_{1}^{1}, Q_{1}:=f_{1}^{*} \backslash\left\{p_{1}^{1}, p_{2}^{1}\right\}$, $f_{2}^{*}:=p_{1}^{2} v_{1} v_{2} v_{3} v_{4} p_{2}^{2} p_{1}^{2}$ and $Q_{2}:=f_{2}^{*} \backslash\left\{p_{1}^{2}, p_{2}^{2}\right\}$. As in Case (1), we see that $f_{1}^{*}$ and $f_{2}^{*}$ must be adjacent. By 2.1, we have that $u_{1} \in\left\{v_{2}, v_{3}, v_{4}\right\}$ or $u_{3} \in\left\{v_{1}, v_{2}, v_{3}\right\}$. But then, in all cases, we can easily find an 8-cycle in $S \cup f_{2}^{*} \subseteq G$; a contradiction.

(2) The proof follows by the similar argument as the proof of (1).

2.10. Suppose $\left|g_{i}\right| \in\{17,18\}$ (for some $1 \leqslant i \leqslant 3$ ). Suppose also that there exist $f^{\prime}, f^{\prime \prime} \in$ $F(G) \backslash\left\{f, f_{1}, f_{2}, f_{3}\right\}$ such that $\left|f^{\prime}\right| \in\{5,6\},\left|f^{\prime \prime}\right|=3, f^{\prime}, f^{\prime \prime}$ and $g_{i}$ are pairwise adjacent. If $\left(S \cap g_{i}\right) \cap\left(f^{\prime} \cup f^{\prime \prime}\right)=\emptyset$, then $S \cap\left(f^{\prime} \cup f^{\prime \prime}\right)=\emptyset$.

Proof. For suppose not. By symmetry assume that $g_{i}=g_{1}$. By $2.5(1), V\left(g_{1}\right) \cap V(S)=$ $\left\{p_{1}^{1}, y_{1}, p_{2}^{3}\right\}$. Let $k:=\left|V\left(g_{1}\right)\right|-3$. Let $Q:=q_{1} \ldots q_{k} \subseteq g_{1}$ such that $y_{1} \notin V\left(Q_{1}\right)$, $\left\{q_{1} p_{2}^{3}, q_{k} p_{1}^{1}\right\} \subseteq E\left(g_{1}\right)$, and for $1 \leqslant i \leqslant k-1, q_{i} q_{i+1} \in E\left(g_{1}\right)$. Let $1 \leqslant r<\ell \leqslant k$ such that $V\left(g_{1}\right) \cap V\left(f^{\prime \prime}\right)=\left\{q_{r}, q_{\ell}\right\}$. Let $v \in V\left(f^{\prime \prime}\right) \backslash\left\{q_{r}, q_{\ell}\right\}$. Clearly, $S \cap f^{\prime \prime}=\emptyset$. As $f^{\prime}$ is adjacent to $g_{1}$ and $f^{\prime \prime}$, then by symmetry, we may assume that $V\left(g_{1}\right) \cap V\left(f^{\prime}\right)=\left\{q_{\ell}, q_{\ell+1}\right\}$.

Hence (as $S \cap f^{\prime \prime}=\emptyset$ but $\left.S \cap\left(f^{\prime} \cup f^{\prime \prime}\right) \neq \emptyset\right), S \cap f^{\prime} \neq \emptyset$. Let $Q_{1}^{*}:=p_{2}^{3} q_{1} \ldots q_{r} \subseteq g_{1}$ and $Q_{2}^{*}:=q_{\ell+1} \ldots q_{k} p_{1}^{1} \subseteq g_{1}$.

Next we show that $f^{\prime} \notin\left\{g_{2}, g_{3}\right\}$. If $f^{\prime}=g_{2}$, then by $2.5, V(S) \cap V\left(f^{\prime}\right)=\left\{p_{1}^{2}, y_{2}, p_{2}^{1}\right\}$. Hence, it can only be that $\left|f^{\prime}\right|=6$. By $2.1, p_{2}^{1} q_{\ell+1} \in E(G)$. Also $\ell+1 \neq k$ (for otherwise $G\left[f \cup f_{1} \cup q_{k}\right]$ contains an 8-cycle). Hence, $\left|Q_{2}^{*}\right| \geqslant 3$; but then $\left\{p_{1}^{1}, p_{\ell+1}\right\}$ is a 2-cut in $G$; a contradiction. Similarly, if $f^{\prime}=g_{3}$, then as above $V(S) \cap V\left(f^{\prime}\right)=\left\{p_{2}^{2}, y_{3}, p_{1}^{3}\right\}$ and $\left|f^{\prime}\right|=6$. By 2.1, $v p_{1}^{3} \in E(G)$. By 2.3(0), $r \neq 1$; but then $\left\{p_{2}^{3}, q_{r}\right\}$ is a 2-cut in $G$; a contradiction.

So assume that $f^{\prime} \notin\left\{g_{2}, g_{3}\right\}$. As $\left(S \cap g_{1}\right) \cap\left(f^{\prime} \cup f^{\prime \prime}\right)=\emptyset$, then $V\left(f^{\prime \prime}\right) \cap V(S)=$ $\left\{p_{1}^{2}, p_{2}^{2}\right\}$. By 2.1, there are disjoint paths $Q_{1}, Q_{2}$, internally-disjoint from $S$, such that $V\left(Q_{1}\right), V\left(Q_{2}\right) \subseteq V\left(f^{\prime}\right), Q_{1}$ is a $\left(v, p_{2}^{2}\right)$-path, and $Q_{2}$ is a $\left(q_{\ell+1}, p_{1}^{2}\right)$-path. As $5 \leqslant\left|f^{\prime}\right| \leqslant 6$ and $\ell \notin V\left(Q_{1} \cup Q_{2}\right),\left|Q_{1}\right|+\left|Q_{2}\right| \leqslant 5$. Hence, $\left|Q_{1}\right|=2$ or $\left|Q_{2}\right|=2$.

We shall assume that $\left|Q_{1}\right|=2$ (as the case that $\left|Q_{2}\right|=2$ and thus $p_{1}^{2} q_{\ell+1} \in E(G)$ follows through similar arguments). Hence we have that $p_{2}^{2} v \in E(G)$ and $2 \leqslant\left|Q_{2}\right| \leqslant 3$. 
Now, using 2.3(0), it is easily verified (even regardless of the exact value of $\left|Q_{2}\right|$ ), that $\left|Q_{2}^{*}\right| \notin\{2, \ldots, 10\}$. As $\left|g_{1}\right| \in\{17,18\}$, and $y_{1}, q_{\ell} \notin V\left(Q_{1}^{*}\right) \cup V\left(Q_{2}^{*}\right)$, then $\left|Q_{1}^{*}\right|+\left|Q_{2}^{*}\right| \leqslant 16$. Hence, we conclude that $2 \leqslant V\left|Q_{1}^{*}\right| \leqslant 6$. But then (for each possible value of $\left|Q_{1}^{*}\right|$ ) it is easy to find an 8- or 16-cycle; a contradiction.

\subsubsection{Properties of a 3-face adjacent to two 6-faces and a 9-face}

Next we assume that $f$ is adjacent to two 6-faces and a 9-face.

2.11. Let $f^{*} \in F(G) \backslash\left\{f, f_{1}, f_{2}, f_{3}\right\}$ such that $\left|f^{*}\right| \in\{5,6,9\}$. Then, $S$ and $f^{*}$ are disjoint, unless $\left|f^{*}\right|=9$ and $f^{*}$ and $f$ are semi-adjacent so that the edge with one end in $V(f)$ and one end in $V\left(f^{*}\right)$ is common to the two 6-faces.

Proof. Suppose not. By symmetry assume that $\left|f_{1}\right|=\left|f_{2}\right|=6$ and $\left|f_{3}\right|=9$.

Case 1. Suppose $f^{*}=g_{i}$, for some $i, 1 \leqslant i \leqslant 3$. Then either $f^{*}=g_{2}$ or $f^{*} \in\left\{g_{1}, g_{3}\right\}$. We shall consider the former case as the latter follows by similar arguments.

Suppose then that $f^{*}=g_{2}$. Let $k:=\left|V\left(g_{2}\right)\right|-3$. Put $q_{0}=p_{2}^{1}, q_{k+1}:=p_{1}^{2}$ and $q_{k+2}:=y_{2}$. Let $Q:=q_{1} q_{2} \ldots q_{k} \subseteq g_{2}$ such that $q_{i} q_{i+1} \in E\left(g_{2}\right)$, for $i=0, \ldots, k$. We may assume that $\left|V(S) \cap V\left(f^{*}\right)\right|>3$. For otherwise, if $\left|f^{*}\right|=9$ the claim follows, and if $\left|f^{*}\right|=\{5,6\}$, then $S \cup f^{*}$ contains a 16-cycle, a contradiction.

By 1.3, we see that $y_{1}, y_{3}, p_{1}^{1}, p_{2}^{2} \notin V(Q) \cap V(S)$ and conclude that $|V(Q) \cap V(S)|=2$. By 2.3(5), we may assume that $\left|g_{2}\right| \in\{5,6\}$. By 2.3(0), neither $p_{2}^{1}$ nor $p_{1}^{2}$ is adjacent to any of $p_{1}^{3}, \ldots, p_{5}^{3}$. Hence, $|V(Q) \cap S|=\left|V(Q) \cap V\left(f_{3}\right)\right| \leqslant 1$; a contradiction.

Case 2. Suppose $f^{*} \neq g_{i}$, for $i=1,2,3$.

Case 2.1 Suppose $V\left(f^{*}\right) \cap V\left(f_{3}\right) \neq \emptyset$. As by Case (1), $f^{*} \neq g_{i}$, for $i=1,2,3$, then $E\left(f^{*}\right) \cap E\left(f_{3}\right)=\left\{p_{r}^{3} p_{\ell}^{3}\right\}$, for some $1 \leqslant r<\ell \leqslant 5$. By 2.3(5), we may assume that $\left|f^{*}\right| \in\{5,6\}$. We see that $f^{*}$ is disjoint from $f_{1}$ or $f_{2}$. For otherwise, by Case (1), $\left\{p_{1}^{1}, p_{2}^{1}, p_{1}^{2}, p_{2}^{2}, p_{r}^{3}, p_{\ell}^{3}\right\} \subseteq V\left(f^{*}\right)$. By $2.2,\left|f^{*}\right| \neq 5$, and if $\left|f^{*}\right|=6$, then by $2.1, p_{2}^{1} p_{1}^{2} \in E(G)$, contradicting $2.3(3)$.

Next we show that $f^{*}$ is disjoint from $f_{1}$ and $f_{2}$. For suppose not. By symmetry, assume that $V\left(f^{*}\right) \cap V\left(f_{1}\right) \neq \emptyset$ and $V\left(f^{*}\right) \cap V\left(f_{2}\right)=\emptyset$. Now, if $\left|f^{*}\right|=5$, then by 2.2(1), $G\left[f_{2} \cup f_{3} \cup f^{*}\right]$ contains a 16-cycle. If $\left|f^{*}\right|=6$, then $G\left[f \cup f_{3} \cup f_{2} \cup f^{*}\right] \backslash\left\{x_{3}\right\}$ is a 16-cycle. Both cases lead to a contradiction. Hence we may assume that $f^{*}$ is disjoint from $f_{1}$ and $f_{2}$. But then as $\left|f^{*}\right| \in\{5,6\}, S \cup f^{*} \subseteq G$ contains a 16-cycle, a contradiction.

Case 2.2 Suppose $V\left(f^{*}\right) \cap V\left(f_{3}\right)=\emptyset$. By Case (1) and 2.2(1), we may assume that $V\left(f^{*}\right) \cap V\left(f_{1}\right) \neq \emptyset$ and $V\left(f^{*}\right) \cap V\left(f_{2}\right) \neq \emptyset$. Let $k:=V\left(f^{*}\right)-4$. By Case (1), and 2.1, there are disjoint paths $Q_{1}, Q_{2} \subseteq V\left(F^{*}\right)$, internally-disjoint from $S$, such that $Q_{1}$ is a $\left(p_{2}^{1}, p_{1}^{2}\right)$-path, and $Q_{2}$ is a $\left(p_{1}^{1}, p_{2}^{2}\right)$-path. As $\left|f^{*}\right| \leqslant 9,\left|Q_{1}\right|+\left|Q_{2}\right| \leqslant 9$. By $2.3(0),\left|Q_{1}\right| \geqslant 4$. Hence, $\left|Q_{2}\right| \leqslant 5$. But then we easily find an 8-cycle in $S \cup f^{*} \subseteq G$; a contradiction.

\subsection{Properties of a 5 -face not adjacent to a 3 -face}

Let $f \in F(G)$ be a 5 -face, which is not adjacent to a 3 -face. Let $x_{1}, x_{2}, x_{3}, x_{4}, x_{5}$ be the vertices of $f$ in a cyclic ordering. Let $y_{i}, 1 \leqslant i \leqslant 5$, be the neighbor of $x_{i}$ other than $x_{i-1}$ and $x_{i+1}$ (throughout $x_{0}:=x_{5}, x_{6}:=x_{1}, y_{0}:=y_{5}$, and $y_{6}:=y_{1}$ ).

2.12. For distinct $1 \leqslant i, j \leqslant 5, y_{i} \neq y_{j}$. 
Proof. For suppose not. Without loss of generality assume that $i<j$. If $j \neq i+1$, then $G$ contains a 4 -cycle. If $j=i+1$, then as $f$ is not adjacent to a 3 -face, it must be that $\operatorname{int} C=\left\{x_{i}, x_{i+1}, y_{i}\right\} \neq \emptyset$; but then $y_{i}$ is a cut-vertex. In both cases we obtain a contradiction to the definition of $G$.

Let $f_{i} \in F(G) \backslash\{f\}(i=1, \ldots, 5)$ such that $f_{i}$ is incident to $x_{i} x_{i+1}$. Suppose $f_{i}=$ $x_{i} y_{i} p_{1}^{i} p_{2}^{i} \ldots p_{\left|f_{i}\right|-4}^{i} y_{i+1} x_{i+1}$ and let $P^{i}=p_{1}^{i} p_{2}^{i} \ldots p_{\left|f_{i}\right|-4}^{i}$. By $2.3(0,2),\left|P^{i}\right| \geqslant 2(i=1, \ldots, 5)$.

2.13. Let $i$ and $j$ be distinct, $1 \leqslant i, j \leqslant 5$, and assume that $j \neq i+1$ and $j \neq i-1$. If $\left|f_{i}\right|=\left|f_{j}\right|=6$, then $f_{i}$ and $f_{j}$ are disjoint.

Proof. By symmetry assume that $i=1$ and $j=3$. Let $S=V(f) \cup\left\{y_{1}, \ldots, y_{5}\right\} \cup V\left(P^{1}\right)$. By $2.12,|S| \geqslant 10$. Next we show that $|S|=12$. As $f_{1}$ is adjacent to $f, f_{2}$ and $f_{5}$, then $V\left(P^{1}\right) \cap\left(V(f) \cup\left\{y_{3}, y_{5}\right\}\right)=\emptyset$. Now, if $|S|<12$, then $V\left(P^{1}\right) \cap\left\{y_{4}\right\} \neq \emptyset$ and $p_{1}^{1}=y_{4}$ or $p_{2}^{1}=y_{4}$, and by symmetry we may assume that $p_{2}^{1}=y_{4}$

Let $C:=x_{2} y_{2} y_{4} x_{4} x_{3}$. By symmetry we may assume that $y_{3} \in \operatorname{int}(C)$. If $\left(\Gamma\left(y_{2}\right) \cup\right.$ $\left.\Gamma\left(y_{4}\right)\right) \cap \operatorname{ext}(C)=\emptyset$, then $\left\{x_{1}, x_{5}\right\}$ is a 2-cut in $G$, separating $y_{3}$ and $y_{1}$. If $\left(\Gamma\left(y_{2}\right) \cup\right.$ $\left.\Gamma\left(y_{4}\right)\right) \cap \operatorname{int}(C)=\emptyset$, then $x_{3}$ is a cut-vertex in $G$, separating $y_{3}$ and $y_{1}$. Hence we have that $\Gamma\left(y_{2}\right) \cap \operatorname{int}(C)=\emptyset$ and $\Gamma\left(y_{4}\right) \cap \operatorname{int}(C) \neq \emptyset$ or $\Gamma\left(y_{4}\right) \cap \operatorname{int}(C)=\emptyset$ and $\Gamma\left(y_{2}\right) \cap \operatorname{int}(C) \neq \emptyset$. In the former case, $\left\{x_{3}, y_{4}\right\}$ is a 2 -cut in $G$ separating $y_{3}$ and $y_{1}$, and in the latter case, $\left\{x_{3}, y_{2}\right\}$ is a 2 -cut in $G$ separating $y_{3}$ and $y_{1}$. Hence $|S|=12$.

Now, let $v \in S \backslash\left\{y_{4}, x_{4}\right\}$. We see that $y_{4} v \notin E(G)$. For if $y_{4} v \in E(G)$, then by 2.12, $v \notin V(f) \backslash\left\{x_{4}\right\}$, and by 2.3(0), $v \cap\left\{y_{3}, y_{5}\right\}=\emptyset$. Hence, it must be that $v \in\left\{y_{1}, p_{1}^{1}, p_{2}^{1}, y_{2}\right\}$; but then it is easily seen that $G$ contains an 8-cycle.

Hence we have shown that $V\left(f_{1}\right) \cap\left\{p_{2}^{3}\right\}=\emptyset$, and together with the fact that $|S|=12$, we conclude that $V\left(f_{1}\right) \cap\left\{y_{4}, y_{3}, p_{2}^{3}\right\}=\emptyset$. Hence $\left|V\left(f_{1}\right) \cap V\left(f_{3}\right)\right| \leqslant 1$, and by 1.3 , $f_{1}$ and $f_{3}$ are disjoint.

2.14. Suppose that $\left|f_{i}\right| \geqslant 6$ (for some $1 \leqslant i \leqslant 5$ ). If $f$ is a $\left(6,6,6,6,\left|f_{i}\right|\right)$-face, then $\left|f_{i}\right|=6$ or $\left|f_{i}\right| \geqslant 10$.

Proof. By symmetry assume that $f_{i}=f_{1}$ (and then $\left|f_{j}\right|=6$, for $j=2, \ldots, 5$ ). Now, assume for a contradiction that $7 \leqslant\left|f_{i}\right| \leqslant 9$. Clearly, $\left|f_{i}\right| \neq 8$. Let $S=f \cup \bigcup_{i=2}^{5} f_{i}$. By 2.13, $|V(S)|=18$.

It must be that $f_{1}$ is adjacent both to $f_{3}$ and $f_{4}$, for otherwise one of $f_{1} \cup f \cup f_{3}$ or $f_{1} \cup f \cup f_{4}$ (if $\left|f_{1}\right|=9$ ) or one of $f \cup f_{1} \cup f_{2} \cup f_{4}$ or $f \cup f_{1} \cup f_{3} \cup f_{5}$ (if $|f|=7$ ) contains a 16-cycle. Hence, $f_{1}$ is semi-adjacent to $f$ via the edge $x_{4} y_{4}$. Let $Q_{1} \subseteq f_{1}$ (resp., $Q_{2} \subseteq f_{2}$ ) be the $p_{1}^{4} y_{1}$-path (resp., $p_{2}^{3} y_{2}$-path) on $f_{1}$. Since $\left|f_{1}\right| \leqslant 9$, there exist $1 \leqslant i \leqslant 2$ so that $\left|Q_{i}\right| \leqslant 4$. But then it is easily seen that $Q_{i} \cup S$ contains an 8-cycle; a contradiction.

To conclude this section, we need the following lemma, proof of which is similar to the proof of 2.14 .

2.15. Suppose $\left|f_{i}\right|=6$ (for $\left.i=1, \ldots, 5\right)$. Let $g_{i}$ be the face incident to $y_{i}$, other than $f_{i-1}$ and $f_{i+1}$ (that is $\left\{g_{1}, \ldots, g_{5}\right\}$ is the set of semi-adjacent faces of $f$ ). Then, $\left|g_{j}\right| \geqslant 10$, for $1 \leqslant j \leqslant 5$. 


\section{Chains and Clusters}

Let $f \in F(G)$ be a $k$-face. Let $x_{1}, \ldots, x_{k} \in V(f)$ be the vertices of $f$ in a cyclic clockwise ordering. Let $1 \leqslant \ell<k$, and let $\left\{f_{1}, \ldots, f_{\ell}\right\} \subseteq \Gamma_{G}(f)$. By 1.3, $\left|E(f) \cap E\left(f_{i}\right)\right|=1$, for $i=1, \ldots, \ell$. Let

$$
A=\left\{e \in E(f): \text { there exists } 1 \leqslant i \leqslant \ell \text { with } E(f) \cap E\left(f_{i}\right)=e\right\}
$$

We say that the faces $f_{1}, \ldots, f_{\ell}$ are consecutive on $f$, if $G[A]$ is a connected path (note that since $\ell<k, G[A]$ cannot be a cycle).

If $f_{1}, \ldots, f_{\ell}$ are consecutive on $f$, we write $f_{1} \prec_{f} f_{2} \prec_{f}, \ldots, \prec_{f} f_{\ell}$, if when traversing the edges of $f$ in a clockwise order starting from $E\left(f_{1}\right) \cap E(f), E\left(f_{i}\right) \cap E(f)$ is met before $E\left(f_{i+1}\right) \cap E(f)$, for $i=1, \ldots, \ell-1$.

Now, suppose $c:=\left\{f_{1}, \ldots, f_{\ell}\right\}$ is a set of consecutive faces on $f$. We say that $c$ is a chain of $f$, if for every $1 \leqslant i<j \leqslant \ell$ with $j \neq i+1$, we have that $f_{i}$ and $f_{j}$ are disjoint unless $j=i+2$ and $f_{i+1}$ is a 3 -face (and then $f_{i}$ and $f_{j}$ share an edge in common).

3.1. Let $c:=\left\{f_{1}, \ldots, f_{\ell}\right\}, 1 \leqslant \ell \leqslant k-2, f_{1} \prec_{f}, \ldots, \prec_{f} f_{\ell}$, be a chain of $f$. Let $g \in F(G) \backslash(\{f\} \cup c)$ be a 3-face, and suppose that $g$ and $f_{i}$ are consecutive on $f$, for some $i \in\{1, \ell\}$. Then, $c^{\prime}=f_{1} \cup \cdots \cup f_{\ell} \cup g$ is a chain of $f$.

Proof. By symmetry me may assume that $i=\ell$. Without loss of generality, we may assume that $E\left(f_{j}\right) \cap E(f)=\left\{x_{j} x_{j+1}\right\}, 1 \leqslant j \leqslant \ell$, and then $E(g) \cap E(f)=\left\{x_{\ell} x_{\ell+1}\right\}$. It suffices to show that for $j=1, \ldots, \ell-1, f_{j}$ and $g$ are disjoint. Indeed, if $V\left(f_{j}\right) \cap V(g) \neq \emptyset$ $(1 \leqslant j \leqslant \ell-1)$ then $f_{j}$ is adjacent to $g$, and hence, as $g$ is a 3 -face, also adjacent to $f$ on the edge $x_{\ell+1} x_{\ell+2}$. But then $\left\{x_{j} x_{j+1}, x_{\ell+1} x_{\ell+2}\right\} \subseteq E\left(f_{j}\right) \cap E(f)$, contradicting 1.3.

3.2. Let $c:=\left\{f_{1}, \ldots, f_{\ell}\right\} \subseteq \Gamma_{G}(f), 1 \leqslant \ell \leqslant k-1, f_{1} \prec_{f}, \ldots, \prec_{f} f_{\ell}$, be a set of consecutive faces on $f$. Then $c$ is a chain of $f$, if one of the following conditions holds:

1. $\ell \leqslant 2$.

2. $\ell=3$ and $\left(\left|f_{1}\right|,\left|f_{2}\right|,\left|f_{3}\right|\right) \stackrel{\leftrightarrow}{\in}\{(6,6,5),(6,5,6)\}$.

3. $\ell=4$ and $\left(\left|f_{1}\right|,\left|f_{2}\right|,\left|f_{3}\right|,\left|f_{4}\right|\right) \stackrel{\leftrightarrow}{\in}\{(6,3,6,5),(6,3,6,6)\}$.

4. $\ell=5$ and

$$
\left(\left|f_{1}\right|,\left|f_{2}\right|,\left|f_{3}\right|,\left|f_{4}\right|,\left|f_{5}\right|\right) \stackrel{\leftrightarrow}{\in}\{(5,6,3,6,5),(5,6,3,6,6),(6,3,6,5,6),(6,6,3,6,6)\}
$$

5. $\ell=6$ and $\left(\left|f_{1}\right|,\left|f_{2}\right|,\left|f_{3}\right|,\left|f_{4}\right|,\left|f_{5}\right|,\left|f_{6}\right|\right) \in\{(6,3,6,6,3,6)\}$.

(The notation $A \stackrel{\leftrightarrow}{\in} B$ means that either $A$ is in $B$, or the reversal of $A$ is in $B$, so that $(1,2) \stackrel{\leftrightarrow}{\in}\{(2,1)\}$.

Proof. We shall prove (1)-(2). Correctness of (3)-(5) follows by similar arguments. As in the proof of 3.1, we may assume, without loss of generality, that $E\left(f_{i}\right) \cap E(f)=\left\{x_{i} x_{i+1}\right\}$, for $1 \leqslant i \leqslant \ell$. Item $(1)$ follows immediately from 1.3 .

For the proof of $(2)$, assume that $\left(\left|f_{1}\right|,\left|f_{2}\right|,\left|f_{3}\right|\right)=(6,6,5)$ (if $\left(\left|f_{1}\right|,\left|f_{2}\right|,\left|f_{3}\right|\right)=(6,5,6)$ then the proof follows similar arguments). Let $x_{1}, u_{1}, u_{2}, u_{3}, u_{4}, x_{2} \in V\left(f_{1}\right)$ be the vertices of $f_{1}$ in a cyclic clockwise order. Similarly, let $x_{2}, u_{4}, u_{5}, u_{6}, u_{7}, x_{3}$ be the vertices of $f_{2}$ in a cyclic clockwise order. We need to show that $f_{1}$ and $f_{3}$ are disjoint. Suppose not. By 1.3, 
either $E\left(f_{1}\right) \cap E\left(f_{3}\right)=u_{1} u_{2}$ or $E\left(f_{1}\right) \cap E\left(f_{3}\right)=u_{2} u_{3}$. If $f_{1}$ and $f_{3}$ are both incident to $u_{2} u_{3}$, then $u_{2} x_{4} \in E(G)$; but then $x_{4} x_{3} u_{7} u_{6} u_{5} u_{4} u_{3} u_{2} x_{4}$ is an 8-cycle. If $f_{1}$ and $f_{3}$ are both incident to $u_{1} u_{2}$, then $u_{2} u_{7} \in E(G)$; but then $u_{7} u_{6} u_{5} u_{4} x_{2} x_{1} u_{1} u_{2} u_{7}$ is an 8-cycle. Both cases lead to a contradiction. Hence, $f_{1}$ and $f_{3}$ are disjoint as required.

3.2 and the assumption that $G$ contains no 16-cycles imply the following immediate corollary.

3.3. Let $f_{1}, \ldots, f_{\ell}, 1 \leqslant \ell \leqslant k-1, f_{1} \prec_{f}, \ldots, \prec_{f} f_{\ell}$, be a set of consecutive faces on $f$. Then, $\left(\left|f_{1}\right|, \ldots,\left|f_{\ell}\right|\right) \notin\{(5,6,3,6,6),(5,6,3,6,5),(6,3,6,5,6),(6,3,6,6,3,6)\}$.

\subsection{Partition into clusters}

We start by defining two sets, $\Im_{S}$ and $\Im_{C}$, of plane graphs which are depicted in Figures 2 and 3. (The specific embeddings as in the figures are important.) We identify the names of the graphs by the lengths of their (internal) faces. Set $\Im_{\text {all }}:=\Im_{S} \cup \Im_{C}$.

$$
\Im_{S}=\{(3),(3,5),(5),(3,5,3),(6,3,6),(6,3,6,5),(3,6,6,3),
$$

$(3,5,6,3),(6,3,6,6,3),(6,3,6,5,3),(3,6),(3,6,5),(9,3,5,3),(3,9,3,5,3)$,

$$
(3,9,3,5),(5,3,9,3,5),(3,6,6,3,6,6,3),(3,6,5,6,3),(9,3,6),(3,9,3,6)\}
$$

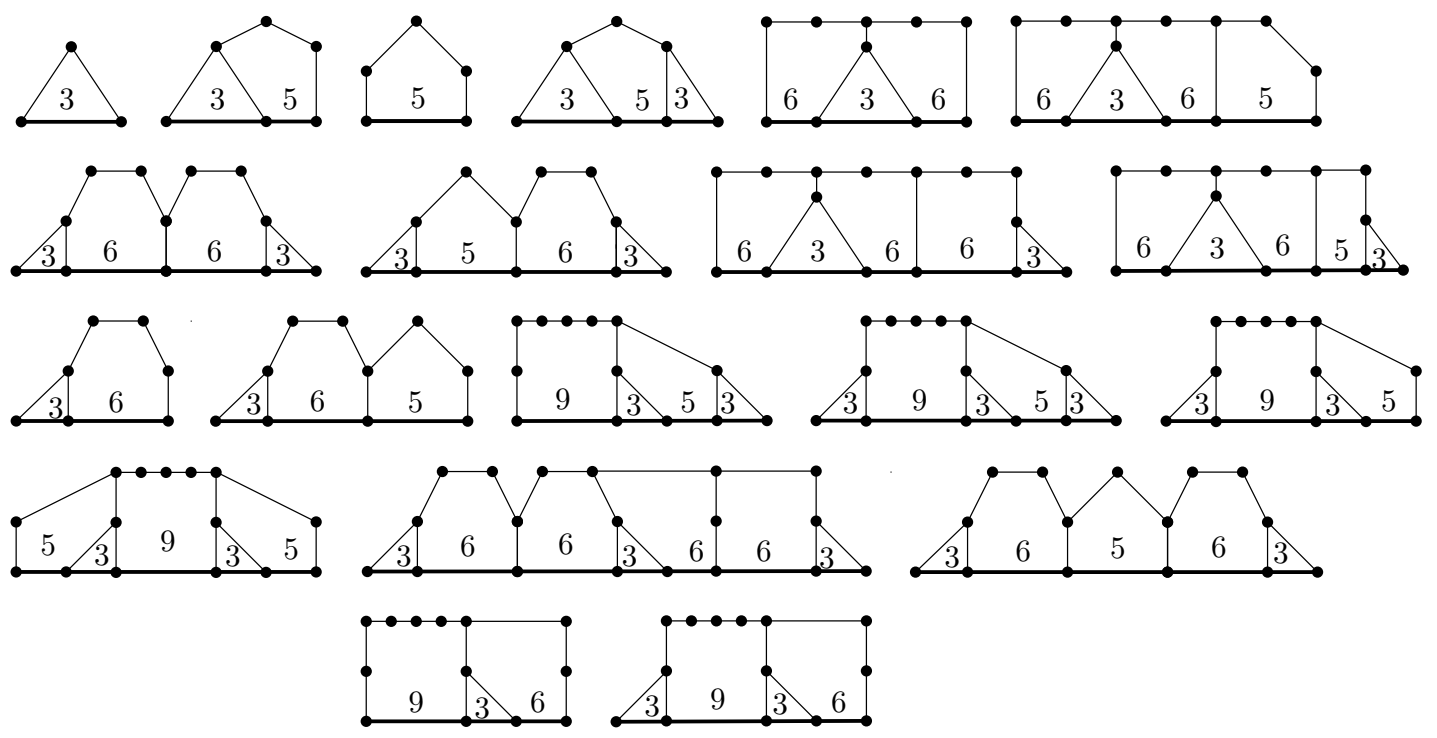

Figure 2: The set $\Im_{S}$.

$$
\begin{gathered}
\Im_{C}=\left\{\left(3,5^{3}\right),\left(6^{3} 6\right),\left(6^{3} 6,5\right),\left(6^{3} 6,5,3\right),\left(6^{656} 6\right),\left(6^{6} 5^{6} 6\right),\left(6^{3} 6,6,3\right),\right. \\
\left(9,3,5^{3}\right),\left(3,9,3,5^{3}\right),\left(5,9,3,5^{3}\right),\left(5,3,9,3,5^{3}\right),\left(5^{3}, 3,9,3,5^{3}\right),\left(9,5^{3}, 3\right), \\
\left.\left(3,9,5^{3}, 3\right),\left(5^{3}, 3,9,5,9,3,5^{3}\right),\left(6^{3} 6\right)\right\}
\end{gathered}
$$

It will be convenient to partition $\Im_{C}$ into the following sets (see Figure 3 ).

$$
\Im_{C}^{3}=\left\{\left(3,5^{3}\right)\right\}
$$



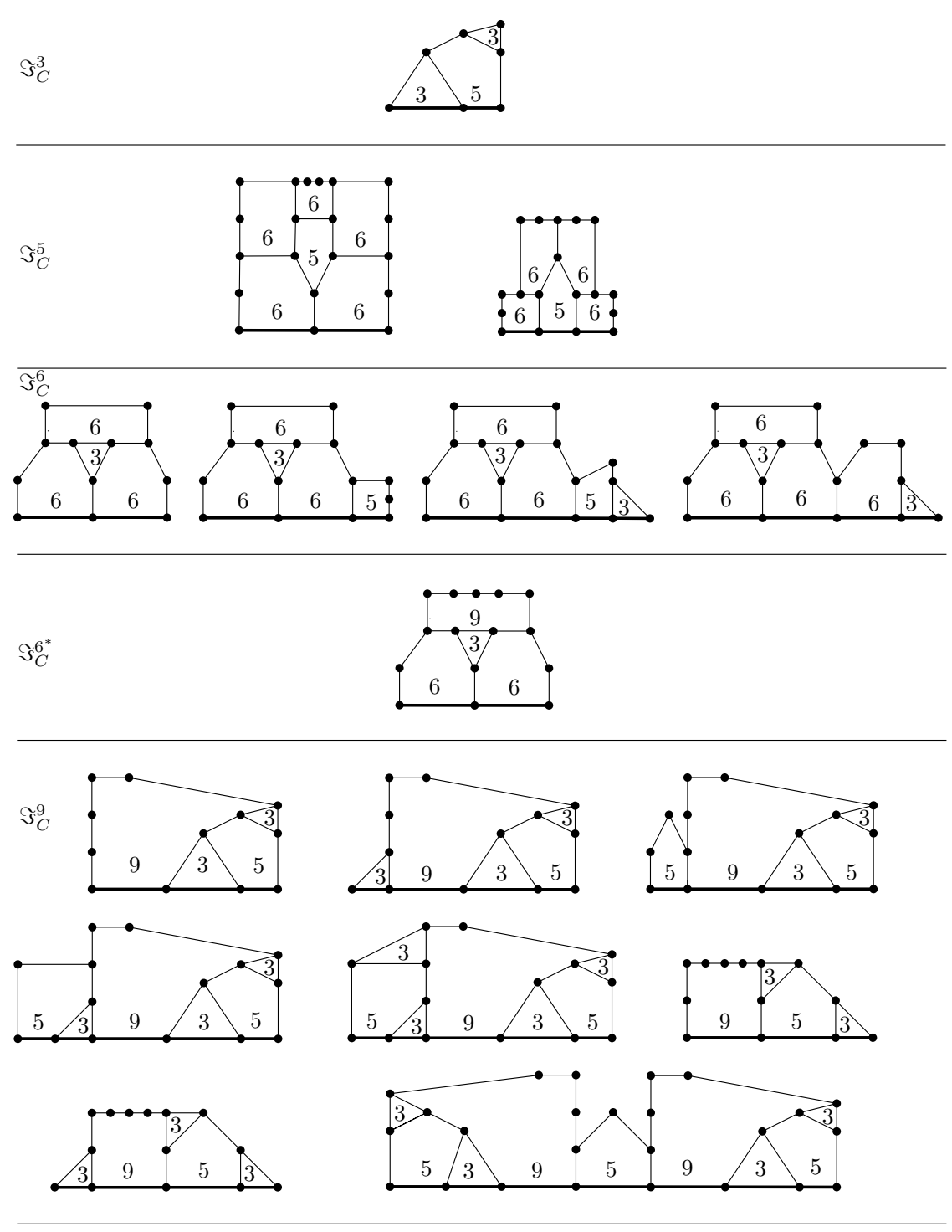

Figure 3: The set $\Im_{C}$.

$$
\begin{gathered}
\Im_{C}^{5}=\left\{\left(6^{656} 6\right),\left(6^{6} 5^{6} 6\right)\right\} \\
\Im_{C}^{6}=\left\{\left(6^{3} 6\right),\left(6^{6} 6,5\right),\left(6^{3} 6,5,3\right),\left(6^{3} 6,6,3\right)\right\} \\
\Im_{C}^{6^{*}}=\left\{\left(6^{3} 6\right)\right\} \\
\Im_{C}^{9}=\left\{\left(9,3,5^{3}\right),\left(3,9,3,5^{3}\right),\left(5,9,3,5^{3}\right),\left(5,3,9,3,5^{3}\right),\left(5^{3}, 3,9,3,5^{3}\right),\right. \\
\left.\left(9,5^{3}, 3\right),\left(3,9,5^{3}, 3\right),\left(5^{3}, 3,9,5,9,3,5^{3}\right)\right\}
\end{gathered}
$$

Next three additional subsets of $\Im_{\text {all }}$ are defined.

$$
\begin{gathered}
\Im^{9}=\Im_{C}^{9} \cup\{(9,3,5,3),(3,9,3,5,3),(3,9,3,5),(5,3,9,3,5),(9,3,6),(3,9,3,6)\} \\
\Im_{F}=\Im_{\text {all }} \backslash\left\{(5),\left(6^{3} 6\right),\left(6^{3} 6,5\right),\left(6^{3} 6\right),\left(6^{656} 6\right),\left(6^{6} 5^{6} 6\right)\right\}
\end{gathered}
$$




$$
\Im_{P}=\Im_{\text {all }} \backslash\left\{(5),\left(6^{3} 6\right),\left(6^{3} 6,5\right),\left(6^{3} 6\right),\left(6^{656} 6\right),\left(6^{6} 5^{6} 6\right),(3,6),(3,6,5)\right\}
$$

Note that

$$
\Im_{P} \subseteq \Im_{F}
$$

and

$$
\Im_{F} \backslash \Im_{P}=\{(3,6),(3,6,5)\}
$$

For each $X \in \Im_{\text {all }}$ we define a unique path $P(X) \subseteq X$. The paths are depicted in bold in Figures 2 and 3.

Let $H \subseteq G$ (together with its induced embedding in $G$ ) and let $f \in F(G)$. We say that $H$ is a cluster of $f$ of type $X$, if the following holds:

1. $H \cong X$, for some $X \in \Im_{\text {all }}$.

2. $H$ is a union of faces of $G$, each distinct from $f$.

3. $P(H) \subseteq f$.

Let $H \subseteq G, f \in F(G)$, and suppose that $H$ satisfies (1)-(3) with respect to $f$. The type of $H$ is denoted by $t(H)$ (then $t(H) \in \Im_{\text {all }}$ ). We denote by $F(H)$ the set of all faces of $H$ excluding the unique face of $H$ which contains $P(H)$ entirely (note that this face is not a face of $G$ as it has vertices of degree two on its boundary). We denote by Chain $(H)$, the set of faces of $H$ that have at least one edge in common with $P(H)$, excluding the unique face of $H$ which contains $P(H)$. The reader may verify by inspection of $\Im_{\text {all }}$ that Chain $(H)$ is a chain of $f$.

For $f \in F(G)$, define $\Upsilon_{f}$ to be the set of all clusters of $f$. Note that distinct clusters in $\Upsilon_{f}$ may not be disjoint.

Next we eliminate redundancies in $\Upsilon_{f}$. This is done by constructing a set $\Pi_{f} \subseteq \Upsilon_{f}$ that captures the structure of $\Upsilon_{f}$, but in which the clusters are pairwise disjoint as much as possible. Let $Z \subseteq \Gamma_{G}(f)$ be such that $g \in Z$ if and only if there exist $c \in \Upsilon_{f}$ with $g \in F(c)$. Define $\Pi_{f} \subseteq \Upsilon_{f}$ so that the following conditions holds:

- For every $g \in Z$, there exists $c \in \Pi_{f}$ with $g \in F(c)$.

- If $c \in \Pi_{f}$, then $F(c) \nsubseteq F\left(c^{\prime}\right)$ for every $c^{\prime} \in \Upsilon_{f}$ distinct from $c$.

Clearly, $\Pi_{f}$ is well-defined.

To avoid repetition, let us extract the following short hypothesis, common to many statements that follow.

Hypothesis A. Let $f$ be a $k$-face, $k \geqslant 9$. Let $x_{1}, \ldots, x_{k} \in V(f)$ be the vertices of $f$ in a cyclic order. Let $\Upsilon_{f}$ and $\Pi_{f}$ be as defined above.

The following is the main lemma of this section. It asserts that under certain conditions the clusters in $\Pi_{f}$ are "almost" pairwise disjoint.

3.4. Under Hypothesis $A$, let $c_{1}, c_{2} \in \Pi_{f}$ be distinct. Then,

1. $P\left(c_{1}\right)$ and $P\left(c_{2}\right)$ are disjoint. 
2. If $t\left(c_{1}\right), t\left(c_{2}\right) \in \Im_{F}$ and $k \in\{9,10,17,18\}$, then $c_{1}$ and $c_{2}$ are disjoint unless $t\left(c_{i}\right)=$ $\left(5,9,3,5^{3}\right)$, for some $1 \leqslant i \leqslant 2$, and then $c_{3-i}$ is disjoint from $c_{i}^{\prime}$, where $c_{i}^{\prime} \subseteq c_{i}$ is a sub-cluster of $c_{i}$ of type $\left(9,3,5^{3}\right)$.

3. If $t\left(c_{1}\right), t\left(c_{2}\right) \in \Im_{P}$, then $c_{1}$ and $c_{2}$ are disjoint, unless $t\left(c_{1}\right), t\left(c_{2}\right)=(6,3,6)$ or $t\left(c_{i}\right)=\left(5,9,3,5^{3}\right)$, for some $1 \leqslant i \leqslant 2$. In the latter cases $c_{3-i}$ is disjoint from $c_{i}^{\prime}$, where $c_{i}^{\prime} \subseteq c_{i}$ is the sub-cluster of $c_{i}$ of type $\left(9,3,5^{3}\right)$.

4. If $t\left(c_{1}\right) \in\left\{\left(6^{3} 6\right),\left(6^{3} 6,5\right),\left(6^{3} 6\right)\right\}$, then Chain $\left(c_{1}\right)$ and $c_{2}$ are disjoint.

5. Suppose $c_{1}, c_{2}, \ldots, c_{m} \in \Pi_{f}$ and $t\left(c_{i}\right)=(6,3,6)(i=1, \ldots, m)$. Then each $c_{i}(i=$ $1, \ldots, m)$ contains a sub-cluster $\hat{c}_{i}$ of type $(3,6)$ such that for distinct $1 \leqslant j, r \leqslant m$, $\hat{c}_{j}$ and $\hat{c}_{r}$ are disjoint.

3.4 is proved via a series of claims. We start with the following claim which greatly facilitates the proof of 3.4 .

3.5. Under Hypothesis $A$, let $c_{1}, c_{2} \in \Pi_{f}$ be distinct and suppose $P\left(c_{1}\right)$ and $P\left(c_{2}\right)$ are disjoint. Then, $c_{1}$ and $c_{2}$ are disjoint provided that one of the following holds:

1. $t\left(c_{1}\right)=(3,6)$ and $t\left(c_{2}\right)=(3,5)$, or $k \in\{9,10,17,18\}$ and $t\left(c_{1}\right), t\left(c_{2}\right)=(3,6)$

2. $k \in\{9,10,17,18\}, t\left(c_{1}\right)=(3,6)$, and $t\left(c_{2}\right)=(3,6,5)$.

Proof. Let $F\left(c_{1}\right)=\left\{g_{1}, g_{2}\right\}$ such that $\left|g_{1}\right|=3$ and $\left|g_{2}\right|=6$. Assume, without loss of generality, that $V\left(g_{1}\right) \cap V(f)=\left\{x_{1}, x_{2}\right\}$ and $V\left(g_{2}\right) \cap V(f)=\left\{x_{2}, x_{3}\right\}$. Let $\left\{u_{1}, \ldots, u_{4}\right\} \subseteq$ $V\left(g_{2}\right)$ such that $u_{1} x_{2}, u_{4} x_{3} \in E\left(g_{2}\right)$ and $u_{i} u_{i+1} \in E\left(g_{2}\right)$, for $1 \leqslant i \leqslant 3$. Note that $V\left(g_{1}\right)=\left\{x_{1}, u_{1}, x_{2}\right\}$.

(1). Assume for a contradiction that the claim is false. Let $F\left(c_{2}\right)=\left\{f_{1}, f_{2}\right\}$ such that $\left|f_{1}\right|=3$ and $\left|f_{2}\right| \in\{5,6\}$. Since $P\left(c_{1}\right)$ and $P\left(c_{2}\right)$ are disjoint, $E(f) \cap\left(E\left(f_{1}\right) \cup E\left(f_{2}\right)\right)=$ $\left\{x_{j} x_{j+1}, x_{j+1} x_{j+2}\right\}$, for some $4 \leqslant j \leqslant k-1$.

Two cases are possible. Either $E(f) \cap E\left(f_{1}\right)=\left\{x_{j} x_{j+1}\right\}$ or $E(f) \cap E\left(f_{1}\right)=\left\{x_{j+1} x_{j+2}\right\}$. We prove the former case. The latter case is resolved using the exact same argument.

Let $v \in V\left(f_{1}\right)$, other than $x_{j}$ and $x_{j+1}$. Since $P\left(c_{1}\right)$ and $P\left(c_{2}\right)$ are disjoint, $f_{1} \cap$ $\left(g_{1} \cup g_{2}\right)=\emptyset$. Hence $V\left(c_{1}\right) \cap V\left(c_{2}\right) \subseteq V\left(f_{2}\right)$, and together with 1.3, it follows that $\left|E\left(c_{1}\right) \cap E\left(c_{2}\right)\right|=1$. Since $P\left(c_{1}\right)$ and $P\left(c_{2}\right)$ are disjoint and $g_{1}$ is a 3 -face, $f_{2}$ is adjacent to $g_{2}$ on the edge $u_{2} u_{3}$ or $u_{3} u_{4}$.

We show that $\left|f_{2}\right| \neq 5$. For suppose $\left|f_{2}\right|=5$. If $f_{2}$ and $g_{2}$ are adjacent on $u_{2} u_{3}$, then by $2.1, v u_{3}, x_{j+2} u_{2} \in E(G)$. Let $C:=x_{j+2} x_{j+3} \ldots x_{k} x_{1} u_{1} u_{2} x_{j+2}$. If $x_{j+2}=x_{k}$, then $C$ is a 4-cycle, otherwise $\left\{x_{j+2}, x_{1}\right\}$ is a 2-cut; a contradiction. If $f_{2}$ and $g_{2}$ are adjacent on $u_{3} u_{4}$, then by $2.1, v u_{4}, x_{j+2} u_{4} \in E(G)$. Let $C:=u_{4} x_{3} x_{4} \ldots x_{j} v u_{4}$. If $j=4$, then $C$ is a 4-cycle, otherwise, $\left\{x_{3}, x_{j}\right\}$ is a 2-cut; all cases lead to a contradiction, and hence $\left|f_{2}\right| \neq 5$.

Suppose then that $\left|f_{2}\right|=6$ and $k \in\{9,10,17,18\}$. Let $S=f \cup g_{1} \cup g_{2} \cup f_{1}$. Let $Q_{1}$ and $Q_{2}$ be two paths such that $V\left(Q_{1}\right), V\left(Q_{2}\right) \subseteq V(f), Q_{1}=x_{j+3} \ldots x_{k}$, and $Q_{2}=x_{4} \ldots x_{j-1}$ (possibly $Q_{1}$ or $Q_{2}$ are empty).

Case 1. Suppose that $E\left(f_{2}\right) \cap E\left(g_{2}\right)=\left\{u_{2} u_{3}\right\}$. First we see that $x_{j+2} u_{2} \notin E(G)$. Indeed, if $x_{j+2} u_{2} \in E(G)$, let $C:=x_{j+2} x_{j+3} \ldots x_{k} x_{1} u_{1} u_{2} x_{j+2}$. If $Q_{1}$ is empty, then $x_{j+2}=$ $x_{k}$, and $C$ is a 4 -cycle. If $\left|Q_{1}\right| \geqslant 1$, then $\left\{x_{j+2} x_{1}\right\}$ is a 2 -cut. Hence, $x_{j+2} u_{2} \notin E(G)$. By 1.3 and as $\left|f_{2}\right|=6$, it follows that $u_{3} v \in E(G)$ and there is $z \in V\left(f_{2}\right)$, such that $z \notin V(S)$ 
and $z u_{2}, z x_{j+2} \in E(G)$. As $k \in\{9,10,17,18\}$, then $\left|Q_{1}\right|+\left|Q_{2}\right| \in\{3,4,11,12\}$. By 2.3(0), it is easily seen that that $\left|Q_{1}\right| \in\{1,4,5,12\}$. Hence, as $\left|Q_{1}\right|+\left|Q_{2}\right| \in\{3,4,11,12\}$, $\left|Q_{2}\right| \in\{0,1,2,3,6,7,8,10,11\}$. But then it can be easily verified that for each possible value of $V\left(Q_{2}\right), G$ contains an 8- or 16-cycle; a contradiction.

Case 2. Assume that $E\left(f_{2}\right) \cap E\left(g_{2}\right)=\left\{u_{3} u_{4}\right\}$. As in Case (1), we first show that $v u_{4} \notin E(G)$. Indeed, if $v u_{4} \in E(G)$, then let $C=u_{4} x_{3} x_{4} \ldots x_{j} v$. If $Q_{1}$ is empty, then $x_{j}=x_{4}$ and $C$ is a 4-cycle. If $\left|Q_{1}\right| \geqslant 1$ then $\left\{x_{3} x_{j+1}\right\}$ is a 2-cut. Hence, $v u_{4} \notin E(G)$. By 1.3 and as $\left|f_{2}\right|=6$, it follows that $u_{3} x_{j+2} \in E(G)$, and there is $z \in V\left(f_{2}\right)$ such that $z \notin$ $V(S)$, and $z u_{4}, z v \in E(G)$. As $k \in\{9,10,17,18\}\left|Q_{1}\right|+\left|Q_{2}\right| \in\{3,4,11,12\}$. By 2.3(0), it is easily seen that that $\left|Q_{1}\right| \in\{4,12\}$. Hence, as $\left|Q_{1}\right| \in\{4,12\},\left|Q_{2}\right| \in\{0,7,8\}$, and again it is easy to find an 8- or a 16-cycle in $G$; a contradiction.

(2) This part follows similar arguments as the proof of (1).

3.6. Under Hypothesis $A$, let $c_{1}, c_{2} \in \Pi_{f}$ be distinct, and suppose that $P\left(c_{1}\right)$ and $P\left(c_{2}\right)$ are disjoint. Then $c_{1}$ and $c_{2}$ are disjoint provided that one of the following conditions holds:

1. $t\left(c_{1}\right)=(6,3,6,5)$ and $t\left(c_{2}\right) \in\{(3,6),(3,6,5)\}$.

2. $t\left(c_{1}\right)=(3,6,6,3)$ and $t\left(c_{2}\right) \in\{(6,3,6),(3,6,6,3),(3,6,6,3,6,6,3)\}$.

3. $t\left(c_{1}\right) \in\{(3,6,5,3),(3,6,5,6,3)\}$ and $t\left(c_{2}\right) \in\{(3,5),(3,6)\}$.

4. $t\left(c_{1}\right)=(6,3,6,6,3)$ and $t\left(c_{2}\right) \in\{(6,3,6),(3,6,6,3)\}$.

Proof. Let $F\left(c_{1}\right)=\left\{f_{1}, \ldots, f_{\ell}\right\}$ such that $\ell=\left|F\left(c_{1}\right)\right|$. Assume, without loss of generality, that $E(f) \cap E\left(f_{i}\right)=\left\{x_{i} x_{i+1}\right\}$, for $1 \leqslant i \leqslant \ell$. By symmetry, we may assume that if $c_{1}$ is of type $\left(a_{1}, \ldots, a_{\ell}\right)$, then $\left|f_{i}\right|=a_{i}$, for $1 \leqslant i \leqslant \ell$. We prove Item (1). Items (2)-(4) are proved in a similar way.

Assume for a contradiction that (1) holds, but $V\left(c_{1}\right) \cap V\left(c_{2}\right) \neq \emptyset$. Let $g \in F\left(c_{2}\right)$ such that $V(g) \cap V\left(c_{1}\right) \neq \emptyset$. Clearly, disjointness of $P\left(c_{1}\right)$ and $P\left(c_{2}\right)$ imply that $F\left(c_{1}\right) \cap F\left(c_{2}\right)=$ $\emptyset$. We may also assume that $|g| \neq 3$. For if $|g|=3$, then it must be that $V(g) \cap V\left(f_{4}\right) \neq \emptyset$. But then we get a contradiction to 1.3, unless $f_{4}$ and $g$ are consecutive of $f$. But this is impossible as $P\left(c_{1}\right)$ and $P\left(c_{2}\right)$ are disjoint.

First we describe the settings. Let $u_{1}, \ldots, u_{4} \subseteq V\left(f_{1}\right)$ such that $u_{1} x_{1}, u_{4} x_{2} \in E(f)$ and $u_{i} u_{i+1} \in E\left(f_{1}\right), 1 \leqslant i \leqslant 3$. Let $u_{4}, u_{3}, u_{5}, u_{6} \subseteq V\left(f_{3}\right)$ such that $u_{6} x_{4}, u_{3} u_{5}, u_{5} u_{6} \in E\left(f_{3}\right)$. Let $u_{7}, u_{8} \subseteq V\left(f_{4}\right)$ such that $u_{6} u_{7}, u_{7} u_{8}, u_{8} x_{5} \in E\left(f_{4}\right)$. Let $g_{1}, g_{2} \in F(G)$ and if $t\left(c_{2}\right)=$ $(3,6,5)$ let also $g_{3} \in F(G)$ so that: $g_{1}, g_{2}$ or $g_{1}, g_{2}, g_{3}$ (if $g_{3}$ is defined) are consecutive on $f, F\left(c_{2}\right) \subseteq\left\{g_{1}, g_{2}, g_{3}\right\},\left|g_{1}\right|=3,\left|g_{2}\right|=6$, and if $t\left(c_{2}\right)=(3,6,5)$, then $\left|g_{3}\right|=5$.

We may assume that $\left|V(g) \cap V\left(c_{1}\right)\right| \neq 2$. For if $\left|V(g) \cap V\left(c_{1}\right)\right|=2$, then $E(g) \cap E\left(c_{1}\right) \in$ $\left\{u_{1} u_{2}, u_{7} u_{8}\right\}$, and $|g| \in\{5,6\}, c_{1} \cup g \subseteq G$ contains a 16-cycle. Hence, $\left|V(g) \cap V\left(c_{1}\right)\right| \geqslant 3$. Since $g \neq g_{1}$ and $P\left(c_{1}\right)$ and $P\left(c_{2}\right)$ are disjoint, we see that $\left|V(g) \cap V\left(c_{1}\right)\right|=3$. By 1.3 and as $u_{3}$ and $u_{6}$ are 3 -vertices in $G\left[E\left(c_{1}\right)\right]$, then $V(g) \cap V(c)=\left\{u_{2}, u_{3}, u_{5}\right\}$ or $V(g) \cap V(c)=$ $\left\{u_{5}, u_{6}, u_{7}\right\}$. We see that $g_{2}$ and $c_{1}$ are disjoint (for otherwise $c_{1} \cup g_{1} \cup g_{2}$ contains a 16-cycle). Hence, if $t\left(c_{2}\right)=(3,6)$ the claim follows. If $t\left(c_{2}\right)=(3,6,5)$ then by $2.3(2)$, $V\left(g_{3}\right) \cap V\left(c_{1}\right)=\left\{u_{2}, u_{3}, u_{4}\right\}$. But then, by 2.2(1), $g_{3} \cup g_{2} \cup f_{3} \cup f_{4}$ contains a 16-cycle; a contradiction. This contradiction concludes the proof.

By the definition of $\Im_{\text {all }}$ and $\Pi_{f}$ we have the following:

3.7. Let $c_{1}, c_{2} \in \Pi_{f}$ be distinct and suppose $V\left(c_{1}\right) \cap V\left(c_{2}\right) \neq \emptyset$. Then, $F\left(c_{2}\right) \nsubseteq F\left(c_{1}\right)$ and there exists $g \in F\left(c_{2}\right) \backslash F\left(c_{1}\right),|g| \in\{3,5,6,9\}$, so that $V\left(c_{1}\right) \cap V(g) \neq \emptyset$. 
The rest of this section is devoted to studying intersections of clusters in $\Pi_{f}$.

3.8. Under Hypothesis $A$, let $c_{1}, c_{2} \in \Pi_{f}$ be distinct, and suppose $t\left(c_{1}\right) \in \Im_{C}^{5}$. Then $c_{1}$ and $c_{2}$ are disjoint.

Proof. We shall assume that $t\left(c_{1}\right)=\left(6^{6} 5^{6} 6\right)$, as the proof when $t\left(c_{1}\right)=\left(6^{656} 6\right)$ is resolved using similar arguments.

Assume for a contradiction that $t\left(c_{1}\right)=\left(6^{6} 5^{6} 6\right)$ but $V\left(c_{1}\right) \cap V\left(c_{2}\right) \neq \emptyset$. Let $F\left(c_{1}\right)=$ $\left\{f_{1}, \ldots, f_{5}\right\}$ such that Chain $\left(c_{1}\right)=\left\{f_{1}, f_{2}, f_{3}\right\},\left|f_{1}\right|=\left|f_{3}\right|=\left|f_{4}\right|=\left|f_{5}\right|=6$ and $\left|f_{2}\right|=5$. Let $g \in F\left(c_{2}\right)$ as exists by 3.7. We may assume that $|g| \in\{5,6\}$, for by 1.3 and 2.2(1), $|g| \neq 9$ and $|g| \neq 3$. Let $f^{\prime} \in\left\{f_{1}, f_{3}, f_{4}, f_{5}\right\}$ so that $g$ and $f^{\prime}$ are adjacent.

Suppose $|g|=5$. Observe that $g$ must be disjoint from at least one 6 -face $f^{\prime \prime} \in$ $\left\{f_{1}, f_{3}, f_{4}, f_{5}\right\} \backslash f^{\prime}$. But then $g \cup f^{\prime} \cup f_{2} \cup f^{\prime \prime}$ contains a 16-cycle; a contradiction.

Suppose $|g|=6$. Since $t\left(c_{2}\right) \in \Im_{\text {all }}$, by inspection of $\Im_{\text {all }}$, there exists $g_{1} \in F\left(c_{2}\right)$, so that $\left|g_{1}\right| \in\{3,5\}$, and $g$ and $g_{1}$ are adjacent. Clearly $g_{1} \neq f_{2}$, and we may further assume that $g_{1}$ is disjoint from $c_{2}$, for otherwise, a contradiction is obtained as in the previous case above with $g_{1}$ playing the rule of $g$. If $\left|g_{1}\right|=5$ then $g \cup g_{1} \cup f^{\prime} \cup f_{2}$ contains a 16-cycle. If $\left|g_{1}\right|=3$, then it is straightforward to verify that $c_{2} \cup g \cup g_{1}$ contains a 16-cycle. Hence, both cases lead to a contradiction.

3.9. Under Hypothesis $A$, let $c_{1}, c_{2} \in \Pi_{f}$ be distinct and suppose $t\left(c_{1}\right)=\left(6^{3} 6\right)$. Then $c_{1}$ and $c_{2}$ are disjoint, unless $t\left(c_{2}\right)=(3)$ and then $P\left(c_{1}\right)$ and $P\left(c_{2}\right)$ are disjoint.

Proof. Let $F\left(c_{2}\right)=\left\{f_{1}, \ldots, f_{4}\right\}$ such that $\left|f_{1}\right|=\left|f_{2}\right|=6,\left|f_{3}\right|=3$ and $\left|f_{4}\right|=9$. If $t\left(c_{2}\right)=(3)$, then the claim follows by 2.3(3), and the fact that by the definition of $\Pi_{f}$, $F\left(c_{2}\right) \nsubseteq F\left(c_{1}\right)$.

Hence, we may assume that $t\left(c_{2}\right) \neq(3)$. Now assume for a contradiction $V\left(c_{1}\right) \cap$ $V\left(c_{2}\right) \neq \emptyset$. Let $g \in F\left(c_{2}\right)$ as exists by 3.7. By 2.11, $|g|=3$. By $2.3(3), V(g) \cap V\left(c_{1}\right)=$ $V(g) \cap V\left(f_{4}\right)$. As $t\left(c_{2}\right) \neq(3)$, there exists $g_{1} \in F\left(c_{2}\right),\left|g_{1}\right| \in\{5,6\}$, such that $g_{1}$ and $g$ are adjacent. As $g \notin F\left(c_{1}\right)$, then by $2.3(3), g_{1} \notin F\left(c_{1}\right)$. As $g$ is a 3 -face, then $g_{1}$ and $f_{4}$ are adjacent. But then $V\left(c_{1}\right) \cap V\left(g_{1}\right) \neq \emptyset$; contradicting 2.11.

3.10. Under Hypothesis $A$, let $c_{1}, c_{2} \in \Pi_{f}$ be distinct, and suppose that $t\left(c_{1}\right)=\left(3,5^{3}\right)$. Then, $c_{1}$ and $c_{2}$ are disjoint.

Proof. Suppose not. Let $g \in F\left(c_{2}\right)$ as exists by 3.7. By 2.3(1,5,6), $|g|=9$. By 1.3, there is a cluster of $f, c^{\prime}:=g \cup c_{1} \in\left\{\left(9,3,5^{3}\right),\left(3,5^{3}, 9\right)\right\}$, so that $t\left(c_{1}\right) \in \Im_{\text {all }}$ and $F\left(c_{1}\right) \subseteq F\left(c^{\prime}\right)$; contradicting the definition of $\Pi_{f}$.

3.11. Under Hypothesis $A$, let $c_{1}, c_{2} \in \Pi_{f}$ be distinct and suppose $t\left(c_{1}\right) \in \Im^{9}$. Then $c_{1}$ and $c_{2}$ are disjoint, unless one of the following holds:

- $t\left(c_{1}\right)=\left(5,9,3,5^{3}\right)$; and then $P\left(c_{1}\right)$ and $P\left(c_{2}\right)$ are disjoint, and if $c^{\prime}$ is the sub-cluster of $c$ of type $\left(9,3,5^{3}\right)$, then $c_{1}$ and $c_{2}$ are disjoint.

- $t\left(c_{2}\right)=(5)$; and then $P\left(c_{1}\right)$ and $P\left(c_{2}\right)$ are disjoint.

Proof. Assume for a contradiction $V\left(c_{1}\right) \cap V\left(c_{2}\right) \neq \emptyset$. By 3.8, 3.9, and 3.10, $t\left(c_{2}\right) \notin$ $\Im_{C}^{3} \cup \Im_{C}^{5} \cup \Im_{C}^{6^{*}}$.

Case 1. Suppose

$$
t\left(c_{1}\right) \in T_{1}:=\left\{(9,3,5,3),(3,9,3,5,3),\left(3,9,5^{3}, 3\right),\left(9,3,5^{3}\right),\left(3,9,3,5^{3}\right),\right.
$$




$$
\left.\left(5^{3}, 3,9,3,5^{3}\right),\left(5^{3}, 3,9,5,9,3,5^{3}\right)\right\}
$$

By 2.2(1), 2.3 and the definition of $\Pi_{f}$, it can be easily verified that

(1.i) $c_{1}$ is disjoint from $f^{\prime}$, for any $f^{\prime} \in F(G) \backslash F\left(c_{1}\right)$, with $\left|f^{\prime}\right| \in\{5,6,9\}$.

By $(1 . \mathrm{i}), t\left(c_{2}\right) \neq(5)$. Let $g \in F\left(c_{2}\right)$ as exists by 3.7. By (1.i), $|g|=3$. If $t\left(c_{2}\right)=(3)$, then $F\left(c_{2}\right)=\{g\}$ and $g$ is adjacent to $f$. Hence, by $1.3, g$ and $F(c)$ are consecutive on $f$. By 2.3(1), we deduce that $t\left(c_{1}\right) \in\left\{(9,3,5,3),\left(9,3,5^{3}\right)\right\}$. But then by 3.1 and 2.3(1), $c^{\prime}:=g \cup c_{1}$ is a cluster of $f, F\left(c_{1}\right) \subseteq F\left(c^{\prime}\right)$, and $t\left(c^{\prime}\right) \in\left\{(3,9,3,5,3),\left(3,9,3,5^{3}\right)\right\} \subseteq \Im_{\text {all }}$; contradicting the definition of $\Pi_{f}$. If $t\left(c_{2}\right) \neq(3)$, then $\left|F\left(c_{2}\right)\right| \geqslant 2$. By inspection of $\Im_{\text {all }}$, there is $g_{1} \in F\left(c_{2}\right)$ such that $g_{1} \in\{5,6\}, g$ and $g_{1}$ are adjacent and $g_{1}$ and $f$ are adjacent. We see that $g_{1} \notin F\left(c_{1}\right)$; for otherwise $\left|g_{1}\right|=5$ and by 2.3(1), it must be that $g \in F\left(c_{1}\right)$, contradicting the definition of $g$. Hence, $g_{1} \notin F\left(c_{1}\right)$. But $g$ being a 3 -face intersecting both $c_{1}$ and $g_{1}$ implies that $V\left(g_{1}\right) \cap V\left(c_{1}\right) \neq \emptyset$; contradicting (1.i).

Case 2. Suppose that: $t\left(c_{1}\right) \in T_{2}:=\left\{(5,3,9,3,5),\left(5,3,9,3,5^{3}\right)\right\}$.

As in Case (1), we first see that

(2.i) $c_{1}$ is disjoint from $f^{\prime}$, for any $f^{\prime} \in F(G) \backslash F\left(c_{1}\right)$, with $\left|f^{\prime}\right| \in\{5,6\}$.

By $(2 . \mathrm{i}), t\left(c_{2}\right) \neq(5)$. We may assume that $t\left(c_{2}\right) \notin T_{1}$ (for otherwise the proof proceeds as in Case (1), with $c_{2}$ playing the rule of $c_{1}$ ).

Let $g \in F\left(c_{2}\right)$ as exists by 3.7. By (2.i), $|g|=3$ or $|g|=9$. If $|g|=3$, the proof follows by the same arguments as in Case (1). Hence, assume that $|g|=9$. By 2.3(5), we see that $g$ and $F(c)$ must be consecutive on $f$. Hence, there is $f_{1} \in F\left(c_{1}\right)$, with $\left|f_{1}\right|=5$, so that $g$ and $f_{1}$ are consecutive on $f$. By the definition of $\Pi_{f}$, and 2.3(0), we deduce that $f_{1}$ is adjacent to exactly one 3 -face of $G$ (which is in $\left.F\left(c_{1}\right)\right) . g \in F\left(c_{2}\right)$ being a 9-face consecutive on $f$ with the 5 -face $f_{1}$ and the assumption that $t\left(c_{2}\right) \notin T_{1}$, imply that $t\left(c_{2}\right) \in\left\{\left(5,9,3,5^{3}\right),\left(9,3,5^{3}\right),(9,3,6)\right\}$. Let $f_{2} \in F\left(c_{1}\right)$ such that $\left|f_{2}\right|=3$ and $f_{1}$ and $f_{2}$ are consecutive on $f$. Note that it is possible that $f_{1} \in F\left(c_{2}\right)$. Still we easily see that $c_{1} \cup f_{1} \cup f_{2} \subseteq G$ contains a 16-cycle, a contradiction.

Case 3. Suppose $t\left(c_{1}\right)=\left(9,3,5^{3}\right)$. As in Case (1), we first see that (3.i) $c_{1}$ is disjoint from $f^{\prime}$, for any $f^{\prime} \in F(G) \backslash F\left(c_{1}\right)$ with $\left|f^{\prime}\right| \in\{6,9\}$.

Let $F\left(c_{1}\right)=\left\{f_{1}, \ldots, f_{4}\right\}$ such that $\left|f_{1}\right|=9,\left|f_{2}\right|=\left|f_{4}\right|=3$ and $\left|f_{3}\right|=5$, and Chain $\left(c_{1}\right)=\left\{f_{1}, f_{2}, f_{3}\right\}$. Let $g \in F\left(c_{2}\right)$ as exists by 3.7. By (i), $|g|=5$ or $|g|=3$.

Case 3.1 Suppose $|g|=5$. If $t\left(c_{2}\right)=(5)$ but $V\left(P\left(c_{1}\right)\right) \cap V\left(P\left(c_{2}\right)\right) \neq \emptyset$, then by 2.3(2), $c^{\prime}:=g \cup c_{1}$ is a cluster of $f, F\left(c_{1}\right) \subseteq F\left(c^{\prime}\right)$ and $t\left(c^{\prime}\right)=\left(5,9,3,5^{3}\right) \in \Im_{\text {all }}$, contradicting the definition of $\Pi_{f}$. Suppose then that $t\left(c_{2}\right) \neq(5)$. We may assume that $t\left(c_{2}\right) \neq\left(5,9,3,5^{3}\right)$, for otherwise it can be verified that $c^{\prime}:=c_{1} \cup c_{2}$ a cluster of $f, F\left(c_{1}\right) \subseteq F\left(c^{\prime}\right)$ and $t\left(c^{\prime}\right) \in$ $\left\{\left(5,9,3,5^{3}\right),\left(5^{3}, 3,9,5,9,3,5^{3}\right)\right\} \subseteq \Im_{\text {all }}$, contradicting the definition of $\Pi_{f}$. Hence, by inspection of $\Im_{\text {all }}$, there is $g_{1} \in F\left(c_{2}\right)$ so that $g$ and $g_{1}$ are adjacent, $g_{1}$ and $f$ are adjacent, and $g_{1} \in\{3,6\}$. If $\left|g_{1}\right|=6$, then by (3.i), $g_{1}$ and $c_{1}$ are disjoint; but since $g$ is a 5-face, then by 2.3(4), $V(g) \cap V\left(c_{1}\right)=V(g) \cap V\left(f_{1}\right)$, and the faces $g, f_{1}$ and $f_{3}$ contradict 2.2(1). If $\left|g_{1}\right|=3$, then by 2.3 and 2.2(1), $g_{1}$ and $f_{1}$ are adjacent, and then also consecutive. But then $c^{\prime}:=c_{1} \cup g_{1}$ a cluster of $f, F\left(c_{1}\right) \subseteq F\left(c^{\prime}\right)$ and $t\left(c^{\prime}\right)=\left(3,9,3,5^{3}\right) \in \Im_{\text {all }}$, contradicting the definition of $\Pi_{f}$. 
Case 3.2 Suppose $|g|=3$. By the same arguments as in previous cases, we may assume that $g$ is not adjacent to $f$, and that $g$ is not adjacent to a 6 -face.

We conclude that $t\left(c_{2}\right) \in \Im_{C}^{9}$. By Cases $(1,2)$ and the definition of $\Im_{C}^{9}$, it follows that $t\left(c_{2}\right)=\left(9,3,5^{3}\right)$. Then, it is easily seen that $c^{\prime}:=c_{1} \cup c_{2}$ is a cluster of $f, F\left(c_{1}\right) \subseteq F\left(c^{\prime}\right)$ and $t\left(c^{\prime}\right)=\left(5^{3}, 3,9,3,5^{3}\right) \in \Im_{\text {all }}$ contradicting the definition of $\Pi_{f}$.

Case 4. Suppose $t\left(c_{1}\right) \in\{(3,9,3,5),(9,3,6),(3,9,3,6)\}$. The proof in this case follows by similar arguments as in Cases $(1,3)$.

Case 5. Suppose $t\left(c_{1}\right)=\left(5,9,3,5^{3}\right)$ and that $V\left(c_{2}\right) \cap V\left(c^{\prime}\right) \neq \emptyset$, or that $V\left(P\left(c_{1}\right)\right) \cap$ $V\left(P\left(c_{2}\right)\right) \neq \emptyset$. Let $F\left(c_{1}\right)=\left\{f_{1}, \ldots, f_{5}\right\}$ such that $\left|f_{1}\right|=\left|f_{4}\right|=5,\left|f_{2}\right|=9$ and $\left|f_{3}\right|=$ $\left|f_{5}\right|=3$ and Chain $\left(c_{1}\right)=\left\{f_{1}, f_{2}, f_{3}, f_{4}\right\}$.

If $V\left(c_{2}\right) \cap V\left(c^{\prime}\right) \neq \emptyset$, then let $g \in F\left(c_{2}\right) \backslash F\left(c_{1}\right)$, so that $V(g) \cap V\left(c_{1}\right) \neq \emptyset$. But then it is easily seen that $c_{1} \cup g$ contains a 16-cycle. Hence, $V\left(c_{2}\right) \cap V\left(c^{\prime}\right)=\emptyset$. If $V\left(P\left(c_{1}\right)\right) \cap V\left(P\left(c_{2}\right)\right) \neq \emptyset$, then there is (as in Case (2)) $g \in F\left(c_{2}\right) \backslash F\left(c_{1}\right)$, so that $g$ and $f_{1}$ are consecutive on $f$. But then it is only possible that $|g|=9$, for otherwise we can find a 16-cycle in $c_{1} \cup g \subseteq G$. Now since $c_{2}$ contains a 9-face, by 3.9 and Cases $(1,4)$, $t\left(c_{2}\right)=\left(5,9,3,5^{3}\right)$. But then we deduce that $c:=c_{1} \cup c_{2}$ is a cluster of $f$ of type $\left(5^{3}, 3,9,5,9,3,5^{3}\right)$ containing $c_{1}$, contradicting the definition of $\Pi_{f}$.

The following is verified by inspection.

3.12. If $c_{1}, c_{2} \in \Pi_{f}$ are distinct and $t\left(c_{1}\right), t\left(c_{2}\right) \in \Im_{S} \cup \Im_{C}^{6}$, then Chain $\left(c_{1}\right) \neq$ Chain $\left(c_{2}\right)$.

3.13. Under Hypothesis $A$, let $c_{1}, c_{2} \in \Pi_{f}$ be distinct and suppose that $t\left(c_{1}\right) \in \Im_{C}^{6}$.

(i) Suppose that $t\left(c_{1}\right)=\left(6^{6} 6\right)$. Then, Chain $\left(c_{1}\right)$ and $c_{2}$ are disjoint.

(ii) If $t\left(c_{1}\right) \in \Im_{C}^{6} \backslash\left\{\left(6^{3} 6\right)\right\}$, then $c_{1}$ and $c_{2}$ are disjoint.

Proof. (i) It suffices to show that $P\left(c_{1}\right)$ and $P\left(c_{2}\right)$ are disjoint (the proof then follows by the same arguments as presented in the proofs of 2.10 and 2.9).

We may assume that $t\left(c_{2}\right) \notin\left(\Im_{C}^{3} \cup \Im^{9} \cup \Im_{C}^{5} \cup \Im_{C}^{6^{*}}\right)$, for otherwise the claim follows by $3.11,3.8,3.9$, and 3.10 .

Now, assume for a contradiction that $V\left(P\left(c_{1}\right)\right) \cap V\left(P\left(c_{2}\right)\right) \neq \emptyset$. By 3.12, Chain $\left(c_{1}\right) \neq$ Chain $\left(c_{2}\right)$. Hence that there is $g \in$ Chain $\left(c_{2}\right)$ such that $g \notin F\left(c_{1}\right)$ and $V(g) \cap V\left(P\left(c_{1}\right)\right) \neq \emptyset$. By inspection of $t\left(c_{2}\right)$, we see that $|g| \in\{3,5,6\}$. By 2.3(0), we may also assume that $g$ and Chain $\left(c_{1}\right)$ are consecutive of $f$.

By symmetry we may assume that $E(g) \cap E(f)=\left\{x_{k} x_{1}\right\}$ (the case in which $E(g) \cap$ $E(f)=\left\{x_{\ell+1} x_{\ell+2}\right\}$ is symmetric due to the symmetry of $\left.c_{1}\right)$. Let $F\left(c_{1}\right)=\left\{f_{1}, \ldots, f_{4}\right\}$ such that Chain $\left(c_{1}\right)=\left\{f_{1}, f_{2}\right\}$, and $\left|f_{3}\right|=3$. By $2.3(3),|g| \neq 3$.

We may assume that $|g| \neq 5$, for otherwise by $2.5, c^{\prime}=g \cup c_{1}$ is a cluster of $f$ of type $\left(5,6^{6} 6\right)$ such that $F\left(c_{1}\right) \subseteq F\left(c^{\prime}\right)$, contradicting the definition of $\Pi_{f}$.

Hence $|g|=6$. As $t\left(c_{2}\right) \in \Im_{\text {all }} \backslash\left(\Im_{C}^{3} \cup \Im^{9} \cup \Im_{C}^{5} \cup \Im_{C}^{6^{*}}\right)$, by inspection of $\Im_{\text {all }}$, there is a 3-face, $g_{1} \in F\left(c_{2}\right)$, such that $g$ and $g_{1}$ are adjacent.

If $g_{1}$ is adjacent to $f$, then $E(f) \cap E\left(g_{1}\right)=\left\{x_{k-1} x_{k}\right\}$. By 2.5 and 2.3(3), $c^{\prime}=g \cup g_{1} \cup c_{1}$, is a cluster of $f$ of type $\left(3,6,6^{6} 6\right)$ with $F\left(c_{1}\right) \subseteq F\left(c^{\prime}\right)$, contradicting the definition of $\Pi_{f}$.

If $g_{1}$ is not adjacent to $f$, then as $g \notin F\left(c_{1}\right), g_{1} \neq f_{3}$. By inspection of $t\left(c_{2}\right)$, we see that $t\left(c_{2}\right) \in \Im_{C}^{6}$, and thus there is a 6-face, $g_{2} \in F\left(c_{2}\right)$, such that $g, g_{1}$ and $g_{2}$ are pairwise 
adjacent and $g_{2}$ is adjacent to $f$. As $g_{1} \neq f_{3}$, then by $2.3(3), g_{2} \neq f_{1}$. But then, using 2.5 and 1.3, we can easily verify that $f_{1} \cup f_{2} \cup f_{3} \cup g \cup g_{1} \cup g_{2} \subseteq$ contains a 4-, 8- or 16-cycle; a contradiction.

(ii) Suppose that $t\left(c_{1}\right) \in \Im_{C}^{6} \backslash\left\{\left(6^{3} 6\right)\right\}$, and assume for a contradiction that $V\left(c_{1}\right) \cap V\left(c_{2}\right) \neq$ $\emptyset$. We assume that $t\left(c_{1}\right)=\left\{\left(6^{3} 6,5\right)\right\}$, for if $t\left(c_{1}\right) \in\left\{\left(6^{3} 6,5,3\right),\left(6^{6} 6,6,3\right)\right\}$ then proof follows by similar arguments. Let $\left\{f_{1}, \ldots, f_{5}\right\}=F\left(c_{1}\right)$ such that Chain $\left(c_{1}\right)=\left\{f_{1}, f_{2}, f_{3}\right\}$, $\left|f_{1}\right|=\left|f_{2}\right|=\left|f_{5}\right|=6,\left|f_{3}\right|=5$ and $\left|f_{4}\right|=3$. Let $g$ be as exists by 3.7. By 2.9(i) and 2.8, $|g| \notin\{5,6,9\}$. Hence $|g|=3$. By 2.3(4), $V(g) \cap V\left(c_{1}\right) \subseteq V\left(f_{3}\right) \backslash V\left(f_{2}\right)$. Now, if $g$ is adjacent to $f$, then $c^{\prime}=g \cup c_{1}$ is a cluster of $f$ of type $\left(6^{3} 6,5,3\right)$, and $F\left(c_{1}\right) \subseteq F\left(c^{\prime}\right)$, contradicting the definition of $\Pi_{f}$. If $g$ is not adjacent to $f$, we see that $t\left(c_{2}\right) \in \Im_{C}^{9} \cup \Im_{C}^{3}$, contradicting the definition of $c_{2}$.

We can now turn to the proof of 3.4 .

Proof of 3.4. (1) For suppose not. By 3.8, 3.9, 3.10, 3.11 and 3.13, $t\left(c_{1}\right), t\left(c_{2}\right) \notin \Im_{C}^{3} \cup$ $\Im_{C}^{9} \cup \Im_{C}^{6} \cup \Im_{C}^{5} \cup \Im_{C}^{6^{*}}$. Hence,

$t\left(c_{1}\right), t\left(c_{2}\right) \in \Im_{S} \backslash\{(3,9,3,5),(5,3,9,3,5),(9,3,5,3),(3,9,3,5,3),(9,3,6),(3,9,6,3)\}$.

Let $F\left(c_{1}\right)=\left\{f_{1}, \ldots, f_{\ell}\right\}$, and assume, without loss of generality, that $E(f) \cap E\left(f_{i}\right)=$ $\left\{x_{i} x_{i+1}\right\}$, for $1 \leqslant i \leqslant \ell$. By symmetry, assume that if $c_{1}$ is of type $\left(a_{1}, a_{2}, \ldots, a_{\ell}\right)$, then $\left|f_{i}\right|=a_{i}, 1 \leqslant i \leqslant \ell$.

As $c_{1}$ and $c_{2}$ are distinct, then by 3.12, Chain $\left(c_{1}\right) \neq$ Chain $\left(c_{2}\right)$. Hence, there is $g \in$ Chain $\left(c_{2}\right)$ such that $g \notin F\left(c_{1}\right)$ and $V(g) \cap V\left(P\left(c_{1}\right)\right) \neq \emptyset$. In particular, $g$ and the faces of $F\left(c_{1}\right)$ are consecutive of $f$ (and then $E(g) \cap E(f)=\left\{x_{\ell+1} x_{\ell+2}\right\}$ or $E(g) \cap E(f)=\left\{x_{k} x_{1}\right\}$ ). By inspection of $t\left(c_{2}\right),|g| \in\{3,5,6\}$.

Case 1. Suppose that $t\left(c_{1}\right) \in\{(3,6,6,3,6,6,3),(3,6,5,6,3),(3,6,6,3)\}$. By the symmetry of $c_{1}$ we may assume that $E(g) \cap E(f)=\left\{x_{k} x_{1}\right\}$. By $2.3(1)$ and $(2),|g| \notin$ $\{3,5\}$. Hence, $|g|=6$. If $t\left(c_{1}\right)=(3,6,6,3,6,6,3)$, then $g, f_{1}, \ldots, f_{5}$ are consecutive on $f$, of lengths, $6,3,6,6,3,6$, respectively. If $t\left(c_{1}\right)=(3,6,5,6,3)$, then $g, f_{1}, \ldots, f_{4}$ are consecutive on $f$, of lengths, 6, 3,6,5,6, respectively. Both cases contradict 3.3.

If $t\left(c_{1}\right)=(3,6,6,3)$, then by $3.2(2)$ and $3.1, c^{\prime}=g \cup f_{1} \cup, \ldots, \cup f_{4}$, is a cluster of $f$ of type $(6,3,6,6,3)$, with $F\left(c_{1}\right) \subseteq F\left(c^{\prime}\right)$; contradicting the definition of $\Pi_{f}$.

Case 2. Suppose that $t\left(c_{1}\right)=(6,3,6,6,3)$. If $E(g) \cap E(f)=\left\{x_{\ell+1} x_{\ell+2}\right\}$, then by 2.3 (1) and (2), $|f| \notin\{3,5\}$, and by $3.3,|g| \neq 6$. Hence $E(g) \cap E(f)=\left\{x_{k} x_{1}\right\}$. By 2.3 (3), $|g| \neq 3$, and by 3.3, $|g| \neq 5$. Hence, $|g|=6$. By the definition of $c_{2}$, there is $g_{1} \in F\left(c_{2}\right)$ such that $g, g_{1}$ and $f$ are pairwise adjacent. Clearly, $g_{1} \neq f_{2}$. Thus $E(f) \cap E\left(g_{1}\right)=\left\{x_{k-1} x_{k}\right\}$, and $g_{1}, g, f_{1}, \ldots, f_{\ell}$ are consecutive on $f$, of lengths $3,6,6,3,6,6,3$, respectively. By 3.2, the union of this faces is a cluster, $c^{\prime}$, of $f$ of type $(3,6,6,3,6,6,3)$, with $F\left(c_{1}\right) \subseteq F\left(c^{\prime}\right)$; contradicting the definition of $\Pi_{f}$.

Case 3. Suppose $t\left(c_{1}\right)=(6,3,6,5,3)$. By $2.3(1),(2)$, (4), and as $|g| \in\{3,5,6\}$, we see that $E(g) \cap E(f) \neq\left\{x_{\ell+1} x_{\ell+2}\right\}$. Hence, $E(g) \cap E(f)=\left\{x_{k} x_{1}\right\}$. By $2.3(3),|g| \neq 3$. If $|g|=5(|g|=6)$, then $g, f_{1}, \ldots, f_{4}$ are consecutive on $f$ of lengths $5,6,3,6,5(6,6,3,6,5)$, respectively; contradicting 3.3 . 
Case 4. If $t\left(c_{1}\right) \in\{(3),(5),(3,5),(3,5,3),(3,6),(3,6,5),(6,3,6),(3,5,6,3)$, $(6,3,6,5)\}$, the proof follows by the same arguments as in Cases (1)-(3). This proves (1).

$(2,3)$ First note that by definition $(5),\left(6^{3} 6\right),\left(6^{3} 6\right) \notin \Im_{P}, \Im_{F}$. Hence, if $V\left(c_{1}\right) \cap V\left(c_{2}\right) \neq \emptyset$, then by $3.8,3.10,3.11$ and 3.13 , we see that:

$$
\begin{gathered}
t\left(c_{1}\right), t\left(c_{2}\right) \in \Im:=\Im_{S} \backslash\{(5),(3,9,3,5),(5,3,9,3,5),(9,3,5,3), \\
(3,9,3,5,3),(9,3,6),(3,9,6,3)\}
\end{gathered}
$$

(2) Suppose not. Let $g_{1} \in F\left(c_{1}\right)$ and $g_{2} \in F\left(c_{2}\right)$ such that $V\left(g_{1}\right) \cap V\left(g_{2}\right) \neq \emptyset$. By inspection of $t\left(c_{1}\right)$ and $t\left(c_{2}\right)$, there are sub-clusters $c_{2}^{\prime}$ of $c_{2}$ and $c_{1}^{\prime}$ of $c_{1}$ (possibly $c_{1}^{\prime}=c_{1}$ or $\left.c_{2}^{\prime}=c_{2}\right)$ such that $g_{1} \in F\left(c_{1}^{\prime}\right), g_{2} \in F\left(c_{2}^{\prime}\right)$, and $t\left(c_{1}^{\prime}\right), t\left(c_{2}^{\prime}\right) \in\{(3,5),(3,6),(3,6,5)\}$. By definition of $g_{1}$ and $g_{2}, V\left(c_{1}^{\prime}\right) \cap V\left(c_{2}^{\prime}\right) \neq \emptyset$; a contradiction to 3.5 .

(3) Suppose not. Then (by the remark above) $t\left(c_{1}\right), t\left(c_{2}\right) \in \Im \cap \Im_{P}$. Let $g \in F\left(c_{2}\right)$ such that $g \notin F\left(c_{1}\right)$ and $V(g) \cap V\left(c_{1}\right) \neq \emptyset$ ( $g$ exists by definition of $\left.\Pi_{f}\right)$. As $t\left(c_{1}\right), t\left(c_{2}\right) \in \Im_{S}$, $|g| \in\{3,5,6\}$. By the definition of $\Im_{S}$, every face in $F\left(c_{1}\right)$ or $F\left(c_{2}\right)$ is adjacent to $f$. Recall that by 3.4(1), $P\left(c_{1}\right) \cap P\left(c_{2}\right)=\emptyset$. This implies that $F\left(c_{1}\right) \cap F\left(c_{2}\right)=\emptyset$ and that $|g| \neq 3$ (and then $t\left(c_{2}\right) \neq(3)$ ). We may also assume that $c_{1} \neq(3)$.

Case 1. Suppose $t\left(c_{1}\right) \in\{(3,5),(3,5,3)\}$. By 2.3(2), $|g| \neq 5$, hence $|g|=6$. By inspection of $t\left(c_{2}\right)$, there is a sub-cluster $c_{2}^{\prime}$ of $c_{2}$ such that $g \in F\left(c_{2}^{\prime}\right)$ and $t\left(c_{2}^{\prime}\right)=(3,6)$. As $V(g) \cap V\left(c_{1}\right) \neq \emptyset$, there is a sub-cluster $c_{1}^{\prime}$ of $c_{1}$ such that $t\left(c_{1}^{\prime}\right)=(3,5)$ and $V(g) \cap V\left(c_{1}^{\prime}\right) \neq$ $\emptyset$. But then $V\left(c_{1}^{\prime}\right) \cap V\left(c_{2}^{\prime}\right) \neq \emptyset$ and $P\left(c_{1}^{\prime}\right)$ and $P\left(c_{2}^{\prime}\right)$ are disjoint (as $P\left(c_{1}\right)$ and $P\left(c_{2}\right)$ are disjoint); contradicting 3.5(1).

Case 2. Suppose $t\left(c_{1}\right) \in\{(3,6,5,6,3),(3,6,5,3)\}$. By inspection of $t\left(c_{2}\right)$, there is a sub-cluster $c_{2}^{\prime}$ of $c_{2}$ such that $g \in F\left(c_{2}^{\prime}\right)$ and $t\left(c_{2}^{\prime}\right) \in\{(3,5),(3,6)\}$. Hence, $V\left(c_{1}\right) \cap V\left(c_{2}^{\prime}\right) \neq$ $\emptyset$ and $V\left(P\left(c_{1}\right)\right) \cap V\left(P\left(c_{2}^{\prime}\right)\right)=\emptyset$; contradicting 3.6(3).

Case 3. Suppose $t\left(c_{1}\right) \in\{(6,3,6,5),(6,3,6,5,3)\}$. By Cases $(1,2)$ :

$$
t\left(c_{2}\right) \in\{(6,3,6),(6,3,6,5),(3,6,6,3),(6,3,6,5,3),(6,3,6,6,3),(3,6,6,3,6,6,3)\}
$$

By inspection of $t\left(c_{2}\right)$, there is a sub-cluster of $c_{2}^{\prime}$ of $c_{2}$ such that $g \in F\left(c_{2}^{\prime}\right)$ and if $|g|=6(|g|=5)$, then $t\left(c_{2}^{\prime}\right)=(3,6)\left(t\left(c_{2}^{\prime}\right)=(3,6,5)\right)$. As $V(g) \cap V\left(c_{1}\right) \neq \emptyset$, there is a sub-cluster of $c_{1}, c_{1}^{\prime}$ such that $t\left(c_{1}^{\prime}\right)=(6,3,6,5)$, and $V(g) \cap V\left(c_{1}^{\prime}\right) \neq \emptyset$. Hence, $V\left(c_{1}^{\prime}\right) \cap V\left(c_{2}^{\prime}\right) \neq \emptyset$ and $V\left(P\left(c_{1}^{\prime}\right)\right) \cap V\left(P\left(c_{2}^{\prime}\right)\right)=\emptyset$, contradicting 3.6(1).

Case 4. Suppose $t\left(c_{1}\right) \in\{(3,6,6,3),(3,6,6,3,6,6,3)\}$. By Cases (1-3):

$$
t\left(c_{2}\right)=\{(6,3,6),(3,6,6,3)(6,3,6,6,3),(3,6,6,3,6,6,3)\}
$$

By inspection of $t\left(c_{2}\right)$, there is a sub-cluster $c_{2}^{\prime}$ of $c_{2}$ such that $g \in F\left(c_{2}^{\prime}\right)$ and $t\left(c_{2}^{\prime}\right) \in$ $\{(6,3,6),(3,6,6,3)\}$. As $V\left(c_{1}\right) \cap V(g) \neq \emptyset$, then $V\left(c_{1}\right) \cap V\left(c_{2}^{\prime}\right) \neq \emptyset$, and $V\left(P\left(c_{1}\right)\right) \cap$ $V\left(P\left(c_{2}^{\prime}\right)\right)=\emptyset$; contradicting $3.6(2)$.

Case 5. Suppose $t\left(c_{1}\right)=(6,3,6,6,3)$. By Cases $(1-4), t\left(c_{2}\right) \in\{(6,3,6),(6,3,6,6,3)\}$. By inspection of $t\left(c_{2}\right)$, there is a sub-cluster of $c_{2}^{\prime}$ of $c_{2}$ such that $g \in F\left(c_{2}^{\prime}\right)$, and $t\left(c_{2}^{\prime}\right) \in$ 
$\{(6,3,6),(3,6,6,3)\}$. As $V(g) \cap V\left(c_{1}\right) \neq \emptyset$, then $V\left(c_{1}\right) \cap V\left(c_{2}^{\prime}\right) \neq \emptyset$ and $V\left(P\left(c_{1}\right) \cap\right.$ $V\left(P\left(c_{2}^{\prime}\right)=\emptyset\right.$; contradicting 3.6(4).

(4) The proof follows from 3.13 and 3.9.

(5) The proof follows from 1.3 and 2.2(1).

We conclude this section with following lemma, which can be verified using 1.3 and 2.3(0). The "pseudoclusters" $(3,9)$ and $(3,10)$ that appear in the lemma are defined in a natural way. (However, they do not belong to $\Im_{\text {all }}$.)

3.14. Let $c_{1}, c_{2}$ be distinct clusters of $f$ such that $c_{2} \in \Pi_{f}$ and $F\left(c_{1}\right) \nsubseteq F\left(c_{2}\right)$

1. Suppose $t\left(c_{1}\right)=(3,9)$ and $t\left(c_{2}\right) \in\left\{\left(3,5^{3}\right),(3,5,6,3),(6,3,6),\left(3,9,3,5^{3}\right)\right\}$. Then $c_{1}$ and $c_{2}$ are disjoint.

2. Suppose $t\left(c_{1}\right)=(3,10)$ and $t\left(c_{2}\right)=(6,3,6)$. Then $c_{1}$ and $c_{2}$ are disjoint.

\section{Extending a Cycle}

We start with some definitions. For a subgraph $H \subseteq G$, let $\Delta_{H} \subseteq \mathbb{N}^{*}$ be the set of all lengths of cycles of $H$, that is $x \in \Delta_{H}$ if and only if $H$ contains a cycle of length $x$. $\left(\mathbb{N}^{*}\right.$ is the set of non-negative integers.) For integers $x$ and $y$, with $x \leqslant y$, let $[x, y]=$ $\{x, x+1, \ldots, y\}$.

Let $c \in \Pi_{f}$, for some $f \in F(G)$ and let $S=c \cup f$. Using $c$, the face $f$ can be extended (inside $S$ ) into cycles of greater length than $|f|$. The fact that $G$ contains no $2^{m}$-cycles $(2 \leqslant m \leqslant 7)$ will allow us to characterize the set $\Pi_{f}$. Intuitively, $\Pi_{f}$ will be "small", for otherwise as the clusters in $\Pi_{f}$ are essentially pairwise disjoint, we will be able to extend $f$ into a cycle of length $2^{m}$. Below a set $\Omega_{S} \in \mathbb{N}^{*}$ is defined so that $|f|+\Omega_{S} \subseteq \Delta_{S}$ (that is, for every $\left.\omega \in \Omega_{S},|f|+\omega \in \Delta_{S}\right)$.

4.1. Under Hypothesis $A$, let $c \in \Pi_{f}$ and $S=f \cup c$. Then,

1. If $t(c)=(3)$, set $\Omega_{S}:=\{0,1\}$.

2. If $t(c)=(5)$, set $\Omega_{S}:=\{0,3\}$.

3. If $t(c) \in\{(3,5),(3,5,3)\}$, set $\Omega_{S}:=[0,3]$.

4. If $t(c) \in\{(3,6),(6,3,6)\}$, set $\Omega_{S}:=[0,4] \backslash\{2\}$.

5. If $t(c)=\left\{\left(3,5^{3}\right)\right\}$, set $\Omega_{S}:=[0,4]$.

6. If $t(c) \in\{(3,5,6,3),(3,6,5)\}$, set $\Omega_{S}:=[0,5] \backslash\{2\}$.

7. If $t(c)=(6,3,6,5)$, set $\Omega_{S}:=[0,6] \backslash\{2\}$.

8. If $t(c)=(3,6,6,3)$, set $\Omega_{S}:=[0,6]$.

9. If $t(c) \in\left\{\left(6^{3} 6\right),\left(6^{3} 6,5\right),\left(6^{3} 6\right)\right\}$, set $\Omega_{S}:=\{0,6\}$.

10. If $t(c) \in\left\{(6,3,6,5,3),(3,9,3,5),(5,3,9,3,5),(9,3,6),(3,9,3,6),\left(5,9,3,5^{3}\right)\right\}$, 
11. set $\Omega_{S}:=[0,7] \backslash\{2,5\}$.

12. If $t(c) \in\left\{(9,3,5,3),(3,9,3,5,3),\left(9,3,5^{3}\right),\left(3,9,3,5^{3}\right),\left(5,3,9,3,5^{3}\right),(3,6,5,6,3)\right.$,

$$
\left.\left(6^{3} 6,5,3\right),(6,3,6,6,3),\left(9,5^{3}, 3\right)\right\}
$$

set $\Omega_{S}:=[0,8] \backslash\{5\}$.

13. If $t(c) \in\left\{\left(3,9,5^{3}, 3\right),\left(5^{3}, 3,9,3,5^{3}\right),\left(6^{3} 6,6,3\right)\right\}$, set $\Omega_{S}:=[0,9] \backslash\{2\}$.

14. If $t(c)=(3,6,6,3,6,6,3)$, set $\Omega_{S}:=[0,10]$.

15. If $t(c)=\left(5^{3}, 3,9,5,9,3,5^{3}\right)$, set $\Omega_{S}:=[0,14]$.

16. If $t(c) \in\left\{\left(6^{656} 6\right),\left(6^{6} 5^{6} 6\right)\right\}$, set $\Omega_{S}:=\{0\}$.

The reader may verify by inspection using Figures 2 and 3, that in $4.1, \Omega_{S}$ is indeed always a subset of $\Delta_{S}$.

Let $f, c$, and $S$ be as in 4.1. We say that $c$ extends $f$ by at $\operatorname{most} \max \left(\Omega_{S}\right)$. The value $\max \left(\Omega_{S}\right)$ is called the maximal extension value of $c$ with respect to $f$. As this value is determined in 4.1 solely by the type of $c$, then for $X \in \Im_{\text {all }}$, let

$$
M_{X} \text { be the maximal extension value of a cluster of type } X
$$

The following lemma is straightforward, and can be proved by a simple inductive argument.

4.2. Let $x_{1}, \ldots, x_{n} \in \mathbb{N}^{*}$ such that $1 \leqslant x_{i} \leqslant 14$ and $x_{i} \neq 2(i=1, \ldots, n)$. Let $y_{1}, \ldots, y_{m} \in$ $\mathbb{N}^{*}$ such that $4 \leqslant y_{i} \leqslant 14(i=1, \ldots, m)$. For $i=1, \ldots, n$, let $X_{i}=\left\{0,1,2, \ldots, x_{i}\right\}$. For $i=1, \ldots, m$, define $Y_{i}$ as follows:

- If $4 \leqslant y_{i} \leqslant 5$, set $Y_{i}=\left\{0,1,2, \ldots, y_{i}\right\} \backslash\{2\}$.

- If $6 \leqslant y_{i} \leqslant 14$, set $Y_{i}=\left\{0,1,2, \ldots, y_{i}\right\} \backslash\{2,5\}$.

Let $A=\left\{a_{1}, \ldots, a_{n}\right\} \subseteq \mathbb{N}^{*}$ and $B=\left\{b_{1}, \ldots, b_{m}\right\} \subseteq \mathbb{N}^{*}$. Let $P(A)=\sum_{i=1}^{n} a_{i}$ and $P(B)=$ $\sum_{i=1}^{m} b_{i}$. Let $r=\sum_{i=1}^{n}\left(a_{i} \cdot x_{i}\right)+\sum_{j=1}^{m}\left(b_{j} \cdot y_{j}\right)$ and $R=\{0,1,2, \ldots, r\}$. If $P(A)+P(B) \geqslant 2$, then each $d \in R$ can be expressed as follows,

$$
d=\sum_{i=1}^{n} \sum_{j=1}^{a_{i}} \hat{x}_{i, j}+\sum_{i=1}^{m} \sum_{j=1}^{b_{i}} \hat{y}_{i, j}, \text { where } \hat{x}_{i, j} \in X_{i}, \hat{y}_{i, j} \in Y_{j}
$$

4.3. Under Hypothesis $A$, let $\Im \subseteq \Im_{F}$, and let $C$ be a set of clusters of $f$ such that the following conditions holds:

1. $|C| \geqslant 2$.

2. for every $c \in C, t(c) \in \Im$. 
3. If $c_{1}, c_{2} \in C$ are distinct, then $c_{1}$ and $c_{2}$ are disjoint, unless $t\left(c_{1}\right), t\left(c_{2}\right)=(6,3,6)$ or $t\left(c_{i}\right)=\left(5,9,3,5^{3}\right)(1 \leqslant i \leqslant 2)$. In the latter case $c_{3-i}$ is disjoint from $c_{i}^{\prime}$, where $c_{i}^{\prime} \subseteq c_{i}$ is the sub-cluster of $c_{i}$ of type $\left(9,3,5^{3}\right)$.

For every $X \in \Im$, let $S_{X}=\{c \in C: t(c)=X\}$. Let $L=\sum_{X \in \Im}\left|S_{X}\right| \cdot M_{X}$, and $G^{*}=f \cup\left(\bigcup_{c \in C} c\right)$. Then $\{|f|,|f|+1, \ldots,|f|+L\} \subseteq \Delta_{G^{*}}$.

Proof. Let $S_{(6,3,6)}=\left\{c_{1}, \ldots, c_{m}\right\} \subseteq C$ and $S_{\left(5,9,3,5^{3}\right)}=\left\{d_{1}, \ldots, d_{\ell}\right\} \subseteq C$ (where $m, \ell \geqslant 0$ ). By 3.4, $c_{i}(i=1, \ldots, m)$ contains a sub-cluster $\hat{c}_{i}$ of type $(3,6)$ such that for distinct $1 \leqslant j, r \leqslant m, \hat{c}_{j}$ and $\hat{c}_{r}$ are disjoint. By assumption, $\hat{c}_{i}$ is disjoint from $c$, for every $c \in C \backslash S_{(6,3,6)}$. By assumption again, $d_{i}(i=1, \ldots, \ell)$ contains a sub-cluster $\hat{d}_{i}$ of type $\left(9,3,5^{3}\right)$ such that $\hat{d}_{i}$ is disjoint from $c$, for every $c \in C \backslash d_{i}$. We conclude that the clusters in the set $C^{\prime}=\left(C \backslash\left(S_{6,3,6} \cup S_{\left(5,9,3,5^{3}\right)}\right)\right) \cup \bigcup_{i=1}^{m} \hat{c}_{i} \cup \bigcup_{i=1}^{\ell} \hat{d}_{i}$ are pairwise disjoint, and for each $c \in C^{\prime}, t(c) \in \Im_{F} \backslash(6,3,6)$.

Let $S_{X}^{\prime}=\left\{c \in C^{\prime}: t(c)=X\right\}$ and let $L^{\prime}=\sum_{X \in \Im}\left|S_{X}^{\prime}\right| \cdot M_{X}$. By 4.1, $M_{(3,6)}=M_{(6,3,6)}$ and $M_{\left(5,9,3,5^{3}\right)}=M_{\left(9,3,5^{3}\right)}$. Hence $L^{\prime}=L$.

For $c \in C^{\prime}$, set $x_{c}=t(c) \in \Im_{F}$ and $H_{c}:=f \cup c$. As $\Im \subseteq \Im_{F}$, by $4.1,1 \leqslant M_{X} \leqslant 14$, and the following holds:

1. If $4 \leqslant M_{X} \leqslant 5$, then $\left\{0,1, \ldots, M_{X}\right\} \backslash\{2\} \subseteq \Omega_{H_{c}}$.

2. If $6 \leqslant M_{X} \leqslant 14$, then $\left\{0,1, \ldots, M_{X}\right\} \backslash\{2,5\} \subseteq \Omega_{H_{c}}$.

The proof follows from 4.2 and the disjointness of the clusters in $C^{\prime}$.

Define a function $\alpha: \mathbb{N} \rightarrow \mathbb{N}$ as follows:

$$
\alpha(x)=2^{\left\lfloor\log _{2} x\right\rfloor+1}
$$

We then have the following straightforward observation.

4.4. In the settings of 4.3, $G^{*}$ contains no $2^{m}$-cycles if and only if $L<\alpha(|f|)-|f|$.

If $c \in \Pi_{f}$ and $t(c) \in\left\{\left(6^{6} 6\right),\left(6^{6} 6,5\right),\left(6^{9} 6\right)\right\}$, then $t(c) \notin \Im_{F}$. Observe that $c$ has a maximal extension value of 6 , however $c$ does not extend $f$ by 1,2 or 3 . This is why these types of clusters are not excluded from the sets $\Im_{F}$ or $\Im_{P}$. Using 3.4(3), the extension values of these clusters are exploited in a different way. The idea is to extend $f$ in steps of 6 as much as possible, thus obtaining (instead of $f$ ) a new cycle which is closer in length to $\alpha(|f|)$ but not exceeding $\alpha(|f|)$.

For $n \geqslant 1$, and a set $\left\{a_{0}, \ldots, a_{n}\right\} \subseteq \mathbb{N}^{*}$, define a function $\beta:\left(a_{0}, \ldots, a_{n}\right) \rightarrow \mathbb{N}$ as follows. $\beta\left(a_{0}, \ldots, a_{n}\right)=m$, where

1. $m \leqslant \sum_{i=1}^{n} a_{i}$

2. $6 \cdot m \leqslant \alpha\left(a_{0}\right)-a_{0}$

3. subject to (1) and (2), $m$ is maximum. 
The following corollary is obtained by applying $4.3,4.4$, and 3.4 to the sets $\Pi_{f}$, $\Im_{P}$ and $\Im_{F}$.

4.5. Under Hypothesis $A$, let $S_{X}=\left\{c \in \Pi_{f}: t(c)=X\right\}$ for every $X \in \Im_{F}$, and let $G^{*}=f \cup\left(\bigcup_{c \in \Pi_{f}} c\right)$.

1. If $\left|\Im_{P}\right| \geqslant 2$, then

$$
\sum_{X \in \Im_{P}}\left|S_{X}\right| \cdot M_{X}<\alpha(|f|)-|f|-\beta\left(|f|,\left|S_{\left(6^{3} 6\right)}\right|,\left|S_{\left(6^{3} 6,5\right)}\right|,\left|S_{\left(6^{3} 6\right)}\right|\right)
$$

2. If $\left|\Im_{F}\right| \geqslant 2$ and $k \in\{17,18\}$, then

$$
\sum_{X \in \Im_{F}}\left|S_{X}\right| \cdot M_{X}<\alpha(|f|)-|f|-\beta\left(|f|,\left|S_{\left(6^{6} 6\right)}^{6}\right|,\left|S_{\left(6^{3} 6,5\right)}^{6}\right|,\left|S_{\left(6^{3} 6\right)}^{9}\right|\right)
$$

\section{Discharging and Integer Programs}

In this section the main theorem is proved using the Discharging Method. The first step in the Discharging Method is to assign numerical values (known as charges) to the elements of $G$. For $v \in V(G)$, let $\operatorname{ch}(v)=4-\operatorname{deg}(v)$ and for $f \in F(G)$, let $\operatorname{ch}(f)=4-|f|$.

The following lemma is a simple consequence of Euler's formula.

5.1. $\sum_{v \in V(G)}(4-\operatorname{deg}(v))+\sum_{f \in F(G)}(4-|f|)=8$.

Our goal is prove that $G$ does not exist. To this end, the charges will be locally redistributed according to Rules(1-15) listed below. This is called discharging, as the rules are designed to send charge away from those elements of positive initial charge. If $x$ is either a vertex or a face of a plane graph, let $\operatorname{ch}^{*}(x)$ (denoted as the modified charge) be the resultant charge after modification of the initial charges of the elements of the graph according to Rules(1-15).

Rule(1). If $f$ is an $(\geqslant 11, \geqslant 11, \geqslant 11)$-face or $(10,10, \geqslant 10)$-face, then $f$ sends $\frac{1}{3}$ to each of the faces adjacent to it.

$\operatorname{Rule}(2)$. If $f$ is a $(10, \geqslant 11, \geqslant 11)$-face, then $f$ sends $\frac{1}{5}$ to the 10 -face adjacent to it, and $\frac{2}{5}$ to each $\geqslant 11$ face adjacent to it.

Rule(3).If $f$ is a $(9, \geqslant 11, \geqslant 11)$-face, then $f$ sends $\frac{1}{2}$ to each $\geqslant 11$ face adjacent to it.

Rule(4). Let $f$ be a $(5, \geqslant 10, \geqslant 10)$-face. Let $\Gamma(f)=\left\{f_{1}, f_{2}, f_{3}\right\}$ such that $\left|f_{1}\right|=5$.

(a) If $f$ is the only 3 -face adjacent to $f_{1}$, then $f$ sends $\frac{1}{2}$ to each $\geqslant 10$ face adjacent to it; otherwise

(b) $f_{1}$ is adjacent to a 3 -face $g$ such that $g \neq f$. If $f_{2}$ and $g$ are disjoint then $f$ sends $\frac{5}{6}$ to $f_{2}$, and $\frac{1}{6}$ to $f_{3}$. If $f_{3}$ and $g$ are disjoint then $f$ sends $\frac{5}{6}$ to $f_{3}$, and $\frac{1}{6}$ to $f_{2}$. 
Rule(5). Let $f$ be a $(5,9, \geqslant 11)$-face. Let $\Gamma(f)=\left\{f_{1}, f_{2}, f_{3}\right\}$ such that $\left|f_{1}\right|=5$ and $\left|f_{2}\right|=9$. Then,

(a) $f$ sends $\frac{1}{6}$ to $f_{2}$ and $\frac{5}{6}$ to $f_{3}$, unless

(b) there exists a 3 -face $g \neq f$ such that $g$ is adjacent to $f_{1}$ and $f_{2}$, and then $f$ sends 1 to $f_{3}$.

Rule(6). Let $f$ be $(6, \geqslant 9, \geqslant 9)$-face.

(a) If $f$ is a $(6,9, \geqslant 11)$-face, then $f$ sends $\frac{1}{6}$ to the adjacent 9 -face, and $\frac{5}{6}$ to the adjacent $\geqslant 11$-face

(b) If $f$ is a $(6, \geqslant 10, \geqslant 10)$-face, then $f$ sends $\frac{1}{2}$ to each adjacent $\geqslant 10$-face.

$\operatorname{Rule}(7)$. Let $f$ be $(6,6, k)$-face such that $k \geqslant 9$. Let $\Gamma(f)=\left\{f_{1}, f_{2}, f_{3}\right\}$ such that $\left|f_{1}\right|=k$.

(a) If $k \geqslant 10$, then $f$ sends $\frac{4}{3}$ to $f_{1}$.

(b) If $k=9$, then let $g$ be the face which is semi-adjacent to $f$ such that $v \in V(f)$, $u \in V(g)$, and $v$ is a $(3,6,6)$-vertex. Then, (i) $f$ sends $\frac{2}{3}$ to $f_{1}$, and (ii) if $|g| \geqslant 7$, then $f$ sends $\frac{2}{3}$ to $g$.

Rule(8). A $(6,6,6)$-face sends 1 to each $\geqslant 9$ semi-adjacent face.

Rule(9). A 5-face not adjacent to 3 -faces but adjacent to at least two $\geqslant 7$-face, sends $\frac{1}{3}$ to each $\geqslant 7$-face face adjacent to it.

Rule(10). A ( $\geqslant 10,6,6,6,6)$-face sends $\frac{2}{3}$ to the $\geqslant 10$ face adjacent to it.

Rule(11). A $(6,6,6,6,6)$-face sends $\frac{1}{6}$ to each $\geqslant 10$ semi-adjacent face.

Rule(12) A $(3, \geqslant 9, \geqslant 9)$-vertex sends $\frac{1}{2}$ to each incident $\geqslant 9$ face.

Rule(13) A $(3, \geqslant 9,6)$-vertex sends $\frac{1}{3}$ to the incident 6 -face, and $\frac{2}{3}$ to the incident $\geqslant 9$-face.

Rule(14). Let $v$ be a $(3,5, \geqslant 9)$-vertex. Then,

(a) $v$ sends 1 to the incident $\geqslant 9$-face, unless

(b) the 5-face incident to $v$ is adjacent to two 3-faces, and every neighbour of $v$ is incident to a 3 -face. In this case $v$ sends $\frac{2}{3}$ to the incident 9 -face and $\frac{1}{3}$ to the incident 5 -face.

Rule(15). A vertex $v$ not sending charge by Rules (12-14), sends $\frac{1}{3}$ to each incident face.

Remark 1. One note for clarification. Suppose $f_{1}, f_{2} \in F(G)$ are two semi-adjacent faces, and let $v \in V\left(f_{1}\right)$ and $u \in V\left(f_{2}\right)$ such that $v u \in E(G)$. If $f_{1}$ sends charge to $f_{2}$, then the charge is sent via $v$ and $u$, i.e., $f_{1}$ sends the charge to $v, v$ sends the charge to $u$, and $u$ sends the charge to $f_{2}$. This enables us to assume that charge enters a face only from the elements $V(f) \cup E(f)$. 
Now the proof of the main theorem may be given. For the graph $G$, it will be shown that every vertex and every face has a non-positive modified charge. The sum of all the modified charges is then non-positive, contradicting 5.1 .

Consider a vertex $v \in V(G)$. Then $\operatorname{ch}(v)=1$. By Rules(12-15), it is easily seen that $v$ sends a total charge of 1 to the faces incident to it. By our rules, if $v$ receives charge, then this is because $v$ is a link between two semi-adjacent faces, and every charge that enters $v$ is sent out of $v$. Hence, $\operatorname{ch}^{*}(v)=0$.

5.2. If $|f| \leqslant 7$, then $\operatorname{ch}^{*}(f) \leqslant 0$.

Proof. Suppose $|\boldsymbol{f}|=3$. Then $\operatorname{ch}(f)=1$.

Let $\Gamma(f)=\left\{f_{1}, f_{2}, f_{3}\right\}$. We may assume that $f$ is adjacent to a $\leqslant 9$-face, for otherwise by $\operatorname{Rules}(1,2,12)$, it is easily seen that $\operatorname{ch}^{*}(f)=0$.

Case 1. Assume that $f$ is adjacent to a 9 -face, say $f_{1}$. If $\left|f_{2}\right|,\left|f_{3}\right| \geqslant 9$, then by $2.2(1,2)$, $\left|f_{2}\right|,\left|f_{3}\right| \geqslant 11$, and $\mathrm{ch}^{*}(f)=0$ by Rule(3,12). Hence, by symmetry, assume that $\left|f_{2}\right| \leqslant 7$. By 2.2(1) and 2.3(0), $5 \leqslant\left|f_{2}\right| \leqslant 6$. If $\left|f_{2}\right|=5$, then by $2.2(1,2)$ and $2.3(0),\left|f_{3}\right| \geqslant 11$. Thus $f$ is a $(5,9, \geqslant 11)$-face, and $\mathrm{ch}^{*}(f)=0$ by Rule(5a,5b,14). If $\left|f_{2}\right|=6$, then as above, $\left|f_{3}\right| \geqslant 11$ or $\left|f_{3}\right|=6$. If $\left|f_{3}\right| \geqslant 11$, then $\operatorname{ch}^{*}(f)=0$ by Rule(6a,12,13). If $\left|f_{3}\right|=6$, then by $2.11,|g| \geqslant 7$ (where $g$ is as in Rule(7b)). By Rule(15), $f$ receives a charge of $\frac{1}{3}$ from the single $(3,6,6)$-vertex incident to it, and by Rule(7b), $f$ sends $\frac{2}{3}$ to each of $f_{1}$ and $g$. Hence, $\operatorname{ch}^{*}(f)=0$.

Case 2. Suppose $f$ is not adjacent to a 9 -face, but adjacent to a $\leqslant 7$-face, say $f_{1}$. By $2.2(1), 5 \leqslant\left|f_{1}\right| \leqslant 6$. If $\left|f_{1}\right|=5$, then, by $2.2(1)$ and as $f$ is not adjacent to a 9-face, $\left|f_{2}\right|,\left|f_{3}\right| \geqslant 10$, and by Rule $(4,12,14), \operatorname{ch}^{*}(f)=0$. Hence, $\left|f_{1}\right|=6$, and thus for $i=2,3$, $\left|f_{i}\right|=6$ or $\left|f_{i}\right| \geqslant 10$. If $\left|f_{2}\right| \neq 6$ or $\left|f_{3}\right| \neq 6$, then $\operatorname{ch}^{*}(f)=0$ by Rules $(6 \mathrm{~b}, 7 \mathrm{a}, 12,13)$. Otherwise, $f$ is a $(6,6,6)$-face. By $2.7, f$ has at least two semi-adjacent, say $g_{1}$ and $g_{2}$ such that $\left|g_{1}\right|,\left|g_{2}\right| \geqslant 9$. We then see that $\operatorname{ch}^{*}(f)=0$, as by $\operatorname{Rule}(15), f$ receives a charge of $\frac{1}{3}$ from each vertex incident to it, and by Rule(8), $f$ sends 1 to each of $g_{1}$ and $g_{2}$.

Suppose $|\boldsymbol{f}|=\mathbf{5}$. Then $\operatorname{ch}(f)=-1$. Let $x_{1}, \ldots, x_{5}$ be the vertices of $f$ in a cyclic order. Let $\Gamma(f)=\left\{f_{1}, \ldots, f_{5}\right\}$ and assume that $E(f) \cap E\left(f_{i}\right)=x_{i} x_{i+1}$ (where $x_{0}:=x_{5}$ and $\left.x_{6}:=x_{1}\right)$. By our rules, $f$ receives no charge from semi-adjacent faces.

Case 1. Suppose that $f$ is not adjacent to a 3 -face. Then $\left|f_{i}\right| \geqslant 6$, for $i=1, \ldots, 5$. Let $\delta$ be the number of $\geqslant 7$ faces adjacent to $f$. If $\delta \geqslant 2$, then then by $\operatorname{Rules}(9,15)$, $\operatorname{ch}^{*}(f) \leqslant-1+5 \cdot \frac{1}{3}-\delta \cdot \frac{1}{3} \leqslant 0$. If $\delta=1$, say $\left|f_{1}\right| \geqslant 7$, then by $2.14,\left|f_{1}\right| \geqslant 10$, and then by $\operatorname{Rules}(10,15), \operatorname{ch}^{*}(f) \leqslant-1+5 \cdot \frac{1}{3}-\frac{2}{3}=0$. If $\delta=0$ (i.e., $f$ is a $(6,6,6,6,6)$-face), then by 2.15 each semi-adjacent face of $f$ is of length $\geqslant 10$. Hence, by Rules $(11,15)$, $\operatorname{ch}^{*}(f) \leqslant-1+5 \cdot \frac{1}{3}-5 \cdot \frac{1}{6}<0$.

Case 2. Suppose $f$ is adjacent to exactly one 3-face. By symmetry, assume that $\left|f_{1}\right|=3$. By 2.3, $\left|f_{5}\right|,\left|f_{2}\right| \geqslant 9$. Then $\operatorname{ch}^{*}(f)=0$, as by Rule(14a) vertices in $V(f) \cap V\left(f_{1}\right)$ send no charge to $f$, and by Rule(15) every other vertex of $V(f)$ sends $\frac{1}{3}$ to $f$.

Case 3. Suppose $f$ is adjacent to two 3-face. By symmetry and 2.3(1), assume that $\left|f_{1}\right|=\left|f_{3}\right|=3$. By Rule(14a), $x_{4}$ and $x_{1}$ sends no charge to $f$. By Rule(14b) each of $x_{2}$ and $x_{3}$ sends $\frac{1}{3}$ to $f$. By Rule(15), $x_{5}$ sends $\frac{1}{3}$ to $f$. Hence, $\operatorname{ch}^{*}(f)=0$. 
Suppose $|\boldsymbol{f}|=\mathbf{6}$. Then $\operatorname{ch}(f)=-2$. By our Rules, $f$ only receives charge according to $\operatorname{Rules}(13,15)$, and then $\operatorname{ch}^{*}(f)=-2+6 \cdot \frac{1}{3}=0$.

Suppose $|\boldsymbol{f}|=7 \cdot \operatorname{ch}(f)=-3$. Let $\delta$ be the number of 5 -faces adjacent to $f$. By 2.2(1) and $2.3(2), \delta \leqslant 2$.

Case 1. Suppose $f$ receives charge from a semi-adjacent face, say $f^{\prime}$. If this is the case, then Rule(7b) was applied. By this rule, there is a cluster $c$ of $f$ of type $\left(6^{3} 6\right)$ such that $\left|f^{\prime}\right|=3$ and $f^{\prime} \in F(c)$. By this rule, $f^{\prime}$ sends $\frac{2}{3}$ to $f$. By 2.11 and 2.3(0), we see that $\delta=0$ and $f^{\prime}$ is the sole semi-adjacent face sending charge to $f$. Hence, by Rules $(7 \mathrm{~b}, 15)$, $\operatorname{ch}^{*}(f)=-3+7 \cdot \frac{1}{3}+\frac{2}{3}=0$.

Case 2. Suppose $f$ receives no charge from a semi-adjacent face. If $\delta=0$, then $f$ only receives charge by Rule(15), and $\operatorname{ch}^{*}(f)=-3+7 \cdot \frac{1}{3}<0$. If $\delta \geqslant 1$, then let $g$ be a 5 -face adjacent to $f$. We see that $g$ sends to $f$ a charge of at most $\frac{1}{3}$. Indeed, if $g$ is adjacent to a 3 -face, then by our rules $g$ sends no charge to $f$. If $g$ is not adjacent to a 3 -face, then by 2.14 , we conclude that $g$ is adjacent to at least two $\geqslant 7$-faces, and then by Rule(9), $g$ sends $\frac{1}{3}$ to $f$. As $\delta \leqslant 2$, then by Rules $(9,15), \operatorname{ch}^{*}(f) \leqslant-3+7 \cdot \frac{1}{3}+2 \cdot \frac{1}{3}=0$.

Next we have to show that $\operatorname{ch}^{*}(f) \leqslant 0$, for every $f \in F(G)$ with $|f| \geqslant 9$. The proof in this case requires some more elaborate arguments.

\subsection{Under Hypothesis A,}

1. Let $g \in \Gamma(f)$. If $g$ sends charge to $f$, then there is $c \in \Pi_{f}$ with $g \in F(c)$.

2. Let $v \in V(f)$. If $v$ sends charge to $f$ which is strictly larger than $\frac{1}{3}$, then there is $c \in \Pi_{f}$ with $v \in V(P(c))$.

3. Suppose $c \in \Pi_{f}$ and let $v$ be an endpoint of $P(c)$. Let $g \in F(c)$ so that $v \in V(g)$. If $|g| \geqslant 6$, then $v$ sends $\frac{1}{3}$ to $f$.

Proof. (1) By Remark 1 and Rules(1-15), if $g$ sends charge to $f$, then $|g| \in\{3,5\}$ and $g$ is adjacent to $f$. Thus, $c:=g$ is a cluster of $f$ of type $(|g|)$. By definition of $\Pi_{f}$, there is a cluster $c^{\prime}$ of $f$ so that $c \subseteq c^{\prime}$ (possibly $c=c^{\prime}$ ) and $g \in$ Chain $\left(c^{\prime}\right)$, as required.

(2) By Rules(1-15), if $v$ sends to $f$ a charge which is strictly larger than $\frac{1}{3}$, then there is a face $g \in F(G)$ so that that either $|g|=3, g$ and $f$ are adjacent and $v \in V(g) \cap V(f)$ or $|g| \in\{3,5\}, g$ and $f$ are semi-adjacent, and the charge of $g$ is sent to $f$ via $v$. We may assume that latter case holds, as the former follows as in (1). By Rules(7,8,11), g is contained in a cluster of $f$ of type $\left(6^{3} 6\right),\left(6^{3} 6\right)$ or $\left.\left(6^{656} 6\right)\right)$ and the proof follows by definition of $\Pi_{f}$.

(3) Let $g_{1}$ be the face incident to $v$, other than $f$ and $g$. By $3.4(1), g_{1} \notin F\left(c^{\prime}\right)$ for every $c^{\prime} \in \Pi_{f}$ distinct from $c$. In particular, as $\{(3),(5)\} \in \Im_{\text {all }},\left|g_{1}\right| \geqslant 6$. If there exists $g_{2} \in F(c)$ so that $g_{2}$ is semi-adjacent to $f$ and $g_{2}$ sends charge to $f$ via $v$, then $v$ is not an endpoint of $P(c)$. Hence, $v$ is a $(\geqslant 6, \geqslant 9, \geqslant 6)$-vertex which is not a link between $f$ and a semi-adjacent of $f$ which sends charge to $f$. Hence, Rule(15) is applied to $v$.

Let

$$
V_{s}=V(f) \backslash \bigcup_{c \in \Pi_{f}} V(P(c))
$$


and

$$
F_{s}=\Gamma(f) \backslash \bigcup_{c \in \Pi_{f}} F(c)
$$

For $x \in V(f) \cup \Gamma(f)$ denote by $\operatorname{ch}_{f}(x)$ the amount of charge that $x$ sends to $f$ by Rules (1)-(15). Note that by Remark (1), $f$ only receives charge from elements in $V(f) \cup \Gamma(f)$. By Rule(15) and 5.3 we have

$$
\operatorname{ch}_{f}(v)=\frac{1}{3} \text {, for } v \in V_{s}
$$

and

$$
\operatorname{ch}_{f}(g)=0, \text { for } g \in F_{s}
$$

The amount of charge received by $f$ from a cluster $c \in \Pi_{f}$ is then defined as follows:

$$
\operatorname{ch}_{f}(c):=\sum_{v \in V(P(c))} \operatorname{ch}_{f}(v)+\sum_{g \in \operatorname{Chain}(c)} \operatorname{ch}_{f}(g)
$$

Let total $(f)$ denote the total amount of charge received by $f$ from all elements $V(f) \cup$ $\Gamma(f)$. By $3.4(1)$ we have

$$
\operatorname{total}(f)=\sum_{c \in \Pi_{f}} \operatorname{ch}_{f}(c)+\frac{1}{3} \cdot\left|V_{s}\right|
$$

and we conclude that

$$
\operatorname{ch}^{*}(f)=4-|f|+\operatorname{total}(f)=4-|f|+\sum_{c \in \Pi_{f}} \operatorname{ch}_{f}(c)+\frac{1}{3} \cdot\left|V_{s}\right|
$$

Observe that for any $c \in \Pi_{f}, \operatorname{ch}_{f}(c)$ is determined solely by the type of $c$. Now, if we equally spread the total amount of charge that $c$ sends to $f$ among the vertices of $V(P(c))$, then if $v \in V(P(c))$ we may assume that $v$ sends $f$ a charge of

$$
\operatorname{fr}_{f}(c):=\frac{\operatorname{ch}_{f}(c)}{|P(c)|}
$$

Next we provide upper and lower bounds for $\operatorname{ch}_{f}(c)$, where $|f| \geqslant 9$. The following is a direct consequence of Rule(1,15), 5.3 and the structural properties obtained in Section (2).

5.4. Under Hypothesis $A$, let $c \in \Pi_{f}$.

1. Suppose $t(c)=(3)$. Let $F(c)=\left\{f_{1}\right\}$.

(a) If $|f|=9$ then $\operatorname{ch}_{f}(c)=1$ and $\operatorname{fr}_{f}(c)=\frac{1}{2}$.

(b) Suppose $|f| \geqslant 11$.

i. If $f_{1}$ is adjacent to a 9-face, then $\operatorname{ch}_{f}(c)=\frac{3}{2}$ and $\operatorname{fr}_{f}(c)=\frac{3}{4}$.

ii. If $f_{1}$ is adjacent to exactly one 10-face, then $\operatorname{ch}_{f}(c)=\frac{7}{5}$ and $\operatorname{fr}_{f}(c)=\frac{7}{10}$.

iii. If $f_{1}$ is adjacent to two 10-faces, then $\operatorname{ch}_{f}(c)=\frac{4}{3}$ and $\operatorname{fr}_{f}(c)=\frac{2}{3}$.

(c) If $|f|=10$, then $\operatorname{ch}_{f}(c)=\frac{6}{5}$ and $\operatorname{fr}_{f}(c)=\frac{3}{5}$, unless $f_{1}$ is adjacent to at least two 10-faces, and then $\operatorname{ch}_{f}(c)=\frac{4}{3}$ and $\operatorname{fr}_{f}(c)=\frac{2}{3}$. 
2. If $t(c)=(5)$, then $\operatorname{ch}_{f}(c)=1$ and $\operatorname{fr}_{f}(c)=\frac{1}{2}$.

3. Suppose $t(c)=(3,5)$. Let $F_{c}=\left\{f_{1}, f_{2}\right\}$ such that $\left|f_{1}\right|=3$ and $\left|f_{2}\right|=5$.

(a) If $|f|=9$, then $\operatorname{ch}_{f}(c)=2$ and $\operatorname{fr}_{f}(c)=\frac{2}{3}$.

(b) If $|f| \geqslant 10$, then $\operatorname{ch}_{f}(c)=\frac{7}{3}$ and $\mathrm{fr}_{f}(c)=\frac{7}{9}$, unless $f_{1}$ is adjacent to a 9-face, and then $\operatorname{ch}_{f}(c)=\frac{8}{3}$ and $\operatorname{fr}_{f}(c)=\frac{8}{9}$.

4. Suppose $t(c)=(3,5,3)$.

(a) If $|f|=9$, then $\operatorname{ch}_{f}(c)=\frac{7}{3}$ and $\operatorname{fr}_{f}(c)=\frac{7}{12}$.

(b) If $|f| \geqslant 10$, then $\operatorname{ch}_{f}(c)=\frac{8}{3}$ and $\operatorname{fr}_{f}(c)=\frac{2}{3}$.

5. Suppose $t(c)=\left(3,5^{3}\right)$.

(a) If $|f|=9$, then $\operatorname{ch}_{f}(c)=2$ and $\operatorname{fr}_{f}(c)=\frac{2}{3}$.

(b) If $|f| \geqslant 10$, then $\operatorname{ch}_{f}(c)=\frac{8}{3}$ and $\operatorname{fr}_{f}(c)=\frac{8}{9}$.

6. Suppose $t(c)=(3,6)$.

(a) If $|f|=9$ then $\operatorname{ch}_{f}(c)=\frac{5}{3}$ and $\operatorname{fr}_{f}(c)=\frac{5}{9}$.

(b) If $|f| \geqslant 10$, then $\operatorname{ch}_{f}(c)=2$ and $\operatorname{fr}_{f}(c)=\frac{2}{3}$.

7. Suppose $t(c)=(3,6,5)$.

(a) If $|f|=9$, then $\operatorname{ch}_{f}(c)=\frac{7}{3}$ and $\operatorname{fr}_{f}(c)=\frac{7}{12}$.

(b) If $|f| \geqslant 10$, then $\operatorname{ch}_{f}(c)=\frac{8}{3}$ and $\operatorname{fr}_{f}(c)=\frac{2}{3}$.

8. Suppose $t(c)=(6,3,6)$.

(a) If $|f|=9$, then $\operatorname{ch}_{f}(c)=\frac{8}{3}$ and $\operatorname{fr}_{f}(c)=\frac{2}{3}$.

(b) If $|f| \geqslant 10$, then $\operatorname{ch}_{f}(c)=\frac{10}{3}$ and $\operatorname{fr}_{f}(c)=\frac{5}{6}$.

9. If $t(c)=(6,3,6,5)$, then $\operatorname{ch}_{f}(c)=4$ and $\operatorname{fr}_{f}(c)=\frac{4}{5}$.

10. Suppose $t(c)=(3,5,6,3)$.

(a) If $|f|=9$, then $\operatorname{ch}_{f}(c)=\frac{10}{3}$ and $\operatorname{fr}_{f}(c)=\frac{2}{3}$.

(b) If $|f| \geqslant 10$, then $\operatorname{ch}_{f}(c)=4$ and $\operatorname{fr}_{f}(c)=\frac{4}{5}$.

11. If $t(c)=(6,3,6,6,3)$, then $\operatorname{ch}_{f}(c)=5$ and $\operatorname{fr}_{f}(c)=\frac{5}{6}$.

12. If $t(c)=(3,6,6,3,6,6,3)$, then $\operatorname{ch}_{f}(c)=\frac{20}{3}$ and $\operatorname{fr}_{f}(c)=\frac{5}{6}$.

13. Suppose $t(c)=(3,6,6,3)$.

(a) If $|f|=9$, then $\operatorname{ch}_{f}(c)=3$ and $\operatorname{fr}_{f}(c)=\frac{3}{5}$.

(b) If $|f| \geqslant 10$, then $\operatorname{ch}_{f}(c)=\frac{11}{3}$ and $\operatorname{fr}_{f}(c)=\frac{11}{15}$.

14. If $t(c)=(6,3,6,5,3)$, then $\operatorname{ch}_{f}(c)=\frac{16}{3}$ and $\operatorname{fr}_{f}(c)=\frac{8}{9}$.

15. If $c=\left(6^{3} 6\right)$, then $\operatorname{ch}_{f}(c)=2$ and $\operatorname{fr}_{f}(c)=\frac{2}{3}$. 
16. If $t(c)=\left(6^{3} 6\right)$, then $\operatorname{ch}_{f}(c)=\frac{5}{3}$ and $\operatorname{fr}_{f}(c)=\frac{5}{9}$.

17. If $t(c)=\left(6^{3} 6,5\right)$, then $\operatorname{ch}_{f}(c)=\frac{8}{3}$ and $\operatorname{fr}_{f}(c)=\frac{2}{3}$.

18. If $t(c)=\left(6^{3} 6,5,3\right)$, then $\operatorname{ch}_{f}(c)=4$ and $\operatorname{fr}_{f}(c)=\frac{4}{5}$.

19. If $t(c)=\left(6^{3} 6,6,3\right)$, then $\operatorname{ch}_{f}(c)=\frac{11}{3}$ and $\operatorname{fr}_{f}(c)=\frac{11}{15}$.

20. If $t(c)=\left(6^{656} 6\right)$, then $\operatorname{ch}_{f}(c)=\frac{7}{6}$ and $\operatorname{fr}_{f}(c)=\frac{7}{18}$.

21. If $t(c)=\left(6^{6} 5^{6} 6\right)$, then $\operatorname{ch}_{f}(c)=2$ and $\operatorname{fr}_{f}(c)=\frac{1}{2}$.

22. If $t(c)=(9,3,5,3)$, then $\operatorname{ch}_{f}(c)=\frac{11}{3}$ and $\operatorname{fr}_{f}(c)=\frac{11}{15}$.

23. If $t(c)=(3,9,3,5,3)$, then $\operatorname{ch}_{f}(c)=\frac{29}{6}$ and $\operatorname{fr}_{f}(c)=\frac{29}{36}$.

24. If $t(c)=(3,9,3,5)$, then $\operatorname{ch}_{f}(c)=\frac{25}{6}$ and $\operatorname{fr}_{f}(c)=\frac{5}{6}$.

25. If $t(c)=(5,3,9,3,5)$, then $\operatorname{ch}_{f}(c)=\frac{16}{3}$ and $\operatorname{fr}_{f}(c)=\frac{8}{9}$.

26. If $t(c)=\left(9,3,5^{3}\right)$, then $\operatorname{ch}_{f}(c)=\frac{19}{6}$ and $\operatorname{fr}_{f}(c)=\frac{19}{24}$.

27. If $t(c)=\left(3,9,3,5^{3}\right)$, then $\operatorname{ch}_{f}(c)=\frac{13}{3}$ and $\operatorname{fr}_{f}(c)=\frac{13}{15}$.

28. If $t(c)=\left(5,9,3,5^{3}\right)$, then $\operatorname{ch}_{f}(c)=\frac{23}{6}$ and $\operatorname{fr}_{f}(c)=\frac{23}{30}$.

29. If $t(c)=\left(5,3,9,3,5^{3}\right)$, then $\operatorname{ch}_{f}(c)=\frac{11}{2}$ and $\operatorname{fr}_{f}(c)=\frac{11}{12}$.

30. If $t(c)=\left(5^{3}, 3,9,3,5^{3}\right)$, then $\operatorname{ch}_{f}(c)=\frac{17}{3}$ and $\operatorname{fr}_{f}(c)=\frac{17}{18}$.

31. If $t(c)=\left(9,5^{3}, 3\right)$, then $\operatorname{ch}_{f}(c)=3$ and $\operatorname{fr}_{f}(c)=\frac{3}{4}$.

32. If $t(c)=\left(3,9,5^{3}, 3\right)$, then $\operatorname{ch}_{f}(c)=\frac{25}{6}$ and $\operatorname{fr}_{f}(c)=\frac{5}{6}$.

33. If $t(c)=(9,3,6)$, then $\operatorname{ch}_{f}(c)=\frac{8}{3}$ and $\operatorname{fr}_{f}(c)=\frac{2}{3}$.

34. If $t(c)=(3,9,3,6)$, then $\operatorname{ch}_{f}(c)=\frac{23}{6}$ and $\operatorname{fr}_{f}(c)=\frac{23}{30}$.

35. If $t(c)=(3,6,5,6,3)$, then $\operatorname{ch}_{f}(c)=\frac{13}{3}$ and $\operatorname{fr}_{f}(c)=\frac{13}{18}$.

36. If $t(c)=\left(5^{3}, 3,9,5,9,3,5^{3}\right)$, then $\operatorname{ch}_{f}(c)=\frac{20}{3}$ and $\operatorname{fr}_{f}(c)=\frac{5}{6}$.

Proof. As the proof is merely a routine checking, we only prove item (1). Items (2)-(36) are proved in a similar way.

(1) is proved as follows. First note that by the definition of $\Pi_{f}$, every face adjacent to $f_{1}$ is of length at least 9 . If $|f|=9$, then by 2.2 , any face adjacent to $f_{1}$, other than $f$, is of length $\geqslant 11$, and (a) follows by Rules(3,12). Suppose $|f| \geqslant 11$ (the proof when $|f|=10$ follows by the same arguments). If $f_{1}$ is adjacent to a 9 -face, then by 2.2 , the third face adjacent to $f_{1}$ is of size $\geqslant 11$, and the claim follows by $\operatorname{Rules}(3,12)$. If $f_{1}$ is adjacent to exactly one 10 -face, then by $2.2, f_{1}$ is not adjacent to a 9 -face. Thus the third face adjacent to $f_{1}$ is of length $\geqslant 11$ and the claim follows by $\operatorname{Rules}(2,12)$. If $f_{1}$ is adjacent to two 10-faces, then the claim follows by Rules $(2,12)$.

The following shows that $\operatorname{ch}^{*}(f) \leqslant 0$ for all "large" faces. 
5.5. If $|f| \geqslant 72$, then $\operatorname{ch}^{*}(f) \leqslant 0$.

Proof. Let $v \in V(f)$. If $v \in V(P(c))$ for some $c \in \Pi_{f}$, then by (10), $v$ sends a charge of $\operatorname{fr}_{f}(c)$ to $f$. Otherwise, by (5), $v$ sends $\frac{1}{3}$ to $f$. Hence $v$ sends a charge of at most $\mu$ to $f$, where $\mu=\max \left\{\frac{1}{3}, \max _{c \in \Pi_{f}}\left\{\operatorname{fr}_{f}(c)\right\}\right\}$. By $5.4, \mu \leqslant \frac{17}{18}$. Hence, $\operatorname{ch}^{*}(f) \leqslant(4-k)+\frac{17}{18} k$, and for $k \geqslant 72, \operatorname{ch}^{*}(f) \leqslant 0$.

Next we show that $\operatorname{ch}^{*}(f) \leqslant 0$ when $9 \leqslant|f| \leqslant 15$.

5.6. If $9 \leqslant|f| \leqslant 15$, then $\operatorname{ch}^{*}(f) \leqslant 0$.

Proof. Suppose $|\boldsymbol{f}|=15$. By 2.2(1), if $c \in \Pi_{f}$ and $\left|S_{t(c)}\right|>0$, then $F(c)$ contains no 3 -face adjacent to $f$. Hence by inspection of $\Im_{\text {all }}$,

$$
t(c) \in\left\{(5),\left(6^{3} 6\right),\left(6^{3} 6,5\right),\left(6^{656} 6\right),\left(6^{6} 5^{6} 6\right),\left(6^{3} 6\right)\right\}
$$

By $5.4, \operatorname{fr}_{f}(c) \leqslant \frac{2}{3}$. By $(9), \operatorname{ch}^{*}(f) \leqslant 4-|f|+\frac{2}{3} \cdot|f|=4-15+\frac{2}{3} \cdot 15<0$.

Suppose $|\boldsymbol{f}|=\mathbf{1 4}$. If $c \in \Pi_{f}$, then $c$ does not extend $f$ by two. By 4.1

$$
\begin{gathered}
t(c) \in T:=\left\{(3),(5),(3,6),(6,3,6),(3,6,5),(6,3,6,5),\left(6^{3} 6,6,3\right),\left(6^{3} 6\right),\left(6^{3} 6,5\right),\right. \\
\left.\left(6^{656} 6\right),\left(6^{6} 5^{6} 6\right),\left(6^{9} 6\right),(9,3,6)\right\}
\end{gathered}
$$

Let

$$
T^{\prime}:=\left\{(3),(6,3,6),\left(6^{6} 6,6,3\right),(6,3,6,5)\right\} \subseteq T
$$

By 5.4, $\operatorname{fr}_{f}(c) \leqslant \frac{2}{3}$ for every $c \in T \backslash T^{\prime}$. We may assume that $\sum_{X \in T^{\prime}}\left|S_{X}\right| \geqslant 1$; for otherwise $\mathrm{ch}^{*}(f) \leqslant 4-|f|+|f| \cdot \frac{2}{3} \leqslant 4-14+14 \cdot \frac{2}{3}<0$. By 2.3(1), 3.4 (1), and 2.2(1), we deduce that $\sum_{X \in T^{\prime}}\left|S_{X}\right|=1$. Let $X \in T^{\prime}$ so that $\left|S_{X}\right|=1$ and let $c \in S_{X}$. Then, by (9), $\operatorname{ch}^{*}(f) \leqslant 4-|f|+|P(c)| \cdot \mathrm{fr}_{f}(c)+(|f|-|P(c)|) \cdot \frac{2}{3}$. If $X=(6,3,6)$, then by 5.4(8), $\operatorname{fr}_{f}(c) \leqslant \frac{5}{6}$ and $|P(c)|=4$; hence $c^{*}(f) \leqslant 4-14+4 \cdot \frac{5}{6}+10 \cdot \frac{2}{3}=0$. If $X \in T^{\prime} \backslash\{(6,3,6)\}$, then $|P(c)| \leqslant 5$ and by $5.4(1,9,19), \operatorname{fr}_{f}(c) \leqslant \frac{4}{5}$; hence, $\operatorname{ch}^{*}(f) \leqslant 4-14+5 \cdot \frac{4}{5}+9 \cdot \frac{2}{3}=0$.

Suppose $|\boldsymbol{f}|=\mathbf{1 3}$. By $2.3(0), f$ is not adjacent to a 5 -face, and $c$ does not extend $f$ by three, for every $c \in \Pi_{f}$. Hence using 4.1 we see that $\left.t(c) \in\left\{(3),\left(6^{3} 6\right),\left(6^{656} 6\right), 6^{3} 6\right)\right\}$.

By 5.4 , we see that if $\left.t(c) \in\left\{\left(6^{6} 6\right),\left(6^{656} 6\right), 6^{9} 6\right)\right\}$, then $|P(c)|=3$ and $\operatorname{fr}_{f}(c) \leqslant \frac{2}{3}$; and if $t(c)=(3)$, then $|P(c)|=2$ and $\operatorname{fr}_{f}(c) \leqslant \frac{3}{4}$. By $4.5(2),\left|S_{(3)}\right| \leqslant 2$. Hence, $\operatorname{ch}^{*}(f) \leqslant$ $4-|f|+4 \cdot \frac{3}{4}+(|f|-4) \cdot \frac{2}{3}=4-13+4 \cdot \frac{3}{4}+(13-4) \cdot \frac{2}{3}=0$.

Suppose $|\boldsymbol{f}|=\mathbf{1 2}$. By 2.3(0), $f$ is not adjacent to a six face, and $c$ does not extend $f$ by four, for every $c \in \Pi_{f}$. By $4.1, t(c) \in T:=\{(3),(5),(3,5),(3,5,3)\}$. By 3.4(1) and $2.3(1,2)$ we see that

(i) for distinct $c_{1}, c_{2} \in \Pi_{f}, c_{1}$ and $c_{2}$ are disjoint.

By 4.5(1), 
(ii) $3 \cdot\left|S_{(3,5,3)}\right|+\left|S_{(3)}\right|+3 \cdot\left|S_{(3,5)}\right|<4$.

By (i) and 2.2(1),

(iii) if $\left|S_{(3,5,3)}\right|+\left|S_{(3)}\right|+\left|S_{(3,5)}\right| \geqslant 1$, then $\left|S_{(5)}\right|=0$.

We may assume that the following holds:

(a) $\left|S_{(3,5,3)}\right|=0$. For otherwise, let $c \in S_{(3,5,3)}$. Note that $|P(c)|=4$. By 5.4(4b), $\operatorname{fr}_{f}(c)=\frac{2}{3}$. Hence, by (i) and (ii), $\operatorname{ch}^{*}(f) \leqslant 4-12+4 \cdot \frac{2}{3}+(12-4) \cdot \frac{1}{3}<0$.

(b) $\left|S_{(3,5)}\right|=0$. For otherwise let $c \in S_{(3,5)}$. Note that $|P(c)|=3$. By (i)-(iii) and (a), $\left|S_{(3,5)}\right|+\left|S_{(3)}\right|+\left|S_{(5)}\right|=1$. By $5.4(3 \mathrm{~b}), f_{f}(c)=\frac{8}{9}$. Hence, $\operatorname{ch}^{*}(f) \leqslant 4-12+3 \cdot \frac{8}{9}+$ $(12-3) \cdot \frac{1}{3}<0$.

Now, if $\left|S_{(5)}\right| \geqslant 1$, then by (iii), $\left|S_{(3)}\right|=0$. By 5.4(4b), if $c \in S_{(5)}$ then $\operatorname{fr}_{f}(c)=\frac{1}{2}$. Hence, $\operatorname{ch}^{*}(f) \leqslant 4-12+12 \cdot \frac{1}{2}<0$. If $\left|S_{(5)}\right|=0$, then by $4.5,\left|S_{(3)}\right| \leqslant 3$. By $5.4(1 \mathrm{~b})$, if $c \in S_{(3)}$ then $\operatorname{fr}_{f}(c) \leqslant \frac{3}{4}$. Hence, $\operatorname{ch}^{*}(f) \leqslant 4-12+6 \cdot \frac{3}{4}+(12-6) \cdot \frac{1}{3}<0$.

$|\boldsymbol{f}|=$ 11. By 2.3(0), $c$ does not extend $f$ by five, for every $c \in \Pi_{f}$. By 4.1,

$$
t(c) \in T:=\left\{(3),(5),(3,5),(3,5,3),\left(3,5^{3}\right),(6,3,6),(3,6),\left(6^{656} 6\right),\left(9,5^{3}, 3\right),(9,3,6)\right\}
$$

Let $T^{\prime \prime}:=\left\{(5),\left(6^{656} 6\right)\right\}$. By 5.4 , if $c \in \Pi_{f}$ and $c \in T^{\prime \prime}$, then $\operatorname{fr}\left(c^{\prime}, f\right) \leqslant \frac{1}{2}$. Also, by definition of $\Im_{F}, T \backslash T^{\prime \prime} \subseteq \Im_{F}$. We may assume that

(a) $\left|S_{(6,3,6)}\right|+\left|S_{\left(9,5^{3}, 3\right)}\right|+\left|S_{(9,3,6)}\right|=0$. For otherwise let

$$
T^{\prime}:=\left\{(3),(3,5),(3,5,3),\left(3,5^{3}\right),(6,3,6),(3,6)\right\}
$$

By 4.5, $\left|S_{(6,3,6)}\right|+\left|S_{\left(9,5^{3}, 3\right)}\right|+\left|S_{(9,3,6)}\right|=1$. Using 2.3(1), we conclude that $\left|S_{X}\right|=0$, for every $X \in T^{\prime}$. Let $c \in S_{(6,3,6)} \cup S_{\left(9,5^{3}, 3\right)} \cup S_{\left(9,5^{3}, 3\right)}(|P(c)|=4)$. By 5.4(8a,31,33), $\operatorname{fr}_{f}(c) \leqslant \frac{5}{6}$. Hence, $\operatorname{ch}^{*}(f) \leqslant 4-11+1 \cdot \frac{10}{3}+(11-4) \cdot \frac{1}{2}<0$.

(b) $\left|S_{(3,6)}\right|+\left|S_{\left(3,5^{3}\right)}\right|=0$. For otherwise let $T^{\prime}:=\{(3),(3,5),(3,5,3)\}$. By 2.3(0,1), we see that $\left|S_{(3,6)}\right|+\left|S_{\left(3,5^{3}\right)}\right|=1$, and $\left|S_{X}\right|=0$, for every $X \in T^{\prime}$. Let $c \in S_{(3,6)} \cup S_{\left(3,5^{3}\right)}$ $(|P(c)|=3)$. By $5.4(6 \mathrm{~b}, 5 \mathrm{~b}), \operatorname{fr}_{f}(c) \leqslant \frac{8}{9}$. Hence, $\mathrm{ch}^{*}(f) \leqslant 4-11+3 \cdot \frac{8}{9}+(11-3) \cdot \frac{1}{2}<0$.

(c) $\left|S_{(3,5,3)}\right|=0$. For otherwise let $T^{\prime}:=\{(3,5),(3,5,3)\}$. By 4.5(1), $\left|S_{\left(3,5^{3}\right)}\right|=1$, $\left|S_{(3)}\right| \leqslant 1$ and $\left|S_{X}\right|=0$, for every for $X \in T^{\prime}$. In addition, by $2.3(0,2),\left|S_{(5)}\right|=0$. Let $c \in S_{(3,5,3)}(|P(c)|=4)$. By 5.4 we see that $\operatorname{fr}_{f}(c)=\frac{2}{3}$, and if $c^{\prime} \in \Pi_{f}$ and $c^{\prime}=(3)$, then $\operatorname{fr}_{f}(c) \leqslant \frac{3}{4}$ to $f$. Hence, $\operatorname{ch}^{*}(f) \leqslant 4-11+4 \cdot \frac{2}{3}+2 \cdot \frac{3}{4}+(11-6) \cdot \frac{1}{2}<0$.

(d) $\left|S_{(3,5)}\right|=0$. For otherwise by $4.5(1),\left|S_{(3,5)}\right|=1$, and $\left|S_{(3)}\right| \leqslant 1$. Let $c \in S_{(3,5)}$ $(|P(c)|=3)$, and let $F(c)=\left\{f_{1}, f_{2}\right\}$ such that $\left|f_{1}\right|=3$ and $\left|f_{5}\right|=5$. By 2.3(0), $f_{1}$ is not adjacent to a 9 -face, and thus by $5.4(3 \mathrm{~b}), \mathrm{fr}_{f}(c)=\frac{7}{9}$. Hence, $\operatorname{ch}^{*}(f) \leqslant$ $4-11+3 \cdot \frac{7}{9}+2 \cdot \frac{3}{4}+(11-5) \cdot \frac{1}{2}<0$. 
Now, if $\left|S_{(5)}\right|+\left|S_{\left(6^{656} 6\right)}\right| \geqslant 1$, then $\left|S_{(3)}\right| \leqslant 1$, and $\mathrm{ch}^{*}(f) \leqslant 4-11+2 \cdot \frac{3}{4}+(11-2) \cdot \frac{1}{2} \leqslant 0$. If $\left|S_{(5)}\right|+\left|S_{\left(6^{656} 6\right)}\right|=0$, then, by $4.5\left|S_{(3)}\right| \leqslant 4$, and $\operatorname{ch}^{*}(f) \leqslant 4-11+8 \cdot \frac{3}{4}+(11-8) \cdot \frac{1}{3}=0$.

Suppose $|\boldsymbol{f}|=\mathbf{1 0}$. By $2.3(2)$ and $2.2(1), f$ is adjacent to at most one 5 -face. Also, $c$ does not extend $f$ by six, for every $c \in \Pi_{f}$. By 4.1 ,

$$
t(c) \in T:=\left\{(3),(5),(3,5),\left(3,5^{3}\right),(3,5,3),(6,3,6),(3,5,6,3),(3,6),\left(6^{6} 5^{6} 6\right),(3,6,5)\right\}
$$

We may assume that

(a) $\left|S_{(3,6,5)}\right|+\left|S_{(3,5,6,3)}\right|+\left|S_{\left(6^{6} 5^{6} 6\right)}\right|=0$. For otherwise, by 3.4(1), $\left|S_{(3,6,5)}\right|+\left|S_{(3,5,6,3)}\right|+$ $\left|S_{\left(6^{6} 5^{6} 6\right)}\right|=1$, and $\left|S_{X}\right|=0$, for every $X \in T \backslash\left\{(3,6,5),(3,5,6,3),\left(6^{6} 5^{6} 6\right)\right\}$. Let $c \in S_{(3,6,5)} \cup S_{(3,5,6,3)} \cup S_{\left(6^{6} 5^{6} 6\right)}(|P(c)| \leqslant 5)$. By 5.4(7,10a, 21), $\operatorname{fr}_{f}(c) \leqslant \frac{4}{5}$. Hence, $\operatorname{ch}^{*}(f) \leqslant 4-10+5 \cdot \frac{4}{5}+(10-5) \cdot \frac{1}{3}<0$.

(b) $\left|S_{(3,6)}\right|+\left|S_{(6,3,6)}\right|=0$. For otherwise, by $3.4(1),\left|S_{(3,6)}\right|+\left|S_{(6,3,6)}\right|=1,\left|S_{(3)}\right| \leqslant 1$, and $\left|S_{X}\right|=0$ for every $X \in \Im \backslash\{(3),(3,6),(6,3,6)\}$. Let $\{c\}=S_{(3,6)} \cup S_{(6,3,6)}$ $(|P(c)| \leqslant 4)$. By $5.4(1 \mathrm{c}, 6,8), \mathrm{fr}_{f}(c) \leqslant \frac{5}{6}$ and if $c^{\prime} \in \Pi_{f}$ with $t\left(c^{\prime}\right)=(3)$, then $\operatorname{fr}\left(c^{\prime}, f\right) \leqslant \frac{2}{3}$. Hence, $\operatorname{ch}^{*}(f) \leqslant 4-10+4 \cdot \frac{5}{6}+2 \cdot \frac{2}{3}+(10-6) \cdot \frac{1}{3}=0$.

(c) $\left|S_{\left(3,5^{3}\right)}\right|=0$. For otherwise, by $3.4(1),\left|S_{\left(3,5^{3}\right)}\right|=1,\left|S_{(3)}\right| \leqslant 1$, and $\left|S_{X}\right|=0$ for every $\left.X \in \Im \backslash\left\{(3), S_{\left(3,5^{3}\right.}\right)\right\}$. Let $c=S_{\left(3,5^{3}\right)}(|P(c)|=3)$. By $5.4, \operatorname{fr}_{f}(c)=\frac{8}{9}$. Hence, $\operatorname{ch}^{*}(f) \leqslant 4-10+3 \cdot \frac{8}{9}+2 \cdot \frac{2}{3}+(10-5) \cdot \frac{1}{3}<0$.

(d) $\left|S_{(3,5,3)}\right|=0$. For otherwise, by $3.4(1),\left|S_{(3,5,3)}\right|=1,\left|S_{(3)}\right| \leqslant 2$, and $\left|S_{X}\right|=0$ for every $X \in \Im \backslash\{(3),(3,5,3)\}$. Let $\{c\}=S_{(3,5,3)}(|P(c)|=4)$. By $5.4(4), \operatorname{fr}_{f}(c)=\frac{2}{3}$. Hence, $\operatorname{ch}^{*}(f) \leqslant 4-10+4 \cdot \frac{2}{3}+4 \cdot \frac{2}{3}+(10-8) \cdot \frac{1}{3}=0$.

(e) $\left|S_{(3,5)}\right|=0$. For otherwise, by $3.4(1),\left|S_{(3,5)}\right|=1,\left|S_{(3)}\right| \leqslant 2,\left|S_{X}\right|=0$ for every $X \in \Im \backslash\{(3),(3,5)\}$. Let $\{c\}=S_{(3,5)}$, and let $F(c)=\left\{f_{1}, f_{2}\right\}$ where $\left|f_{1}\right|=3$ and $\left|f_{2}\right|=5$. By $2.3(0), f_{1}$ is not adjacent to a 9 -face, and hence by $5.4(3 \mathrm{~b}), f_{f}(c)=\frac{7}{9}$. As $|P(c)|=3$, then $\mathrm{ch}^{*}(f) \leqslant 4-10+3 \cdot \frac{7}{9}+4 \cdot \frac{2}{3}+(10-7) \cdot \frac{1}{3}=0$.

(f) $\left|S_{(5)}\right|=0$. For otherwise, by $4.5(2),\left|S_{(5)}\right|=1,\left|S_{(3)}\right| \leqslant 2$ and $\left|S_{\left(6^{6} 5^{6} 6\right)}\right|=0$. Let $\{c\}=S_{(5)}(|P(c)|=2)$. By 5.4(2), $\operatorname{fr}_{f}(c)=\frac{1}{2}$. Hence, $\operatorname{ch}^{*}(f) \leqslant 4-10+2 \cdot \frac{1}{2}+4$. $\frac{2}{3}+(10-6) \cdot \frac{1}{3}<0$.

It follows, that if $c \in \Pi_{f}$, then $t(c)=(3)$. By 4.5, $\left|S_{(3)}\right| \leqslant 5$. Note that by $5.4(1 \mathrm{c})$, if $v \in V(f)$ then $v$ sends to $f$ a charge of at least $\frac{3}{5}$ at most $\frac{2}{3}$. If $\left|S_{(3)}\right| \leqslant 4$, then $\operatorname{ch}^{*}(f) \leqslant 4-10+8 \cdot \frac{2}{3}+(10-8) \cdot \frac{1}{3} \leqslant 0$. If $\left|S_{(3)}\right|=5$, then by $2.3(0), f$ is not adjacent to a 10-face. By $5.4(1 \mathrm{c}), \operatorname{ch}_{f}(c)=\frac{6}{5}$. Hence, $\operatorname{ch}^{*}(f) \leqslant 4-10+5 \cdot \frac{6}{5}=0$.

Suppose $|\boldsymbol{f}|=\mathbf{9}$. $c$ does not extend $f$ by seven, for every $c \in \Pi_{f}$. By 4.1, together with $2.8,2.15$ and $2.2(1)$, we conclude that

$$
\begin{gathered}
t(c) \in T:=\left\{(3),(5),(3,5),\left(3,5^{3}\right),(3,5,3),(6,3,6),(3,5,6,3),(3,6),(3,6,6,3),\right. \\
\left.(3,6,5),\left(6^{3} 6\right)\right\}
\end{gathered}
$$

We may assume that 
(a) $\left|S_{\left(6^{3} 6\right)}\right|=0$. For otherwise, by 3.4(4) and 2.3(0), it follows that $\left|S_{X}\right|=0$, for every $X \in T \backslash\left\{(3),\left(6^{3} 6\right)\right\}$. By 5.4 , if $c \in \Pi_{f}$ and $t(c) \in\left\{(3),\left(6^{3} 6\right)\right\}$, then $\operatorname{fr}_{f}(c) \leqslant 5 / 9$. Hence, $\operatorname{ch}^{*}(f) \leqslant 4-9+9 \cdot \frac{5}{9} \leqslant 0$.

(b) $\left|S_{(3,6,6,3)}\right|=0$. For otherwise, by $4.5(2),\left|S_{(3,6,6,3)}\right|=1$, and $\left|S_{X}\right|=0$, for every $X \in T \backslash\{(3,6,6,3)\}$. Let $c \in S_{(3,6,6,3)}(|P(c)|=5)$. By $5.4(13), \operatorname{fr}_{f}(c)=\frac{3}{5}$. Hence, $\operatorname{ch}^{*}(f) \leqslant 4-9+5 \cdot \frac{3}{5}+(9-5) \cdot \frac{1}{3}<0$.

(c) $\left|S_{(3,6,5)}\right|+\left|S_{(3,5,6,3)}\right|=0$. For otherwise, by 4.5, $\left|S_{(3,6,5)}\right|+\left|S_{(3,5,6,3)}\right|=1,\left|S_{X}\right|=0$, for every $X \in T \backslash\{(3,6,5),(3,5,6,3),(3)\}$, and $S_{(3)} \leqslant 1$. Let $c \in S_{(3,6,5)} \cup S_{(3,5,6,3)}$ $(|P(c)| \leqslant 5)$. By $5.4(7,10), \operatorname{fr}_{f}(c) \leqslant \frac{2}{3}$. Hence, $\operatorname{ch}^{*}(f) \leqslant 4-9+5 \cdot \frac{2}{3}+2 \cdot \frac{1}{2}+(9-7) \cdot \frac{1}{3}=$ 0 .

(d) $\left|S_{\left(3,5^{3}\right)}\right|+\left|S_{(3,6)}\right|+\left|S_{(6,3,6)}\right|=0$. For otherwise, by $4.5(2),\left|S_{\left(3,5^{3}\right)}\right|+\left|S_{(3,6)}\right|+\left|S_{(6,3,6)}\right|=$ $1, S_{(3)} \leqslant 2$, and $\left|S_{X}\right|=0$, for every $X \in T \backslash\left\{\left(3,5^{3}\right),(3,6),(6,3,6),(3)\right\}$. Let $c \in S_{\left(3,5^{3}\right)} \cup S_{(3,6)} \cup S_{(6,3,6)}(|P(c)| \leqslant 4)$. By 5.4, $\operatorname{fr}_{f}(c) \leqslant \frac{2}{3}$, and if $c^{\prime} \in \Pi_{f}$ with $t\left(c^{\prime}\right)=(3)$ then $\operatorname{fr}\left(c^{\prime}, f\right)=\frac{1}{2}$. Hence, $\operatorname{ch}^{*}(f) \leqslant 4-9+4 \cdot \frac{2}{3}+4 \cdot \frac{1}{2}+(9-8) \cdot \frac{1}{3}=0$.

(e) $\left|S_{(3,5,3)}\right|=0$. For otherwise, we have that $1 \leqslant\left|S_{(3,5,3)}\right| \leqslant 2$. If $\left|S_{(3,5,3)}\right|=2$, then by $4.5(2),\left|S_{X}\right|=0$, for every $X \in T \backslash\{(3,5,3)\}$. Hence, $\operatorname{ch}(f) \leqslant 4-9+2 \cdot 4 \cdot \frac{7}{12}+1 \cdot \frac{1}{3} \leqslant$ 0 . If $\left|S_{(3,5,3)}\right|=1$, then if $\left|S_{(3,5)}\right|=1, \operatorname{ch}^{*}(f) \leqslant 4-9+4 \cdot \frac{7}{12}+3 \cdot \frac{2}{3}+(9-7) \cdot \frac{1}{3} \leqslant 0$, and if $\left|S_{(3,5)}\right|=0$, then $\operatorname{ch}^{*}(f) \leqslant 4-9+\frac{7}{3}+(5) \cdot \frac{1}{2}<0$.

(f) $\left|S_{(3,5)}\right|=0$. For otherwise, we have that $1 \leqslant\left|S_{(3,5)}\right| \leqslant 2$. If $\left|S_{(3,5)}\right|=2$, then $\left|S_{X}\right|=0$, for every $X \in T \backslash\{(3,5)\}$, and $\operatorname{ch}(f) \leqslant 4-9+2 \cdot 3 \cdot \frac{2}{3}+3 \cdot \frac{1}{3} \leqslant 0$. Suppose then that $\left|S_{(3,5)}\right|=1$. If $\left|S_{(5)}\right|=1$, then $\left|S_{X}\right|=0$, for every $X \in T \backslash\{(3,5),(5)\}$ and $\operatorname{ch}^{*}(f) \leqslant 4-9+3 \cdot \frac{2}{3}+2 \cdot \frac{1}{2}+(9-5) \cdot \frac{1}{3} \leqslant 0$. If $\left|S_{(5)}\right|=0$, then $\left|S_{(3)}\right| \leqslant 3$, and $\operatorname{ch}^{*}(f) \leqslant 4-9+2+1 \cdot 3=0$.

It follows that if $\left|S_{X}\right| \geqslant 1$, for some $X \in T$, then $X \in\{(3),(5)\}$. By 5.4(1a,2), $\operatorname{ch}(f) \leqslant 4-9+4 \cdot 1+1 \cdot \frac{1}{3}<0$.

It remains to show that $\operatorname{ch}^{*}(f) \leqslant 0$, when $17 \leqslant|f| \leqslant 71$. We start with the following observation.

5.7. If $\left|\Pi_{\Im_{P}}\right| \leqslant 1$, then $\operatorname{ch}^{*}(f) \leqslant 0$.

Proof. By the definition of $\Im_{P}$ and 5.4 we may assume the following:

(i) If there exists a cluster $c \in \Pi_{f}$ such that $t(c) \in \Im_{P}$, then every $v \in V(P(c))$ sends to $f$ a charge of at most $\frac{17}{18}$;

(ii) In the particular case that $c \in \Pi_{f}$ and $t(c) \in\left\{(3,6,6,3,6,6,3),\left(5^{3}, 3,9,5,9,5^{3}, 3\right)\right\}$ then every $v \in V(P(c))$ sends to $f$ a charge of at most $\frac{5}{6}$; and

(iii) If there exists a cluster $c \in \Pi_{f}$ such that $t(c) \in \Im_{\text {all }} \backslash \Im_{P}$, then every $v \in V(P(c))$ sends to $f$ a charge of at most $\frac{2}{3}$.

If $\left|\Pi_{f}\left(\Im_{P}\right)\right|=0$, then by (iii) and (5), if $v \in V(f)$, then $v$ sends to $f$ a charge of at most $\frac{2}{3}$. Hence, $\operatorname{ch}^{*}(f) \leqslant 4-|f|+\frac{2}{3}(|f|)$, and for $|f| \geqslant 17, \operatorname{ch}^{*}(f) \leqslant 0$. Suppose that $\left|\Pi_{f}\left(\Im_{P}\right)\right|=1$. If $t(c) \in\left\{(3,6,6,3,6,6,3),\left(5^{3}, 3,9,5,9,5^{3}, 3\right\}\right.$, then $|P(c)|=8$. By 
(i), (ii) and (5), $\operatorname{ch}^{*}(f) \leqslant 4-|f|+8 \cdot \frac{5}{6}+\frac{2}{3}\left(|f|-8\right.$ ), and for $f \geqslant 17, \operatorname{ch}^{*}(f) \leqslant 0$. If $t(c) \notin\left\{(3,6,6,3,6,6,3),\left(5^{3}, 3,9,5,9,5^{3}, 3\right\}\right.$, then $|P(c)| \leqslant 6$. By (i), (ii) and (5), $\operatorname{ch}^{*}(f) \leqslant 4-|f|+6 \cdot \frac{17}{18}+\frac{2}{3}(|f|-6)$, and for $f \geqslant 17, \operatorname{ch}^{*}(f) \leqslant 0$.

As $\Pi_{f}\left(\Im_{P}\right) \subseteq \Pi_{f}\left(\Im_{F}\right)$, by 5.7, $\left|\Pi_{\Im_{P}}\right|,\left|\Pi_{\Im_{F}}\right| \geqslant 2$, which we may assume henceforth.

For every $X \in \Im_{\text {all }}$ let

$$
S_{X}=\left\{c \in \Pi_{f}: t(c)=X\right\} .
$$

By $3.4(1)$,

$$
|f|=\left|V_{s}\right|+\sum_{c \in \Pi_{f}}|P(c)|=\left|V_{s}\right|+\sum_{X \in \Im_{\text {all }}}\left|S_{X}\right| \cdot|P(X)| .
$$

Using (11) and the definition of $\Im_{\text {all }}$, (12) can be written as follows.

$$
\begin{aligned}
|f|= & \left|V_{s}\right|+\sum_{X \in \Im_{\text {all }}}\left(\left|S_{X}\right| \cdot|P(X)|\right)=\left|V_{s}\right|+2 \cdot\left|S_{(3)}\right|+3 \cdot\left|S_{(3,5)}\right|+2 \cdot\left|S_{(5)}\right|+ \\
& 3 \cdot\left|S_{\left(3,5^{3}\right)}\right|+4 \cdot\left|S_{(3,5,3)}\right|+4 \cdot\left|S_{(6,3,6)}\right|+5 \cdot\left|S_{(6,3,6,5)}\right|+ \\
& 5 \cdot\left|S_{(3,6,6,3)}\right|+5 \cdot\left|S_{(3,5,6,3)}\right|+6 \cdot\left|S_{(6,3,6,6,3)}\right|+6 \cdot\left|S_{(6,3,6,5,3)}\right|+ \\
& 3 \cdot\left|S_{(3,6)}\right|+3 \cdot\left|S_{\left(6^{3} 6\right)}\right|+4 \cdot\left|S_{\left(6^{3} 6,5\right)}\right|+5 \cdot\left|S_{\left(6^{6} 6,5,3\right)}\right|+ \\
& 3 \cdot\left|S_{\left(6^{6} 6\right.}\right|+4 \cdot\left|S_{\left(6^{6} 5^{6} 6\right)}\right|+4 \cdot\left|S_{(3,6,5)}\right|+5 \cdot\left|S_{\left(6^{3} 6,6,3\right)}\right|+ \\
& 5 \cdot\left|S_{(9,3,5,3)}\right|+6 \cdot\left|S_{(3,9,3,5,3)}\right|+5 \cdot\left|S_{(3,9,3,5)}\right|+5 \cdot\left|S_{(3,9,3,5)}\right|+ \\
& 4 \cdot\left|S_{\left(9,3,5^{3}\right)}\right|+5 \cdot\left|S_{\left(3,9,3,5^{3}\right)}\right|+5 \cdot\left|S_{\left(5,9,3,5^{3}\right)}\right|+6 \cdot\left|S_{\left(5,3,9,3,5^{3}\right)}\right|+ \\
& 6 \cdot\left|S_{\left(5^{3}, 3,9,3,5^{3}\right)}\right|+4 \cdot\left|S_{\left(9,5^{3}, 3\right)}\right|+5 \cdot\left|S_{\left(3,9,5^{3}, 3\right)}\right|+8 \cdot\left|S_{(3,6,6,3,6,6,3)}\right|+ \\
& 3 \cdot\left|S_{\left(6^{3} 6\right)}\right|+6 \cdot\left|S_{(3,6,5,6,3)}\right|+4 \cdot\left|S_{(9,3,6)}\right|+5 \cdot\left|S_{(3,9,3,6)}\right|+ \\
& 8 \cdot\left|S_{\left(5^{3}, 3,9,5,9,3,5^{3}\right)}\right|
\end{aligned}
$$

For $\Im \in\left\{\Im_{P}, \Im_{F}\right\}$ let

$$
\Pi_{f}(\Im)=\left\{c \in \Pi_{f}: t(c) \in \Im\right\} .
$$

By 4.5(1), if $|f| \geqslant 9$ and $\left|\Pi_{f}\left(\Im_{P}\right)\right| \geqslant 2$, then

$$
\sum_{X \in \Im_{P}}\left|S_{X}\right| \cdot M_{X}<\alpha(|f|)-|f|-\beta\left(|f|,\left|S_{\left(6^{6} 6\right)}\right|,\left|S_{\left(6^{6} 6,5\right)}\right|,\left|S_{\left(6^{3} 6\right)}^{9}\right|\right)
$$

By 4.5(2), if $k \in\{17,18\}$ and $\left|\Pi_{f}\left(\Im_{F}\right)\right| \geqslant 2$, then

$$
\sum_{X \in \Im_{F}}\left|S_{X}\right| \cdot M_{X}<\alpha(|f|)-|f|-\beta\left(|f|,\left|S_{\left(6^{6} 6\right)}\right|,\left|S_{\left(6^{6} 6,5\right)}\right|,\left|S_{\left(6^{3} 6\right)}^{9}\right|\right)
$$

We wish to write (15) and (16) in terms of the variables, $\left\{S_{X}\right\}_{X \in \Im_{\text {all }}}$. By the definition of $\Im_{P},(15)$ can be written as follows. 


$$
\begin{aligned}
& 1 \cdot\left|S_{(3)}\right|+3 \cdot\left|S_{(3,5)}\right|+4 \cdot\left|S_{\left(3,5^{3}\right)}\right|+3 \cdot\left|S_{(3,5,3)}\right|+4 \cdot\left|S_{(6,3,6)}\right|+ \\
& 7 \cdot\left|S_{(6,3,6,5)}\right|+6 \cdot\left|S_{(3,6,6,3)}\right|+5 \cdot\left|S_{(3,5,6,3)}\right|+8 \cdot\left|S_{(6,3,6,6,3)}\right|+ \\
& 7 \cdot\left|S_{(6,3,6,5,3)}\right|+8 \cdot\left|S_{\left(6^{3} 6,5,3\right)}\right|+9 \cdot\left|S_{\left(6^{3} 6,6,3\right)}\right|+8 \cdot\left|S_{(9,3,5,3)}\right|+ \\
& 8 \cdot\left|S_{(3,9,3,5,3)}\right|+7 \cdot\left|S_{(3,9,3,5)}\right|+7 \cdot\left|S_{(5,3,9,3,5)}\right|+8 \cdot\left|S_{\left(9,3,5^{3}\right)}\right|+8 \cdot\left|S_{\left(3,9,3,5^{3}\right)}\right|+ \\
& 7 \cdot\left|S_{\left(5,9,3,5^{3}\right)}\right|+8 \cdot\left|S_{\left(5,3,9,3,5^{3}\right)}\right|+9 \cdot\left|S_{\left(5^{3}, 3,9,3,5^{3}\right)}\right|+8 \cdot\left|S_{\left(9,5^{3}, 3\right)}\right|+9 \cdot\left|S_{\left(3,9,5^{3}, 3\right)}\right|+ \\
& 10 \cdot\left|S_{(3,6,6,3,6,6,3)}\right|+8 \cdot\left|S_{(3,6,5,6,3)}\right|+7 \cdot\left|S_{(9,3,6)}\right|+7 \cdot\left|S_{(3,9,3,6)}\right|+ \\
& 14 \cdot\left|S_{\left(5^{3}, 3,9,5,9,3,5^{3}\right)}\right|<\alpha(|f|)-|f|-\beta\left(|f|,\left|S_{\left(6^{6} 6\right)}\right|,\left|S_{\left(6^{3} 6,5\right)}\right|,\left|S_{\left(6^{3} 6\right)}\right|\right)
\end{aligned}
$$

By the definition of $\Im_{F}$, (16) can be written as follows:

$$
\begin{aligned}
& 3 \cdot\left|S_{(3,6)}\right|+4 \cdot\left|S_{(3,6,5)}\right|+ \\
& 1 \cdot\left|S_{(3)}\right|+3 \cdot\left|S_{(3,5)}\right|+4 \cdot\left|S_{\left(3,5^{3}\right)}\right|+3 \cdot\left|S_{(3,5,3)}\right|+4 \cdot\left|S_{(6,3,6)}\right|+ \\
& 7 \cdot\left|S_{(6,3,6,5)}\right|+6 \cdot\left|S_{(3,6,6,3)}\right|+5 \cdot\left|S_{(3,5,6,3)}\right|+8 \cdot\left|S_{(6,3,6,6,3)}\right|+7 \cdot\left|S_{(6,3,6,5,3)}\right|+ \\
& 8 \cdot\left|S_{\left(6^{3} 6,5,3\right)}\right|+9 \cdot\left|S_{\left(6^{3} 6,6,3\right)}\right|+8 \cdot\left|S_{(9,3,5,3)}\right|+8 \cdot\left|S_{(3,9,3,5,3)}\right|+7 \cdot\left|S_{(3,9,3,5)}\right|+ \\
& 7 \cdot\left|S_{(5,3,9,3,5)}\right|+8 \cdot\left|S_{\left(9,3,5^{3}\right)}\right|+8 \cdot\left|S_{\left(3,9,3,5^{3}\right)}\right|+7 \cdot\left|S_{\left(5,9,3,5^{3}\right)}\right|+8 \cdot\left|S_{\left(5,3,9,3,5^{3}\right)}\right|+ \\
& 9 \cdot\left|S_{\left(5^{3}, 3,9,3,5^{3}\right)}\right|+8 \cdot\left|S_{\left(9,5^{3}, 3\right)}\right|+9 \cdot\left|S_{\left(3,9,5^{3}, 3\right)}\right|+10 \cdot\left|S_{(3,6,6,3,6,6,3)}\right|+ \\
& 7 \cdot\left|S_{(9,3,6)}\right|+7 \cdot\left|S_{(3,9,3,6)}\right|+8 \cdot\left|S_{(3,6,5,6,3)}\right|+ \\
& 14 \cdot\left|S_{\left(5^{3}, 3,9,5,9,3,5^{3}\right)}\right|<\alpha(f)-|f|-\beta\left(|f|,\left|S_{\left(6^{3} 6\right)}\right|,\left|S_{\left(6^{3} 6,5\right)}\right|,\left|S_{\left(6^{3} 6\right)}\right|\right)
\end{aligned}
$$

Next the proof continues according to the following sketch. For each value of $|f|, 17 \leqslant$ $|f| \leqslant 71$, an integer program (IP henceforth) of the following form is constructed.

$$
\operatorname{maximize}(\operatorname{total}(f))
$$

subject to:

1. (13), and

2. (18) if $|f| \in\{17,18\}$, or (17) if $19 \leqslant|f| \leqslant 71$, and

3. Additional constraints based on the exact value of $|f|$.

For each value of $|f|$ from 17 to 71 , it will be shown that the maximum value of the expression in (19) is at most $|f|-4$. Hence by $(9), \operatorname{ch}^{*}(f) \leqslant 0$.

The first step is to derive an upper bound for total $(f)$ in term of the variables $\left\{S_{X}\right\}_{X \in \Im_{\text {all }}}$.

Let $c \in \Pi_{f}$. If $t(c) \in \Im_{\text {all }} \backslash\{(3),(3,5)\}$, then by $5.4, \operatorname{ch}_{f}(c)$ attains a unique value when $17 \leqslant|f| \leqslant 71$. If $t(c) \in\{(3),(3,5)\}$, then $\operatorname{ch}_{f}(c)$ attains several values, depending on the faces adjacent to the faces of $F(c)$. Using $5.4(1,2), S_{(3)}$ and $S_{(3,5)}$ are partitioned as follows:

Let $S_{(3)}^{3 / 2}, S_{(3)}^{7 / 5}, S_{(3)}^{4 / 3} \subseteq S_{(3)}$ be a partition of $S_{(3)}$ into three subsets, sending a charge of $\frac{3}{2}, \frac{7}{5}$ and $\frac{4}{3}$ to $f$, respectively. Let $S_{(3,5)}^{8 / 3}, S_{(3,5)}^{7 / 3} \subseteq S_{(3,5)}$ be a partition of $S_{(3,5)}$, into two subsets, sending a charge of $\frac{8}{3}$ and $\frac{7}{3}$ to $f$, respectively. By definition 


$$
\begin{gathered}
\left|S_{(3)}\right|=\left|S_{(3)}^{3 / 2}\right|+\left|S_{(3)}^{7 / 5}\right|+\left|S_{(3)}^{4 / 3}\right| . \\
\left|S_{(3,5)}\right|=\left|S_{(3,5)}^{8 / 3}\right|+\left|S_{(3,5)}^{7 / 3}\right| .
\end{gathered}
$$

The following easily follows from the definition of $\Pi_{f}, 5.4(1,2)$ and $2.2(1)$.

5.8. 1. Suppose $c \in S_{(3)}^{3 / 2} \cup S_{(3,5)}^{8 / 3}$. Then, there exists a 9-face $g$ such that $g$ is adjacent to $f$ and $g \notin F\left(c^{\prime}\right)$ for every $c^{\prime} \in \Pi_{f}$.

2. Suppose $\left|S_{(3,5)}^{8 / 3}\right| \geqslant 2$ and let $c_{1}, c_{2} \in S_{(3,5)}^{8 / 3}$ be distinct. Let $g_{1}, g_{2} \in F(G)$ such that for $i=1,2,\left|V\left(g_{i}\right)\right|=9, V\left(g_{i}\right) \cap V\left(c_{i}\right) \neq \emptyset$, and $c_{i}^{\prime}=g_{i} \cup c_{i}$ is a cluster of $f$ of type $(9,3,5)$. Then, $c_{1}$ and $c_{2}$ are disjoint.

Using 5.4, (20), (21), we obtain the following upper bound for total $(f)$.

$$
\begin{aligned}
& \operatorname{total}(f)=\sum_{c \in \Pi_{f}} \operatorname{ch}_{f}(c)+\frac{1}{3} \cdot\left|V_{s}\right| \leqslant \frac{1}{3}\left|V_{s}\right|+\frac{3}{2} \cdot\left|S_{(3)}^{3 / 2}\right|+\frac{7}{5} \cdot\left|S_{(3)}^{7 / 5}\right|+ \\
& \frac{4}{3} \cdot\left|S_{(3)}^{4 / 3}\right|+\frac{8}{3} \cdot\left|S_{(3,5)}^{8 / 3}\right|+\frac{7}{3} \cdot\left|S_{(3,5)}^{7 / 3}\right|+1 \cdot\left|S_{(5)}\right|+\frac{8}{3} \cdot\left|S_{\left(3,5^{3}\right)}\right|+\frac{8}{3} \cdot\left|S_{(3,5,3)}\right|+ \\
& \frac{10}{3} \cdot\left|S_{(6,3,6)}\right|+4 \cdot\left|S_{(6,3,6,5)}\right|+\frac{11}{3} \cdot\left|S_{(3,6,6,3)}\right|+4 \cdot\left|S_{(3,5,6,3)}\right|+5 \cdot\left|S_{(6,3,6,6,3)}\right|+ \\
& \frac{16}{3} \cdot\left|S_{(6,3,6,5,3)}\right|+2 \cdot\left|S_{(3,6)}\right|+2 \cdot\left|S_{\left(6^{3} 6\right)}\right|+\frac{8}{3} \cdot\left|S_{\left(6^{3} 6,5\right)}\right|+4 \cdot\left|S_{\left(6^{3} 6,5,3\right)}\right|+ \\
& \frac{7}{6} \cdot\left|S_{\left(66^{6} 666\right)}\right|+2 \cdot\left|S_{\left(6^{6} 5^{6} 6\right)}\right|+\frac{8}{3} \cdot\left|S_{(3,6,5)}\right|+\frac{11}{3} \cdot\left|S_{\left(6^{3} 6,6,3\right)}\right|+3 \frac{2}{3} \cdot\left|S_{(9,3,5,3)}\right|+ \\
& \frac{29}{6} \cdot\left|S_{(3,9,3,5,3)}\right|+\frac{25}{6} \cdot\left|S_{(3,9,3,5)}\right|+\frac{16}{3} \cdot\left|S_{(5,3,9,3,5)}\right|+\frac{19}{6} \cdot\left|S_{\left(9,3,5^{3}\right)}\right|+ \\
& \frac{13}{3} \cdot\left|S_{\left(3,9,3,5^{3}\right)}\right|+\frac{23}{6} \cdot\left|S_{\left(5,9,3,5^{3}\right)}\right|+\frac{11}{2} \cdot\left|S_{\left(5,3,9,3,5^{3}\right)}\right|+\frac{17}{3} \cdot\left|S_{\left(5^{3}, 3,9,3,5^{3}\right)}\right|+ \\
& 3 \cdot\left|S_{\left(9,5^{3}, 3\right)}\right|+\frac{25}{6} \cdot\left|S_{\left(3,9,5^{3}, 3\right)}\right|+\frac{20}{3} \cdot\left|S_{(3,6,6,3,6,6,3)}\right|+\frac{5}{3} \cdot\left|S_{\left(6^{3} 6\right)}\right|+ \\
& \frac{13}{3} \cdot\left|S_{(3,6,5,6,3)}\right|+\frac{8}{3} \cdot\left|S_{(9,3,6)}\right|+\frac{23}{6} \cdot\left|S_{(3,9,3,6)}\right|+\frac{20}{3} \cdot\left|S_{\left(5^{3}, 3,9,5,9,3,5^{3}\right)}\right|
\end{aligned}
$$

In some parts of the proof that follows, a computer was used for solving certain IPs. All IPs were solved by the second author by a simple $\mathrm{C}$ program that maximizes the objective function through a simple brute-force search over all possible values of the set of variables, and checked by the first author by using Maple's LPSolve function.

Suppose $|\boldsymbol{f}| \in\{\mathbf{2 0}, \ldots, \mathbf{7 1}\}$. The following IP is constructed.

$$
\begin{gathered}
\text { maximize }(\operatorname{total}(f)) \\
\text { subject to: }(13),(17),(20) \text { and }(21) .
\end{gathered}
$$


By solving it, we obtain that $\operatorname{total}(f) \leqslant|f|-4$ for each $|f| \in\{20, \ldots, 71\}$.

Suppose $|\boldsymbol{f}|=\mathbf{1 9}$. The proof follows by solving an IP which is identical to the one constructed above but with the following additional constraint:

$$
\left|S_{(3,5)}^{8 / 3}\right| \leqslant 1
$$

For the correctness of (23), suppose to the contrary that $\left|S_{(3,5)}^{8 / 3}\right| \geqslant 2$, and let $c_{1}, c_{2} \in S_{(3,5)}^{8 / 3}$ be distinct. By 5.8(2), $c_{1}$ and $c_{2}$ are disjoint; but then $f \cup c_{1} \cup c_{2} \subseteq G$ contains a 32-cycle; a contradiction.

Suppose $|\boldsymbol{f}| \in\{\mathbf{1 7}, \mathbf{1 8}\}$. For these two values, maximizing total $(f)$ is done by solving a sequence of IPs based on the value of $\left|S_{(3)}^{3 / 2}\right|+\left|S_{(3,5)}^{8 / 3}\right|$.

Case 1 Suppose

$$
\left|S_{(3)}^{3 / 2}\right|+\left|S_{(3,5)}^{8 / 3}\right|=0
$$

We split this into two cases.

Case 1.1 Suppose

$$
\left|S_{(6,3,6)}\right| \leqslant 1
$$

The proof follows by solving the following IP.

$$
\operatorname{maximize}(\operatorname{total}(f)) \text {. }
$$

subject to: (13), (18), (20), (21), (24) and (25).

\section{Case 1.2 Suppose}

$$
\left|S_{(6,3,6)}\right| \geqslant 2
$$

It will be shown below that

$$
\left|S_{(3)}^{7 / 5}\right|=0
$$

Then, the proof follows by solving the following IP.

$$
\operatorname{maximize}(\operatorname{total}(f)) \text {. }
$$

subject to: (13), (18), (20), (21), (24), (26), (27).

Correctness of (27) is verified as follows. Let $c_{1}, c_{2} \in S_{(6,3,6)}$ be distinct. Suppose for a contradiction that $\left|S_{(3)}^{7 / 5}\right| \geqslant 1$ and let $c_{3} \in S_{(3)}^{7 / 5}$. By 3.4(2), $c_{1}$ and $c_{2}$ are disjoint. By definition of $S_{(3)}^{7 / 5}$, there exists a 10-face, $f_{1}$, such that $c_{3}^{\prime}=f_{1} \cup c_{3}$, is a cluster of $f$ of type $(3,10)$. By 3.14(2), $c_{i}$ and $c_{3}^{\prime}$ are disjoint, for $i=1,2$. But then $c_{1} \cup c_{2} \cup c_{3}^{\prime} \subseteq G$ contains a 32-cycle; a contradiction.

Case 2 Suppose

$$
\left|S_{(3)}^{3 / 2}\right|+\left|S_{(3,5)}^{8 / 3}\right| \geqslant 1
$$

Case 2.1 Suppose first that

$$
\left|S_{(3,5)}^{8 / 3}\right|=0
$$


Consider $S_{(3)}^{3 / 2}$. By definition, if $c \in S_{(3)}^{3 / 2}$, then there exists a 9-face adjacent to $f$ and to the 3 -face of $F(c)$. Define $A$ to be the set of all such 9 -faces, i.e.,

$$
A=\left\{g \in \Gamma(f):|g|=9 \text { and there exists } c \in S_{(3)}^{3 / 2} \text { such that } V(g) \cap V(c) \neq \emptyset\right\}
$$

By 2.2(3), 2.3(1,5), and as $|f| \in\{17,18\}$ and $G$ contains no 32-cycles, it follows that $|A| \leqslant 2$. Hence,

$$
\left|S_{(3)}^{3 / 2}\right| \leqslant 4
$$

The rest is a case analysis on $\left|S_{(3)}^{3 / 2}\right|$.

Case 2.1.1 Suppose

$$
3 \leqslant\left|S_{(3)}^{3 / 2}\right| \leqslant 4
$$

In this case $|A|=2$. Hence, there exist distinct clusters $c_{1}$ and $c_{2}$ such that $t\left(c_{1}\right)=(3,9,3)$ and $t\left(c_{2}\right) \in\{(3,9),(3,9,3)\}$. By 2.3(1,5), $c_{1}$ and $c_{2}$ are disjoint. Observe that if $g$ is a 3-face adjacent to $f$, then $g \in F\left(c_{1}\right)$ or $g \in F\left(c_{2}\right)$, for otherwise $G$ contains a 32-cycle. By 3.14(1) and 2.3(0), it follows that

$$
\left|S_{\left(3,5^{3}\right)}\right|,\left|S_{(6,3,6)}\right|,\left|S_{(6,3,6,5)}\right|=0
$$

Next it is seen that

$$
\left|S_{(3)}^{7 / 5}\right|=0
$$

For suppose that $\left|S_{(3)}^{7 / 5}\right| \geqslant 1$. Let $c \in S_{(3)}^{7 / 5}, F(c)=\{g\}$ and $g_{1}$ be the 10 -face adjacent to $f$ and $g$. By 2.3(6), $g \notin F\left(c_{1}\right) \cup F\left(c_{2}\right)$, and $g$ is not adjacent to any of the faces of $A$. It follows that $G\left[E(g) \cup E\left(c_{1}\right) \cup E\left(c_{2}\right)\right]$ contains a 32-cycle, a contradiction. Hence (33) holds.

By 5.8(1) and the same considerations as above, it follows that

$$
\left|S_{\left(3,9,3,5^{3}\right)}\right|,\left|S_{\left(5,3,9,3,5^{3}\right)}\right|,\left|S_{\left(5^{3}, 3,9,3,5^{3}\right)}\right|=0
$$

The proof follows by solving the following IP.

$$
\operatorname{maximize}(\operatorname{total}(f)) \text {. }
$$

subject to: (13), (18), (20), (21), (29), (31), (32), (33) and (34).

Case 2.1.2 Suppose that

$$
1 \leqslant\left|S_{(3)}^{3 / 2}\right| \leqslant 2
$$

Let $c \in S_{(3)}^{3 / 2}$. Let $F(c)=\{g\}$, and let $g_{1}$ be the 9-face adjacent to $f$ and $g$. Let $\hat{c}=g \cup f$ be a cluster of type $(3,9)$. It is seen that

$$
\left|S_{\left(3,9,3,5^{3}\right)}\right|,\left|S_{\left(5,3,9,3,5^{3}\right)}\right|,\left|S_{\left(5^{3}, 3,9,3,5^{3}\right)}\right|=0
$$

For suppose that at least one of the sets $S_{\left(3,9,3,5^{3}\right)}, S_{\left(5,3,9,3,5^{3}\right)}$ and $S_{\left(5^{3}, 3,9,3,5^{3}\right)}$ is of size at least one. Let $c \in S_{\left(3,9,3,5^{3}\right)} \cup S_{\left(5,3,9,3,5^{3}\right)} \cup S_{\left(5^{3}, 3,9,3,5^{3}\right)}$. Then $c$ contains a sub-cluster, $c^{\prime}$, of type $\left(3,9,3,5^{3}\right)$ (possibly $c=c^{\prime}$ ). By 3.14(1), $c$ and $c_{1}^{\prime}$ are disjoint. But then $f \cup \hat{c} \cup c$ contains a 32-cycle, a contradiction. Hence (36) holds.

Next the proof of this case continues by considering the value $\left|S_{\left(3,5^{3}\right)}\right|+\left|S_{(3,5,6,3)}\right|+$ $\left|S_{(6,3,6)}\right|$. 
Case 2.1.2.1 Suppose

$$
\left|S_{\left(3,5^{3}\right)}\right|+\left|S_{(3,5,6,3)}\right|+\left|S_{(6,3,6)}\right|=0
$$

The proof follows by solving the following IP.

$$
\text { maximize }(\operatorname{total}(f)) \text {. }
$$

subject to: (13), (18), (20), (21), (28), (29), (35), (36), and (37).

\section{Case 2.1.2.2 Suppose}

$$
\left|S_{\left(3,5^{3}\right)}\right|+\left|S_{(3,5,6,3)}\right|+\left|S_{(6,3,6)}\right| \geqslant 1
$$

By (35), 3.14(1) and as $G$ contains no 32-cycles, it follows that

$$
\left|S_{\left(3,5^{3}\right)}\right|+\left|S_{(3,5,6,3)}\right|+\left|S_{(6,3,6)}\right|=1
$$

Three cases are possible.

1. Suppose

$$
\left|S_{(6,3,6)}\right|=1
$$

The proof follows by solving the following IP.

$$
\operatorname{maximize}(\operatorname{total}(f)) \text {. }
$$

subject to: (13), (18), (20), (21), (28), (29), (35), (39), and (40).

\section{Suppose}

$$
\left|S_{(3,5,6,3)}\right|=1
$$

Let $c \in S_{(3,5,6,3)}$. By 3.14(1), $\hat{c}$ and $c$ are disjoint.

It is shown that

$$
\left|S_{(3)}^{7 / 5}\right|+\left|S_{(3,5)}\right| \leqslant 2
$$

Let $c_{1} \in S_{(3)}^{7 / 5} \cup S_{(3,5)}$. For the correctness of (42) it suffices to show that if $g \in F\left(c_{1}\right)$ and $|g|=3$, then $V(g) \cap V(\hat{c}), V(g) \cap V(c)=\emptyset$.

(a) Suppose $c_{1} \in S_{(3,5)}$. By 3.4(2), $c$ and $c_{1}$ are disjoint. Also, $\hat{c}$ and $c_{1}$ are disjoint for otherwise $\hat{c} \cup c_{1}$ is a cluster of $f$ of type $(3,9,3,5)$ containing $c_{1}$; a contradiction to the definition of $\Pi_{f}$.

(b) If $c_{1} \in S_{(3)}^{7 / 5}$, then by 3.4(2), $c$ and $c_{1}$ are disjoint, and by 2.3(6), $\hat{c}$ and $c_{1}$ are disjoint.

The proof follows by solving the following IP.

$$
\operatorname{maximize}(\operatorname{total}(f)) \text {. }
$$

subject to: (13), (18), (20), (21), (28), (29), (35), (39), (41), and (42).

\section{Suppose}

$$
\left|S_{\left(3,5^{3}\right)}\right|=1
$$

By the same arguments as in the proof of (42), it follows that

$$
\left|S_{(3)}^{7 / 5}\right|+\left|S_{(3,5)}\right| \leqslant 3
$$

The proof follows by solving the following IP. 
$\operatorname{maximize}(\operatorname{total}(f))$.

subject to: (13), (18), (20), (21), (28), (29), (35), (39), (43), and (44).

Case 2.2 Suppose

$$
\left|S_{(3,5)}^{8 / 3}\right| \geqslant 1
$$

By 5.8(2), and as $G$ contains no 32-cycles we have

$$
\left|S_{(3,5)}^{8 / 3}\right| \leqslant 2
$$

Two cases are considered. Either $\left|S_{(3,5)}^{8 / 3}\right|=2$ or $\left|S_{(3,5)}^{8 / 3}\right|=1$.

Case 2.2.1 Suppose

$$
\left|S_{(3,5)}^{8 / 3}\right|=2
$$

Let $c_{1}, c_{2} \in S_{(3,5)}^{8 / 3}$. By 5.8(2) and the definition of $S_{(3,5)}^{8 / 3}$, there exist disjoint clusters, $c_{1}^{\prime}$ and $c_{2}^{\prime}$, of type $(9,3,5)$ such that $F\left(c_{1}\right) \subseteq F\left(c_{1}^{\prime}\right)$ and $F\left(c_{2}\right) \subseteq F\left(c_{2}^{\prime}\right)$. Suppose that $g$ is a 3-face adjacent to $f$. By 1.3 and the definition of $\Pi_{f}, g$ and $c_{i}$ are disjoint, for $i=1,2$. As $\left|S_{(3,5)}^{8 / 3}\right|=2$, and $G$ contains no 32-cycles, it follows that $g \in F\left(c_{1}\right) \cup F\left(c_{2}\right)$. As by the definition of $\Pi_{f}$ a face belongs to at most one cluster, then the two 3 -faces in $F\left(c_{1}\right) \cup F\left(c_{2}\right)$ are the sole 3 -faces adjacent to $f$. In particular we have

$$
\begin{gathered}
\left|S_{(3)}^{3 / 2}\right|,\left|S_{(3)}^{7 / 5}\right|,\left|S_{(3)}^{4 / 3}\right|,\left|S_{(6,3,6)}\right|,\left|S_{\left(3,9,3,5^{3}\right)}\right|,\left|S_{\left(5,3,9,3,5^{3}\right)}\right| \\
\left|S_{\left(5^{3}, 3,9,3,5^{3}\right)}\right|,\left|S_{(3,5,6,3)}\right|,\left|S_{\left(3,5^{3}\right)}\right|=0
\end{gathered}
$$

The proof follows by solving the following IP.

$$
\operatorname{maximize}(\operatorname{total}(f)) \text {. }
$$

subject to: (13), (17), (20), (21), (28), (47), and (48).

Case 2.2.2 Suppose

$$
\left|S_{(3,5)}^{8 / 3}\right|=1
$$

Either $\left|S_{(3)}^{3 / 2}\right| \geqslant 1$ or $\left|S_{(3)}^{3 / 2}\right|=0$.

Case 2.2.2.1 Suppose

$$
\left|S_{(3)}^{3 / 2}\right| \geqslant 1
$$

By 2.3 and as $G$ contains no 32 -cycles, it is seen that

$$
1 \leqslant\left|S_{(3)}^{3 / 2}\right| \leqslant 2
$$

By similar arguments as in Case (2.2.1), it follows that

$$
\begin{gathered}
\left|S_{(6,3,6,5,3)}\right|,\left|S_{(3)}^{7 / 5}\right|,\left|S_{(3)}^{4 / 3}\right|,\left|S_{(6,3,6)}\right|,\left|S_{\left(3,9,3,5^{3}\right)}\right|,\left|S_{\left(5,3,9,3,5^{3}\right)}\right|, \\
\left|S_{\left(5^{3}, 3,9,3,5^{3}\right)}\right|,\left|S_{(3,5,6,3)}\right|,\left|S_{\left(3,5^{3}\right)}\right|=0
\end{gathered}
$$

The proof follows by solving the following IP.

$$
\operatorname{maximize}(\operatorname{total}(f)) \text {. }
$$

subject to: (13), (17), (20), (21), (28), (49), (51), and (52). 
Case 2.2.2.2 Suppose

$$
\left|S_{(3)}^{3 / 2}\right|=0
$$

By similar arguments as in the cases above, it follows that

$$
\begin{gathered}
\left|S_{\left(5^{3}, 3,9,3,5^{3}\right)}\right|=0,\left|S_{\left(5,3,9,3,5^{3}\right)}\right|=0,\left|S_{(6,3,6,5,3)}\right|+\left|S_{(3,5,6,3)}\right|+\left|S_{(6,3,6)}\right|+\left|S_{\left(3,5^{3}\right)}\right| \leqslant 1 \\
\left|S_{(6,3,6,5)}\right|+\left|S_{(3,5)}\right| \leqslant 1,\left|S_{(6,3,6,5,3)}\right|+\left|S_{(3,5)}\right| \leqslant 1
\end{gathered}
$$

If

$$
\left|S_{\left(3,5^{3}\right)}\right|=0
$$

the proof follows by solving the following IP.

$$
\text { maximize }(\operatorname{total}(f)) \text {. }
$$

subject to: (13), (17), (20), (21), (28), (49), (53), (54), (55).

If,

$$
\left|S_{\left(3,5^{3}\right)}\right| \geqslant 1
$$

It is seen that,

$$
\left|S_{\left(3,5^{3}\right)}\right| \leqslant 1,\left|S_{(3)}\right| \leqslant 3
$$

The proof follows by solving the following IP.

$$
\operatorname{maximize}(\operatorname{total}(f)) \text {. }
$$

subject to: (13), (17), (20), (21), (28), (49), (53), (57), (58).

\section{$6 \quad$ Further Research}

This paper is hopefully another step towards resolving the Erdős-Gyárfás Conjecture; it might also be seen as an indication of what properties a counterexample would have to have. The next step could involve weakening one of the conditions of 1.1 (and most likely raising the upper bound on $m$ ); here, the authors consider how difficult it would be in each case.

Planarity could conceivably be replaced with projective-planarity, because the discharging process would still be feasible. (The projective plane has a positive Euler characteristic. The torus and Klein bottle both have Euler characteristic zero; using discharging on either of the latter surfaces also would require finding a face whose final charge is negative. Using a discharging argument on a surface with a negative Euler characteristic is possible - it has even been done - but it requires much more accounting than even the torus and Klein bottle cases.) However, many of the arguments in Section 2 would become more complex, because a few pairs of edges would be allowed to cross. Planarity thus seems to be the least likely of the hypotheses to weaken.

Replacing 3-connectivity with 2-connectivity looks more promising; the 3-connectivity of an alleged minimal counterexample was used only in a few places in Section 2. Once again, some further analysis would be required to obtain the contradictions needed to prove the lemmas in that section. 
Allowing vertices of degree four will ruin the proof of several lemma as well, since the fact that "two faces with a common vertex also have a common edge" was used early on as well. Vertices of higher degree should not be much more of a problem because (1) it can be assumed that no two vertices of degree greater than three are adjacent, and (2) vertices with larger degrees have negative charges, and these negative charges can be sent to a configuration that complicates the proof. It seems likely that, instead of proving that certain configurations are impossible, that it can be proven that they are possible, but there must be one or more vertices of large degree nearby.

\section{References}

[1] Paul Erdős, Some old and new problems in various branches of combinatorics, Discrete Math., 165 (1997), 227-231.

[2] Klas Markström, Extremal graphs for some problems on cycles in graphs, Cong. Numer., 171 (2004), 2179-2192.

[3] Stephen E. Shauger, Results on the Erdős-Gyárfás conjecture in $K_{1, m}$-free graphs, Proc. 29th Southeastern Int. Conf. Combinatorics, Graph Theory, and Computing (2004), 61-65.

[4] Dale Daniel and Stephen E. Shauger, A result on the Erdős-Gyárfás conjecture in planar graphs, Proc. 29th Southeastern Int. Conf. Combinatorics, Graph Theory, and Computing (2001), 129-139. 


\section{Appendix: Properties of Clusters}

The following page summarizes most of the important information related to the clusters present in this paper. $\left(M_{c}=\max \Omega_{c}\right.$. $)$

\begin{tabular}{|c|c|c|c|c|c|}
\hline \multirow[b]{2}{*}{$t(c)$} & \multirow[b]{2}{*}{$|P(c)|$} & \multirow[b]{2}{*}{$c \in$} & \multirow[b]{2}{*}{$\Omega_{c}$} & \multicolumn{2}{|c|}{$\left(\operatorname{ch}_{f}(c), \operatorname{fr}_{f}(c)\right)$} \\
\hline & & & & $|f|=9$ & $|f|>9$ \\
\hline$(3)$ & 2 & $\Im_{S}, \Im_{F}, \Im_{P}$ & $\{0,1\}$ & $\left(1, \frac{1}{2}\right)$ & See 5.4 \\
\hline$(3,5)$ & 3 & $\Im_{S}, \Im_{F}, \Im_{P}$ & {$[0,3]$} & $\left(2, \frac{2}{3}\right)$ & See 5.4 \\
\hline$(5)$ & 2 & $\Im_{S}$ & $\{0,3\}$ & $\left(1, \frac{1}{2}\right)$ & $\left(1, \frac{1}{2}\right)$ \\
\hline$(3,5,3)$ & 4 & $\Im_{S}, \Im_{F}, \Im_{P}$ & {$[0,3]$} & $\left(\frac{7}{3}, \frac{7}{12}\right)$ & $\left(\frac{8}{3}, \frac{2}{3}\right)$ \\
\hline$(6,3,6)$ & 5 & $\Im_{S}, \Im_{F}, \Im_{P}$ & {$[0,4] \backslash 2$} & $\left(\frac{8}{3}, \frac{2}{3}\right)$ & $\left(\frac{10}{3}, \frac{5}{6}\right)$ \\
\hline$(6,3,6,5)$ & 5 & $\Im_{S}, \Im_{F}, \Im_{P}$ & {$[0,7] \backslash 2$} & - & $\left(4, \frac{4}{5}\right)$ \\
\hline$(3,6,6,3)$ & 5 & $\Im_{S}, \Im_{F}, \Im_{P}$ & {$[0,6] \backslash 2$} & $\left(3, \frac{3}{5}\right)$ & $\left(\frac{11}{3}, \frac{11}{5}\right)$ \\
\hline$(3,5,6,3)$ & 5 & $\Im_{S}, \Im_{F}, \Im_{P}$ & {$[0,5] \backslash 2$} & $\left(\frac{10}{3}, \frac{2}{3}\right)$ & $\left(4, \frac{4}{5}\right)$ \\
\hline$(6,3,6,6,3)$ & 6 & $\Im_{S}, \Im_{F}, \Im_{P}$ & {$[0,8] \backslash 5$} & - & $\left(5, \frac{5}{6}\right)$ \\
\hline$(6,3,6,5,3)$ & 6 & $\Im_{S}, \Im_{F}, \Im_{P}$ & {$[0,7] \backslash\{2,5\}$} & - & $\left(\frac{16}{3}, \frac{8}{9}\right)$ \\
\hline$(3,6)$ & 3 & $\Im_{S}, \Im_{F}$ & {$[0,4] \backslash 2$} & $\left(\frac{5}{3}, \frac{5}{9}\right)$ & $\left(2, \frac{2}{3}\right)$ \\
\hline$(3,6,5)$ & 4 & $\Im_{S}, \Im_{F}$ & {$[0,5] \backslash 2$} & $\left(\frac{7}{3}, \frac{7}{12}\right)$ & $\left(\frac{8}{3}, \frac{2}{3}\right)$ \\
\hline$(9,3,5,3)$ & 5 & $\Im_{S}, \Im^{9}, \Im_{F}, \Im_{P}$ & {$[0,8] \backslash 5$} & - & $\left(\frac{11}{3}, \frac{11}{15}\right)$ \\
\hline$(3,9,3,5,3)$ & 6 & $\Im_{S}, \Im^{9}, \Im_{F}, \Im_{P}$ & {$[0,8] \backslash 5$} & - & $\left(\frac{29}{6}, \frac{29}{36}\right)$ \\
\hline$(3,9,3,5)$ & 5 & $\Im_{S}, \Im^{9}, \Im_{F}, \Im_{P}$ & {$[0,7] \backslash\{2,5\}$} & - & $\left(\frac{25}{6}, \frac{5}{6}\right)$ \\
\hline$(5,3,9,3,5)$ & 6 & $\Im_{S}, \Im^{9}, \Im_{F}, \Im_{P}$ & {$[0,7] \backslash\{2,5\}$} & - & $\left(\frac{16}{3}, \frac{8}{9}\right)$ \\
\hline$(3,6,6,3,6,6,3)$ & 8 & $\Im_{S}, \Im_{F}, \Im_{P}$ & {$[0,10]$} & - & $\left(\frac{20}{3}, \frac{5}{6}\right)$ \\
\hline$(3,6,5,6,3)$ & 6 & $\Im_{S}, \Im_{F}, \Im_{P}$ & {$[0,8] \backslash 5$} & - & $\left(\frac{13}{3}, \frac{13}{18}\right)$ \\
\hline$(9,3,6)$ & 4 & $\Im_{S}, \Im^{9}, \Im_{F}, \Im_{P}$ & {$[0,7] \backslash\{2,5\}$} & - & $\left(\frac{8}{3}, \frac{2}{3}\right)$ \\
\hline$(3,9,3,6)$ & 5 & $\Im_{S}, \Im^{9}, \Im_{F}, \Im_{P}$ & {$[0,7] \backslash\{2,5\}$} & - & $\left(\frac{23}{6}, \frac{23}{30}\right)$ \\
\hline$\left(3,5^{3}\right)$ & 3 & $\Im_{C}, \Im_{C}^{3}, \Im_{F}, \Im_{P}$ & {$[0,4]$} & $\left(2, \frac{2}{3}\right)$ & $\left(\frac{8}{3}, \frac{8}{9}\right)$ \\
\hline$\left(6^{656} 6\right)$ & 3 & $\Im_{C}, \Im_{C}^{5}$ & $\{0\}$ & - & $\left(\frac{7}{6}, \frac{7}{18}\right)$ \\
\hline$\left(6^{6} 5^{6} 6\right)$ & 4 & $\Im_{C}, \Im_{C}^{5}$ & $\{0\}$ & - & $\left(2, \frac{1}{2}\right)$ \\
\hline$\left(6^{3} 6\right)$ & 3 & $\Im_{C}, \Im_{C}^{6}$ & $\{0,6\}$ & $\left(2, \frac{2}{3}\right)$ & $\left(2, \frac{2}{3}\right)$ \\
\hline$\left(6^{3} 6,5\right)$ & 4 & $\Im_{C}, \Im_{C}^{6}$ & $\{0,6\}$ & $\left(\frac{8}{3}, \frac{2}{3}\right)$ & $\left(\frac{8}{3}, \frac{2}{3}\right)$ \\
\hline$\left(6^{3} 6,5,3\right)$ & 5 & $\Im_{C}, \Im_{C}^{6}, \Im_{F}, \Im_{P}$ & {$[0,8] \backslash 5$} & - & $\left(4, \frac{4}{5}\right)$ \\
\hline$\left(6^{3} 6,6,3\right)$ & 5 & $\Im_{C}, \Im_{C}^{6}, \Im_{F}, \Im_{P}$ & {$[0,9] \backslash 5$} & - & $\left(\frac{11}{3}, \frac{11}{15}\right)$ \\
\hline$\left(6^{3} 6\right)$ & 3 & $\Im_{C}, \Im_{C}^{6 *}$ & $\{0,6\}$ & $\left(\frac{5}{3}, \frac{5}{9}\right)$ & $\left(\frac{5}{3}, \frac{5}{9}\right)$ \\
\hline$\left(9,3,5^{3}\right)$ & 4 & $\Im_{C}, \Im_{C}^{9}, \Im^{9}, \Im_{F}, \Im_{P}$ & {$[0,8] \backslash 5$} & - & $\left(\frac{19}{6}, \frac{19}{24}\right)$ \\
\hline$\left(3,9,3,5^{3}\right)$ & 5 & $\Im_{C}, \Im_{C}^{9}, \Im^{9}, \Im_{F}, \Im_{P}$ & {$[0,8] \backslash 5$} & - & $\left(\frac{13}{3}, \frac{13}{15}\right)$ \\
\hline$\left(5,9,3,5^{3}\right)$ & 5 & $\Im_{C}, \Im_{C}^{9}, \Im^{9}, \Im_{F}, \Im_{P}$ & {$[0,7] \backslash\{2,5\}$} & - & $\left(\frac{23}{6}, \frac{23}{30}\right)$ \\
\hline$\left(5,3,9,3,5^{3}\right)$ & 6 & $\Im_{C}, \Im_{C}^{9}, \Im^{9}, \Im_{F}, \Im_{P}$ & {$[0,8] \backslash 5$} & - & $\left(\frac{11}{2}, \frac{11}{12}\right)$ \\
\hline$\left(5^{3}, 3,9,3,5^{3}\right)$ & 6 & $\Im_{C}, \Im_{C}^{9}, \Im^{9}, \Im_{F}, \Im_{P}$ & {$[0,9] \backslash 2$} & - & $\left(\frac{17}{3}, \frac{17}{18}\right)$ \\
\hline$\left(9,5^{3}, 3\right)$ & 4 & $\Im_{C}, \Im_{C}^{9}, \Im^{9}, \Im_{F}, \Im_{P}$ & {$[0,8] \backslash 5$} & - & $\left(3, \frac{3}{4}\right)$ \\
\hline$\left(3,9,5^{3}, 3\right)$ & 5 & $\Im_{C}, \Im_{C}^{9}, \Im^{9}, \Im_{F}, \Im_{P}$ & {$[0,9] \backslash 2$} & - & $\left(\frac{25}{6}, \frac{5}{6}\right)$ \\
\hline$\left(5^{3}, 3,9,5,9,3,5^{3}\right)$ & 4 & $\Im_{C}, \Im_{C}^{9}, \Im^{9}, \Im_{F}, \Im_{P}$ & {$[0,14]$} & - & $\left(\frac{20}{3}, \frac{5}{6}\right)$ \\
\hline
\end{tabular}

